THESIS

EVALUATING SATELLITE-BASED CLOUD PERSISTENCE AND DISPLACEMENT NOWCASTING TECHNIQUES OVER COMPLEX TERRAIN

\author{
Submitted by \\ Eric M. Guillot \\ Department of Atmospheric Science
}

In partial fulfillment of the requirements

For the Degree of Master of Science

Colorado State University

Fort Collins, $\mathrm{CO}$

Fall 2010 
COLORADO STATE UNIVERSITY

August 13, 2010

WE HEREBY RECOMMEND THAT THE THESIS PREPARED UNDER OUR SUPERVISION BY ERIC M. GUILLOT ENTITLED EVALUATING SATELLITEBASED CLOUD PERSISTENCE AND DISPLACEMENT NOWCASTING TECHNIQUES OVER COMPLEX TERRAIN BE ACCEPTED AS FULFILLING IN PART REQUIREMENTS FOR THE DEGREE OF MASTER OF SCIENCE.

Committee on Graduate Work

Colette L. Heald

Steven C. Reising

Advisor: Thomas H. Vonder Haar

Department Head: Richard H. Johnson 


\section{ABSTRACT OF THESIS \\ EVALUATING SATELLITE-BASED CLOUD PERSISTENCE AND DISPLACEMENT NOWCASTING TECHNIQUES OVER COMPLEX TERRAIN}

Cloud nowcasting (0-6 hour forecasts) is an important area of study for weather forecasting, solar energy estimation, and Department of Defense (DoD) applications. The DoD is developing data assimilation methods to predict cloud movement. However these systems require valuable time to run and are not always accurate. Because many military operations are of a time-sensitive nature, fast-processing cloud nowcasting techniques are required. Satellite imagery has shown that clouds move at varying speeds and in different directions, while some tend not to move at all. We test the hypothesis that clouds forced by complex terrain do not displace with the wind, but instead persist along the barrier on which they were formed. Thus, a combination of persistence and displacement techniques in regions of complex terrain are expected to yield a better forecast than either of them alone.

The Moderate Resolution Imaging Spectroradiometer (MODIS) sensor aboard both the Terra and Aqua satellites allows for the same region of Earth to be sampled twice by each satellite in a roughly 2-4 hour timeframe. This allows a 2-4 hour cloud nowcast to be created and tested. Using the MODIS Cloud Mask algorithm at $5 \mathrm{~km}$ resolution (interpolated to $1 \mathrm{~km}$ ) and wind data from local weather balloon soundings, a cloud climatology nowcast, a cloud persistence nowcast, a cloud 700mb wind nowcast, a 
cloud various wind speed nowcast, and a cloud terrain-influenced nowcast were developed from December 2008 through November 2009 over Utah and southwestern Wyoming. Persistence/displacement forecasts were also conducted based on the phase of the cloud, as determined by the MODIS Cloud Phase algorithm. A new forecast skill evaluation scheme was also introduced, designed to equally appreciate correct cloudy areas and correct clear areas.

Contrary to our hypothesis, the persistence nowcasting method demonstrated the best skill, especially in the winter months, by as much as $10 \%$ critical success index (CSI) over the other methods. The Persistence Method, 700mb Method, and Various Winds Method performed similarly during the summer months ( $65 \%$ CSI for all three).

Use of cloud phase information revealed that ice cloud persistence, while displacing either liquid-water clouds or mixed-phase clouds yielded the highest CSI scores, but the resulting scores were still lower than the Persistence Method. We conclude that cloud analysis at high resolution over complex terrain in Utah, using no model wind or moisture data, cannot improve upon a persistence nowcast over Utah. However, because these basic nowcasting methods can be run and their skill evaluated in less than two minutes, educated decisions can be made nearly instantaneously.

Eric M. Guillot Department of Atmospheric Science Colorado State University Fort Collins, CO 80523

Fall 2010 


\section{ACKNOWLEDGEMENTS}

This research was made possible through the overwhelming support of several individuals. I would first like to thank my advisor, Tom Vonder Haar, for giving me the opportunity to attend Colorado State University and suggesting to me a thesis topic that I thoroughly enjoyed. I would also like to thank my committee members, Colette Heald and Steven Reising, for their insight and input to make this thesis better. I would like to thank John Forsythe of CIRA for the countless hours he spent assisting me with the direction in which to take this research as well as taking the time to help with the day-today problems I encountered. I would also like to thank Steve Fetcher of CIRA for his mathematical insight regarding our skill score system.

I want to thank the Army Research Laboratory in White Sands, New Mexico for allowing me to visit and collaborate with them to further tailor my research topic to their specific needs. I would like to thank my office mates in room 301 for the past two years: Darren Van Cleave, Heather Cronk, Kelly Howell, and Jessica Ram. Their hours of help, insight, support, and distractions helped keep me both focused and positive as this research was conducted. I also offer thanks to my three roommates: Ghassan Alaka, David Henderson, and Jeremiah Sjoberg. Finally, I want to thank my family and friends for their love and support throughout this process.

This research was supported by the Department of Defense Center for Geosciences/Atmospheric Research at Colorado State University under Cooperative Agreement W911NF-06-2-0015 with the Army Research Laboratory. 


\section{TABLE OF CONTENTS}

Abstract of Thesis..................................................................

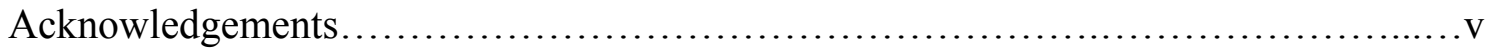

Table of Contents................................................................

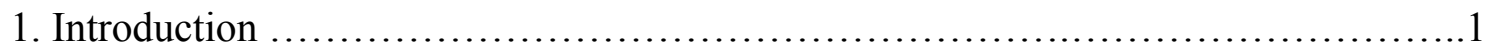

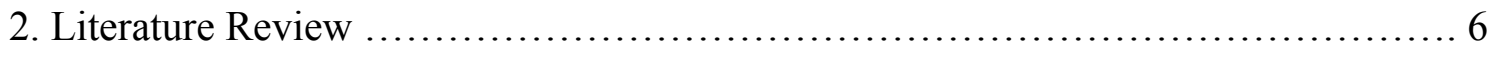

2.1 Persistence Cloud Nowcasting Methods......................................6

2.2 Displacement Cloud Nowcasting Methods................................ 9

2.3 Additional Cloud Nowcasting Methods..................................14

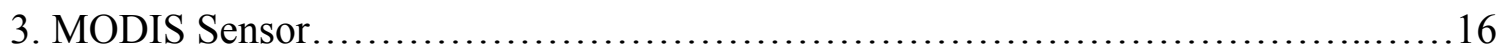

3.1 MODIS Cloud Mask Algorithm.......................................16

3.2 MODIS Cloud-Top Properties Algorithm..................................20

3.3 MODIS Cloud Phase Algorithm.......................................... 23

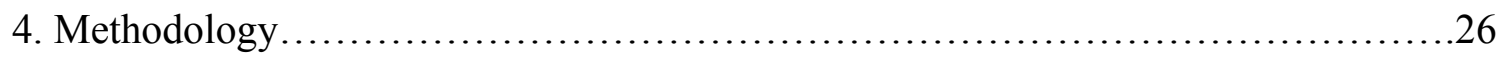

4.1 Spatial Domain Considerations.........................................26

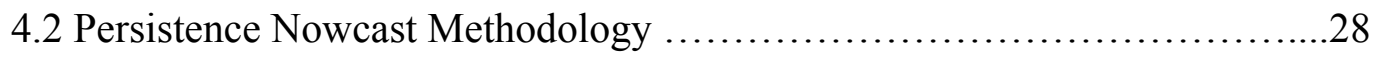

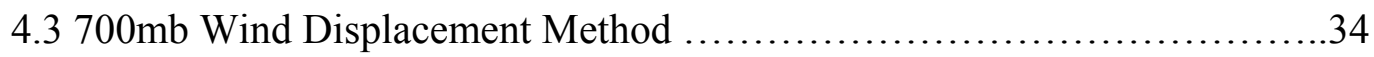

4.4 Various Height Wind Displacement Method...............................38

4.5 Terrain Wind Displacement Method.....................................41

4.6 Cloud Climatology Method...........................................43

4.7 Cloud Phase Displacement Method......................................44

4.8 Temporal Domain Considerations......................................45 
4.9 Forecast Skill Evaluation.

5. Results.

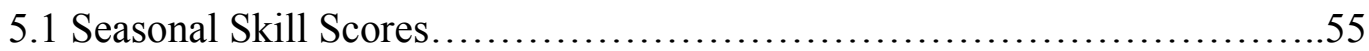

5.2 Seasonal Skill Score Variations........................................65

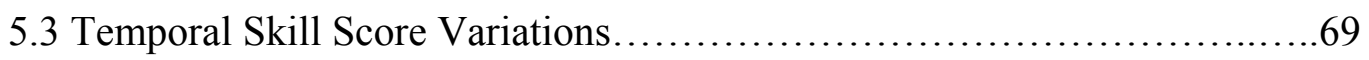

5.4 Skill Domain Distribution............................................. 71

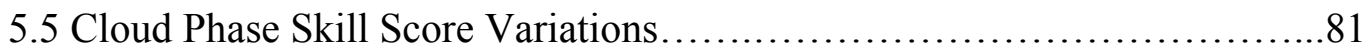

5.6 Synoptic Weather Contributions to Nowcasting Skill...................... 90

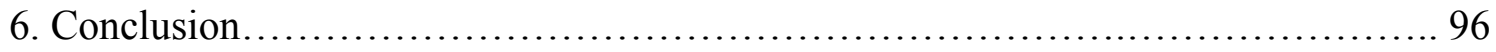

6.1 Research Conclusions.......................................................96

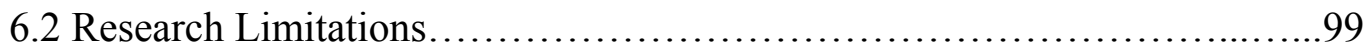

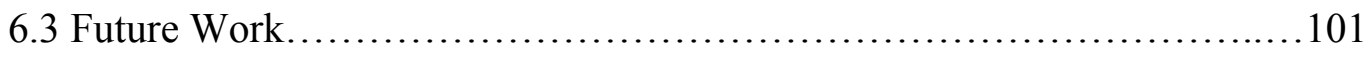

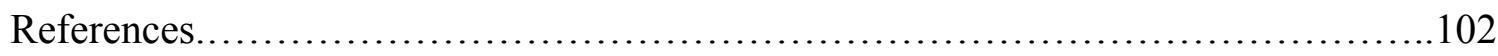




\section{INTRODUCTION}

Forecasting the presence and movement of clouds poses a significant problem for both civilian and military organizations. The weather forecaster is interested in correctly forecasting both precipitation location and surface temperature. The solar energy consultant needs to know the general trend of cloud movement to choose the most efficient place to set up a solar farm. The military's interest in cloud forecasting (particularly short-term forecasting) stems from their concern with all possible variables that could potentially affect the "battlespace," and ultimately, the mission at hand.

Unmanned Aerial Vehicles (UAVs) are often sent ahead of an approaching battle group in order to visually inspect the target area. These small planes are equipped with cameras to relay information vital to the success of the mission. The presence of clouds prevents these cameras from accurately photographing the area in question. More importantly, because these UAVs are so small relative to the size of a typical airplane (often no larger than a few meters), they are very prone to icing and turbulence (Sharman 2010). Thus, any clouds the aircraft may encounter on its flight path can severely cripple it or even cause it to crash. Such a mission failure coupled with the loss of expensive equipment has been deemed unacceptable by the United States military.

Satellite reconnaissance imaging is another military program that is dependent on correctly forecasting cloud cover over a given area. Department of Defense satellites in orbit are tasked with recording visual and/or infrared images over unfriendly territory, but cloud cover can make this impossible. Thus, forecasting the location of clouds over the region in question before the satellite begins to take photographs avoids a drain on 
resources as well as informs the operators that repositioning the satellite may be a prudent course of action.

Risk assessment is a third application for the military to use cloud short-term forecasting. The Tri-Service Information Weather Effects Decision Aid (T-IWEDA) is a software package designed to assimilate every possible variable that can affect a mission and assign a risk to various means of carrying out that mission (Shirkey 2010). For example, if a mission is deemed to have less risk if cloud cover is present, the quantitative results of short-term cloud forecasts would be input to T-IWEDA. Thus, the overall risk to the mission would increase if the cloud forecast yielded clear skies during the mission timeframe. Since the military bases most of their operations on overall risk, cloud forecasting is a necessary input.

Nowcasting is defined by the American Meteorological Society Glossary as "a short-term weather forecast, generally for the next few hours," (AMS Glossary, 2010). Specifically, "the U.S. National Weather Service specifies zero to three hours, though up to six hours may be used by some." This definition directly relates to the timeframe of interest to this thesis, and as such, "cloud nowcasting" will be used to replace "short-term cloud forecasting" herein.

In recent years, cloud nowcasting using models that include data assimilation has become increasingly popular. A model's ability to ingest radiance data directly from a satellite in orbit to produce clouds has much potential in the coming years. However, although modelers remain optimistic about data assimilation, it is far from operational use. Often (depending on other fields ingested in the model such as pressure and relative humidity), the model will omit a cloud because it decides that it is too dry to support a 
cloud, even though a cloud has been observed in that area. Conversely, clouds are often created by the model when no cloud has been observed. In addition to inaccurate cloudfield representations, models often simply take too long to ingest this data and output a result. In certain situations (especially in the military), a decision may need to be made quickly, without time to let the model come to a steady-state and output useful results. Time-sensitive decisions, especially when human life is at risk, sometimes cannot wait for computer model output.

This research addresses these problems by offering several fast cloud nowcasting methods that can be applied to any region of the world. Furthermore, this research offers a unique approach to the cloud nowcasting problem by using only observational data through a combination of both persistence and displacement of cloud nowcasting techniques in order to yield the most accurate cloud nowcast possible. It is our hypothesis that a combination of persistence and displacement cloud nowcasting techniques that takes into account local topography will exhibit more forecast skill (Figure 1) than a technique that utilizes only persistence or only displacement techniques. That is, a method that predicts cloud persistence over regions of higher terrain while displacing clouds that are not over elevated areas should exhibit more forecast skill than those that simply predict only persistence or only displacement of all clouds. This is due to the topographic influence on the overall cloud scheme, usually regenerating stationary clouds as upslope winds during the day allow thermals to rise to their lifting condensation level. 


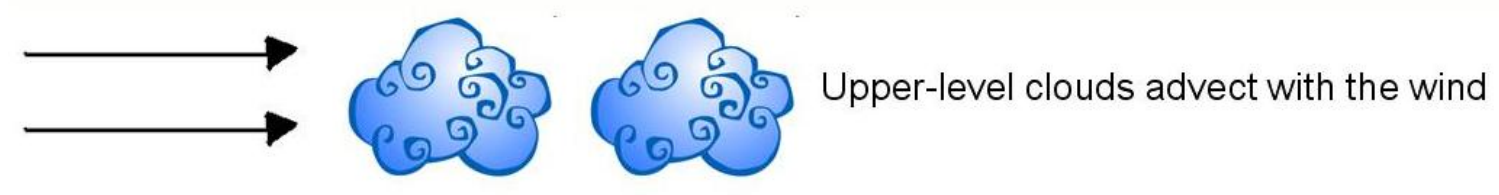
Low-level clouds continuously regenerate

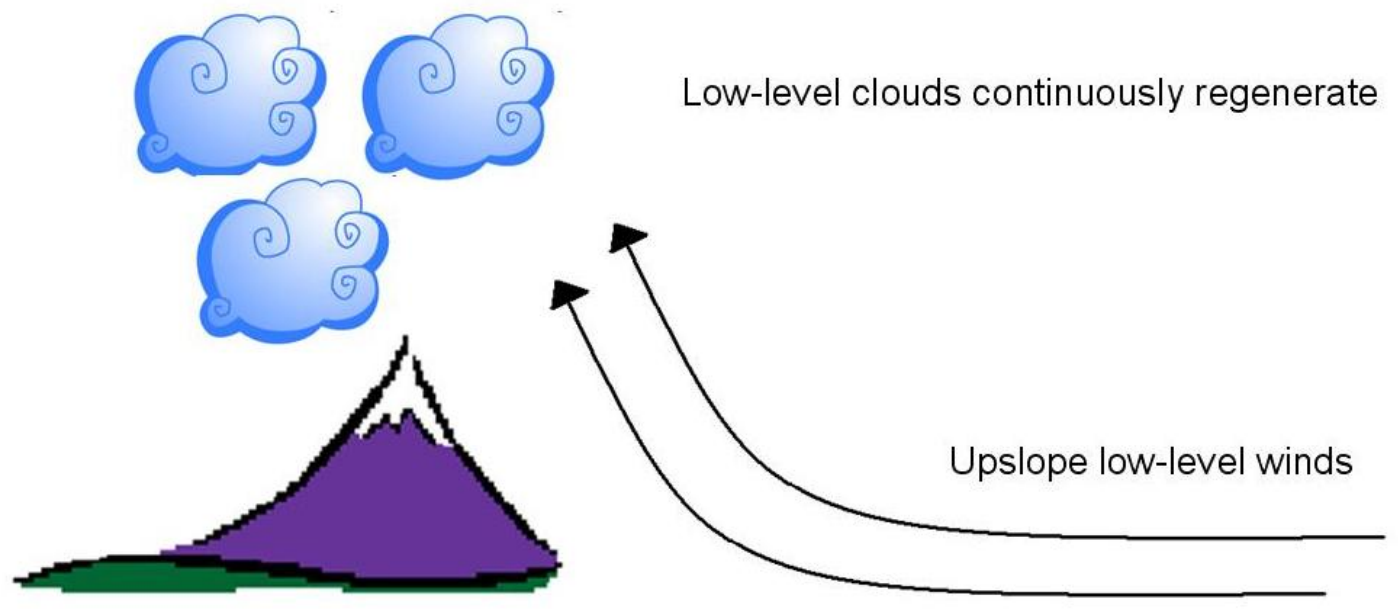

Fig 1: Illustration of our hypothesis that low-level clouds tend to persist over terrain, while upper-level clouds displace with the wind.

Figure (2) shows an idealized cloud forecast time series for various popular methods. This figure is not based on any empirical data. Rather, it is strictly conceptual in order to assist in visualizing the cloud forecasting problem. Note that theory suggests (in our nowcasting timeframe) cloud persistence techniques should perform better than cloud advection (displacement) techniques. 


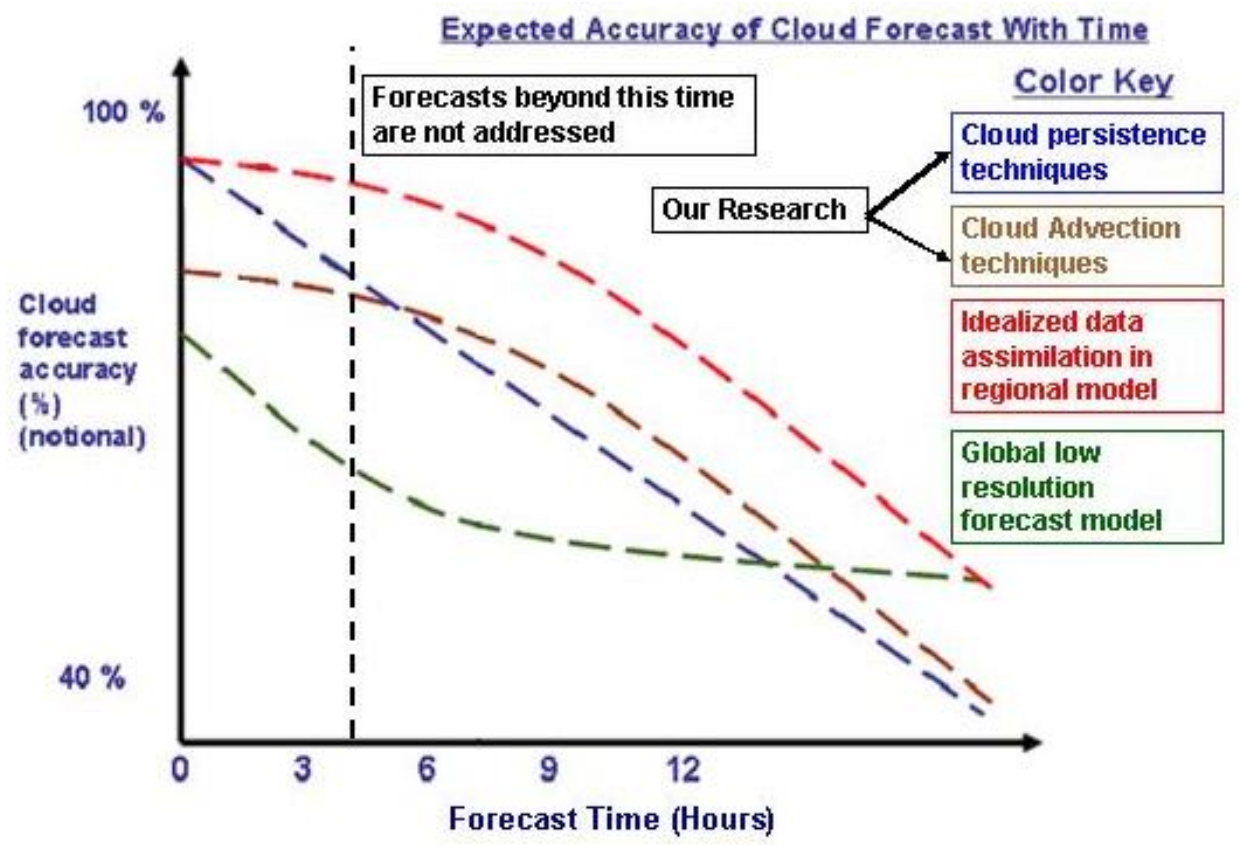

Fig 2: Graph of idealized cloud forecast skill for various forecast methods with time. 


\section{LITERATURE REVIEW}

\subsection{Persistence Cloud Nowcasting Methods}

Although cloud nowcasting has posed a significant problem for both civilian and military forecasters, very little research has been conducted in this area in the past decade. Perhaps what can be described as one of the earliest methods for cloud nowcasting are cloud climatologies. In 1982, the World Climate Research Programme established the International Satellite Cloud Climatology Project in an attempt to understand how the radiative properties of clouds affect the world's climate (ISCCP 2008). The project began gathering satellite data from the five geostationary satellites in orbit in 1983 and began creating mosaic images every three hours at $30 \mathrm{~km}$ resolution. Average cloud cover percentages on a monthly and yearly basis can be represented on maps for scientific study (Figure 3).

Reinke et al. (1990) recognized the potential forecasting applications of cloud climatologies. Using GOES-East 1-2 km resolution data over the state of Florida, they produced a composite of one month of cloud data between the hours of $1500 \mathrm{Z}$ and 2100Z. Treating the $1500 \mathrm{Z}$ period as the initial condition and the $2100 \mathrm{Z}$ as the forecast period, cloud conditional frequency composites were produced that dictated where the highest probabilities of cloud at $2100 \mathrm{Z}$ are given cloudy conditions at $1500 \mathrm{Z}$ and where the highest probabilities of cloud at $2100 \mathrm{Z}$ are given clear conditions at $1500 \mathrm{Z}$. This climatologically-based forecast method is also performed for our domain to serve as a comparison, as described in section 4.6. Moreover, because of the short time frame 


\section{ISCCP-D2 Monthly Mean for June 1991}

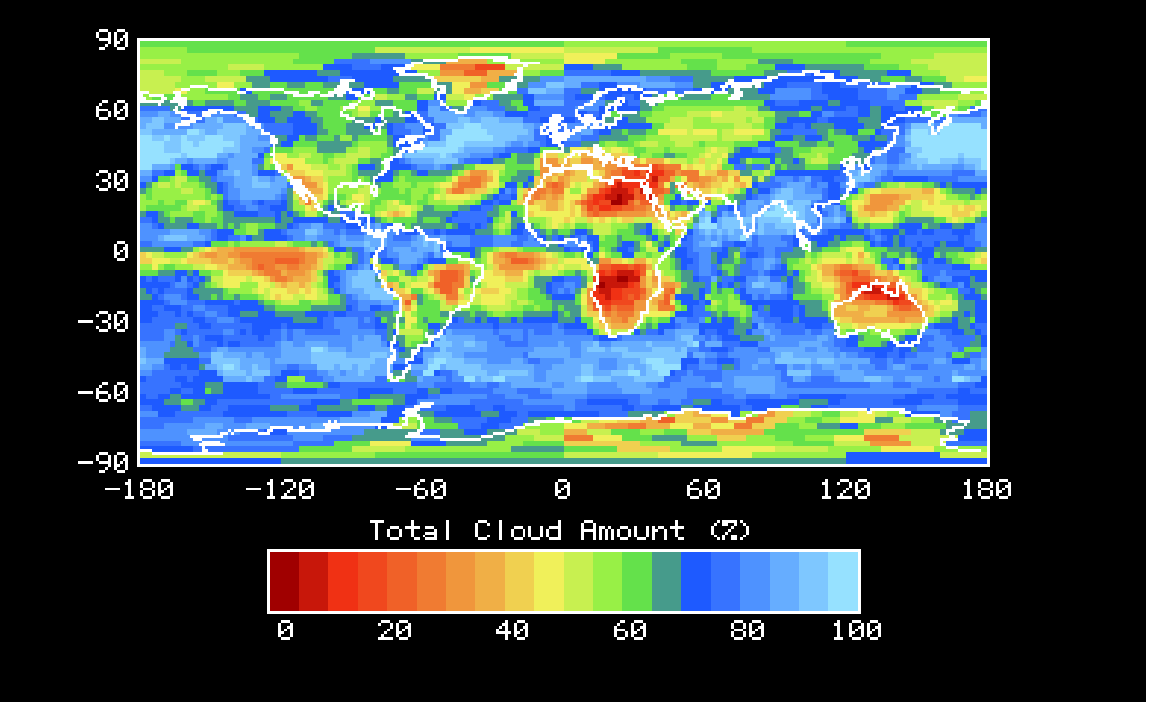

Fig 3: Example of mean cloud percentage map created by the ISCCP for June 1991 (from ISCCP 2008).

during which Reinke et al. forecasted clouds (six hours), it is important to note that this forecast was essentially a nowcasting technique. That is, the frequency of cloud occurrence at $2100 \mathrm{Z}$ could be looked at as a reasonable first guess for a cloud/no-cloud forecast for any given location. It was concluded that cloud frequency climatologies can serve as a useful forecast tool.

Hall et al. (1997) expanded this concept by utilizing the Climatological and Historical Analysis of Clouds for Environmental Simulations (CHANCES) database over Yugoslavia. CHANCES provided 5-km hourly satellite data in the form of binary cloud/no-cloud information through complex preprocessing and compositing. Unlike Reinke et al. focusing on one six-hour period over Florida for one month, a year of data 
was processed over Yugoslavia and conditional probabilities for all twenty-four hours of a day were calculated for Bosnia and Herzegovina's capital city of Sarajevo.

If clouds exist over the city at any time of day, the probability of the sky still being cloudy was calculated for any successive hour, up to twelve hours. This is a significant result in terms of cloud nowcasting because a "cloud forecast matrix" was created, giving a forecaster an additional tool with which to issue cloud-cover forecasts. In addition, many remote areas are in need of cloud forecasts, yet have no forecasters of their own on site. The ability to issue forecasts using only remote sensing instrumentation only is both convenient and practical. Furthermore, Hall et al. noticed that the quality of a persistence forecast decreases with time during the summer months if the initial condition is clear due to the convective diurnal cycle of cloud cover in the region. This ability to detect atmospheric signals from conditional cloud climatologies further confirmed their value. 


\subsection{Displacement Cloud Nowcast Methods}

Although persistence cloud nowcasts are valuable and often have good forecast skill, displacement cloud nowcast methods are another methodology pursued by many researchers. These methods involve treating clouds as passive tracers of the ambient wind, which moves them in the appropriate speed and direction. In order to accomplish this, the ambient wind field must be observed, often in regions where atmospheric soundings are unavailable (i.e. over oceans). The idea of inferring atmospheric motion from cloud movement dates to the very first satellite animation of clouds, produced with the launch of the TIROS polar orbiting satellite in 1960 (Menzel, 2000). In 1963, Fujita and Uchijima studied the cloud movement of a South Pacific tropical storm to infer lowlevel wind direction and vertical wind shear. However, accurate wind speed information eluded their study.

When the first geostationary satellite, Applications Technology Satellite One (ATS-1), was launched in 1966, cloud motion vectors were finally within reach. While Bradbury and Fujita (1968) attempted to infer such vectors using ground-based cameras only, the spatial limitations inherent in this approach proved to be impractical. One of the first cloud motion vector studies was conducted by Fujita (1968) who analyzed a subtropical jet stream, using this information to compute dynamical properties such as vorticity and divergence associated with the relative wind maximum. Various studies using cloud motion vectors were conducted during the late 1960s and early 1970s, using very time-consuming methods involving manually tracking the cloud movement on paper and inferring wind speed (Kidder and Vonder Haar 1995). However, this methodology was greatly improved upon with the onset of the computer age. 
Leese et al. (1971) sought to turn cloud motion vector identification into an automated process through a cross-correlation method. This technique uses computers to identify cloud features and, using the assumption that the brightness temperature of the clouds does not change, finds that same feature in a successive image, allowing for wind speed and direction information to be calculated. Results were compared to various ground-truth wind estimates and shown to capture the overall wind speed quite well. However, wind direction calculations were deemed to be less accurate for the automated technique than for the manual technique. Furthermore, the automated technique required a great deal of computing power and performed poorly in areas of multiple cloud layers. Since then, automated cloud motion vectors improved substantially. Currently, they are produced every three-hours by the National Oceanic and Atmospheric Administration (NOAA)'s National Environmental Satellite, Data, and Information Service (NESDIS) using both infrared and water vapor imagery (Figure 4). Visible-wavelength cloud motion vectors are also produced during daytime hours over the GOES satellite's domain (Dills and Smith 1992). These cloud motion vectors are improving forecasting by being assimilated into current generation forecast models, but Hamill and Nehrkorn (1993) suggested a different way in which to improve forecasting. Building upon the crosscorrelation method, they speculated that cloud motion vectors could also be used to forecast cloud cover and location in the near future. Specifically designed for nowcasting clouds, cloud motion vectors were derived for a specific region and assumed to be constant in time when producing a forecast image of cloud cover. Verification data was taken from the GOES-East satellite at $11 \mathrm{~km}$ resolution approximately zero to 2.5 hours later (in half-hour increments) to determine how the forecast performed. It was shown 


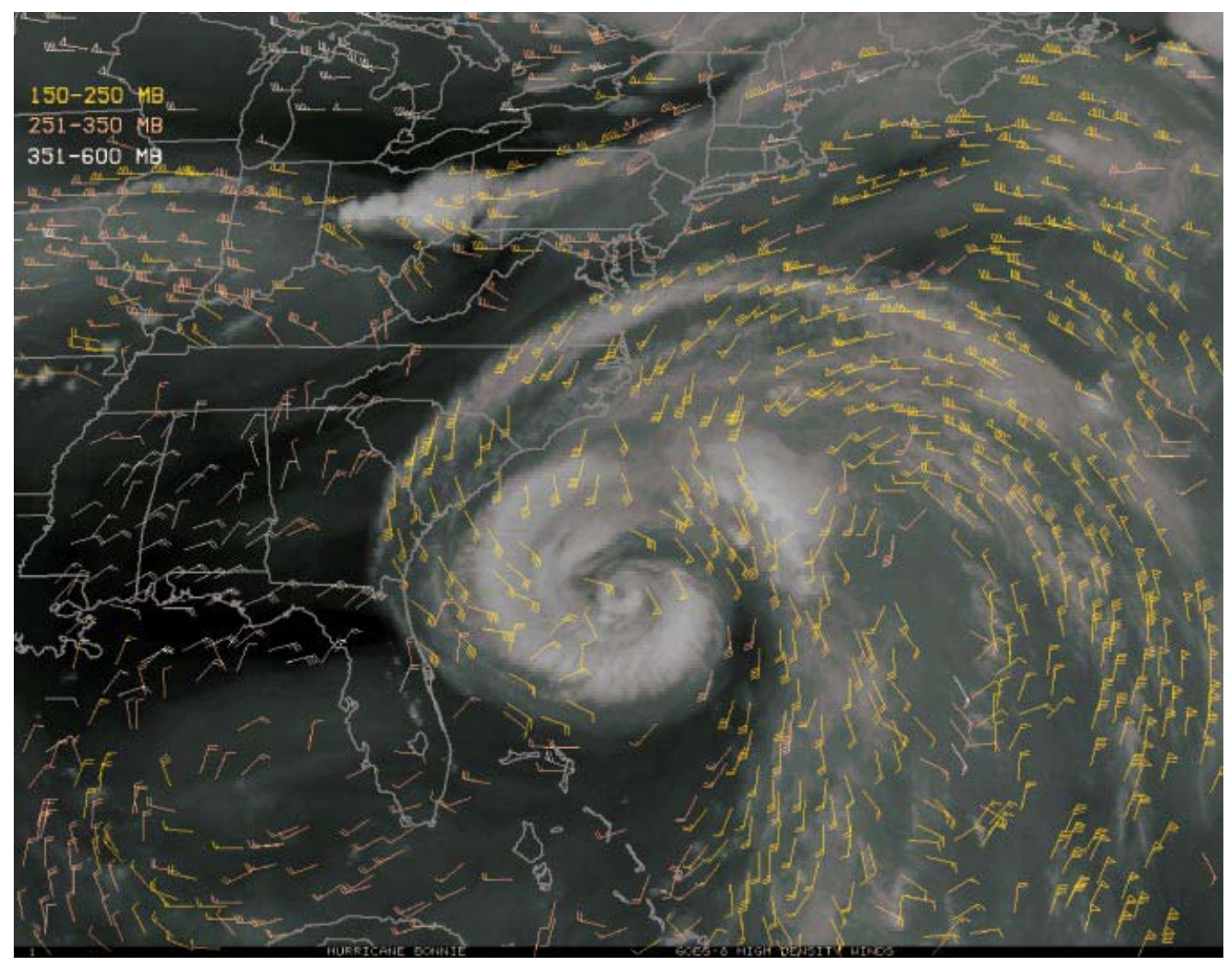

Fig 4: Example of cloud motion vectors produced by the GOES-East satellite on for Hurricane Bonnie on 25 August 1998. Heights of wind vectors are indicated by color (reproduced from Menzel 2000).

that a cross-correlation forecast method improves upon a persistence forecast. It was also shown to perform better than displacing all the clouds along at both the $500 \mathrm{mb}$ wind speed and the $700 \mathrm{mb}$ wind speed. This was the first study to use cloud motion vectors as a direct predictor of cloud cover, instead of simply providing input for a forecast model.

One of the most substantial efforts to produce cloud cover nowcasts was undertaken by the United States Air Force in the 1990s. Their cloud forecast model, Advect Cloud (ADVCLD), was created because of the armed forces' need for quality 
short-term cloud forecasts for mission planning purposes. ADVCLD uses initial wind and moisture fields from the Navy Operational Global Atmospheric Prediction System (NOGAPS) and interpolates them to a 25 nautical-mile grid in three-hour forecast intervals (Kopp et al. 1997). Unlike cloud motion vectors, which assume the pressure of the cloud remains constant, ADVCLD calculates a displacement in the vertical direction as well. This incorporation of NOGAPS data into an advection model has yielded good results; however the term "good" is subjective. The results presented by Kopp et al. emphasize low Root Mean Square Errors on a hemispheric plot of the entire planet. The resolution of this display is incredibly coarse, able to capture only the synoptic scale cloud features. One would expect advection of clouds to perform well in coarseresolution because individual cloud entities are washed out by the large-scale system's progression. In terms of mesoscale cloud movement, ADVCLD needs much improvement.

Very little research has been conducted in the last ten years focusing exclusively on cloud motion vectors and how to improve them. Instead, cloud motion vectors are being used to assist in forecasting other weather conditions, most commonly precipitation. Grose et al. (2001) conducted a study over northern South America and the Caribbean to attempt to nowcast precipitation amounts using GOES IR data. To do this, not only did a rain-rate probability matching method need to be developed to associate certain equivalent brightness temperatures with rainfall, but a cloud motion vector scheme was also used. Very similar to the cross-correlation method previously described, the authors found that rather than leaving gaps in the wind field in their domain (since cloud motion vectors can only be determined in the presence of clouds), 
interpolating and smoothing the wind to cover the entire domain further improved their forecasting skill. A Monte Carlo averaging system was also employed to filter "bad" cloud motion vectors.

Joyce et al. (2004) performed a similar precipitation/cloud motion study to that of Grose et al., except that they applied it on a planetary scale using all five geosynchronous satellites in orbit. The authors yielded good results; however, the coarse resolution again may have smoothed out individual cloud features.

The tracking of mesoscale convective systems and monsoon rains is also a topic of study using cloud motion vectors. These systems are large enough and have cloudtops cold enough that they can be tracked even if they undergo fundamental changes in structure (change shape, split into multiple systems, etc.) (Williams and Houze 1987). 


\subsection{Additional Cloud Nowcasting Methods}

Cloud nowcasting techniques not related to persistence or displacement methods are beyond the scope of the study performed in this thesis. Nevertheless, it is important to acknowledge that there are additional methodologies to tackle the cloud nowcasting problem, some of which yield impressive results.

A strictly statistics-based methodology to predict cloud cover was used by Norquist (2000). Using over one hundred potential variables from numerical weather prediction model forecasts, a search was performed to find statistical relationships between the variable in question and any cloud variable. This approach was used because of the Air Force's need for operational cloud cover predictions on the ground, something difficult to do using satellite data. Results from their Diagnostic Cloud Forecast showed better results than either persistence or ADVCLD forecasts over the same time period over the northern hemisphere, both in cloud cover and cloud layer altitude. However, because the initialization data from the NOGAPS model must be interpolated to smaller grids for this method to work, predictor satellite images created using this method look a bit coarse.

An alternative to a strictly statistics-based methodology is an analog method used by Hall et al. (2010) using a combination of both statistics and past weather patterns. Using a five-year database over various domains in the U.S., pattern recognition was used to determine the cloud cover over certain cities out to five hours based on past observed outcomes. Although this algorithm needs to be trained depending on the target location for cloud cover, the time it takes to do this is negligible, and improvement upon persistence and climatological-expectancy-of-persistence is significant. 
Solar radiation forecasts can also benefit from cloud nowcasting, as exhibited by Modica et al. (2010). The method utilized is similar in that cross-correlations are used, however cloud properties are also taken into account, including cloud top, cloud base, and cloud optical depth. Knowledge of cloud level allows for cloud motion vector fields to be calculated for both high clouds and low clouds simultaneously (rather than applying one gridded cloud motion vector field to clouds at all heights). Coupled with a radiative transfer model, this methodology allows for solar downward flux calculations to be calculated one hour in advance over a specific target area, an invaluable resource to solar energy companies as well as weather forecasters predicting the surface temperature. 


\section{MODIS SENSOR}

\subsection{MODIS Cloud Mask Algorithm}

The following sections will describe various algorithms written for the Moderate Resolution Imaging Spectroradiometer (MODIS) sensor that are vital to this research. They include the MODIS Cloud Mask Algorithm, the MODIS Cloud-Top Properties Algorithm, and the MODIS Cloud Phase Algorithm. The Terra and Aqua satellites are equipped with a number of Earth Observing System (EOS) sensors, including MODIS. The Terra satellite was launched by the National Aeronautics and Space Administration (NASA) in December 1999. A polar-orbiting satellite, Terra has a descending orbit with an equatorial crossing of 1030 local solar time (Menzel et al. 2007). In May 2002, NASA launched its Aqua satellite in an ascending orbit with an equatorial crossing of 1330 local solar time. Aqua was the first satellite in a planned constellation of EOS satellites, called the Afternoon Constellation (A-Train).

The MODIS sensor takes measurements at 36 different wavelengths (between 0.42 and $14.24 \mu \mathrm{m}$ ) to derive information about both clouds and the Earth's surface. The vertical resolution of each of these 36 bands varies vertically from 250 meters to 1 kilometer. Several useful algorithms have been derived from these spectral bands, including information about cloud phase as well as the presence of clouds. It is common knowledge that passive satellite sensors do not directly detect clouds. Rather, radiances are measured at different wavelengths and interpreted as a brightness temperature (Kidder and Vonder Haar 1995). In many cases, clouds are distinguished in these infrared images by their colder brightness temperatures than the ground, with clouds 
higher in the atmosphere having the colder brightness temperatures. This is not always the case. For example, if the surface is covered with ice or snow, distinguishing between low cloud and the ground from brightness temperature alone can be difficult, if not impossible, from brightness temperatures alone. In addition, there is often little contrast between surface radiances and low stratus or cumulus clouds during the nighttime hours (Ackerman et al. 2010).

Since the MODIS sensor measures many more wavelengths than the GOES satellites (36 channels as opposed to 5 channels on GOES), much more radiance information is available. Taking advantage of 19 of its spectral bands, a cloud detection algorithm (called the Cloud Mask/MOD35) has been developed (Ackerman et al. 2010), as shown in Table 1. Created by the MODIS Cloud Mask Team at the Cooperative Institute for Meteorological Satellite Studies (CIMSS) at the University of Wisconsin Madison, the MODIS Cloud Mask is considered to be the one of best cloud mask algorithms currently in operation.

The MODIS Cloud Mask is available in both one-kilometer and five-kilometer resolutions. Each field-of-view (FOV) is assigned a confidence value between 0 and 1 , where 1 represents high confidence in clear conditions, 0 represents high confidence in cloudy conditions, and the numbers in between represent increasingly less confidence in cloudy or clear conditions as 0.5 is approached (Ackerman et al. 2010). As a result, the final Cloud Mask output is given four levels of unobstructed FOV confidence: cloudy, uncertain clear, probably clear, and confident clear.

The Algorithm Theoretical Basis Document (ATBD) for the MODIS Cloud Mask (Ackerman et al. 2010) clearly explains the thresholds and processes for each spectral 
Table 1: MODIS spectral bands. The Y/N column denotes whether or not each band is used in the Cloud Mask algorithm (reproduced from Ackerman et al. 2010).

\begin{tabular}{|c|c|c|c|}
\hline \multirow{2}{*}{$\begin{array}{c}\text { Band } \\
1(250 \mathrm{~m})\end{array}$} & $\begin{array}{c}\text { Wavelength } \\
(\mu \mathrm{m})\end{array}$ & \multicolumn{2}{|r|}{ Comment } \\
\hline & 0.659 & $\mathrm{Y}$ & (250 $\mathrm{m}$ and $1 \mathrm{~km}$ ) clouds, shadow \\
\hline $2(250 \mathrm{~m})$ & 0.865 & $\mathrm{Y}$ & $(250 \mathrm{~m}$ and $1 \mathrm{~km})$ low clouds \\
\hline $3(500 \mathrm{~m})$ & 0.470 & $\mathrm{~N}$ & \\
\hline $4(500 \mathrm{~m})$ & 0.555 & $\mathrm{Y}$ & \\
\hline $5(500 \mathrm{~m})$ & 1.240 & $\mathrm{Y}$ & Snow \\
\hline $6(500 \mathrm{~m})$ & 1.640 & $\mathrm{Y}$ & snow, shadow \\
\hline $7(500 \mathrm{~m})$ & 2.130 & $\mathrm{Y}$ & \\
\hline 8 & 0.415 & $\mathrm{~N}$ & \\
\hline 9 & 0.443 & $\mathrm{~N}$ & \\
\hline 10 & 0.490 & $\mathrm{~N}$ & \\
\hline 11 & 0.531 & $\mathrm{~N}$ & \\
\hline 12 & 0.565 & $\mathrm{~N}$ & \\
\hline 13 & 0.653 & $\mathrm{~N}$ & \\
\hline 14 & 0.681 & $\mathrm{~N}$ & \\
\hline 15 & 0.750 & $\mathrm{~N}$ & \\
\hline 16 & 0.865 & $\mathrm{~N}$ & \\
\hline 17 & 0.905 & $\mathrm{Y}$ & \\
\hline 18 & 0.936 & $\mathrm{Y}$ & low cloud detection \\
\hline 19 & 0.940 & $\mathrm{Y}$ & \\
\hline 26 & 1.375 & $\mathrm{Y}$ & thin cirrus, high cloud \\
\hline 20 & 3.750 & $\mathrm{Y}$ & shadow \\
\hline $21 / 22$ & 3.959 & $\mathrm{~N}(21) / \mathrm{Y}(22)$ & window, shadow \\
\hline 23 & 4.050 & $\mathrm{Y}$ & \\
\hline 24 & 4.465 & $\mathrm{~N}$ & \\
\hline 25 & 4.515 & $\mathrm{~N}$ & \\
\hline 27 & 6.715 & $\mathrm{Y}$ & high cloud, polar inversions \\
\hline 28 & 7.325 & $\mathrm{Y}$ & cloud at night over land with $\mathrm{B} 31$ \\
\hline 29 & 8.550 & $\mathrm{Y}$ & brightness temperature difference \\
\hline 30 & 9.730 & $\mathrm{~N}$ & \\
\hline 31 & 11.030 & $\mathrm{Y}$ & window threshold \\
\hline 32 & 12.020 & $\mathrm{Y}$ & brightness temperature difference \\
\hline 33 & 13.335 & $\mathrm{Y}$ & polar regions, used with B31 \\
\hline 34 & 13.635 & $\mathrm{~N}$ & \\
\hline 35 & 13.935 & $\mathrm{Y}$ & high cloud test \\
\hline 36 & 14.235 & $\mathrm{~N}$ & \\
\hline
\end{tabular}

test. For convenience, one such spectral test will be described here as an example. Bit 15 in the Cloud Mask Algorithm involves the $6.7 \mu \mathrm{m}$ water vapor channel. Used to detect the presence of high clouds, it has been well-established that clear-sky radiation emitted at $6.7 \mu \mathrm{m}$ between 200 and 500 millibars is indicative of the temperature and moisture in the layer below it (Soden and Bretherton 1993). When present, high clouds at this vertical level have colder brightness temperatures than the surrounding pixels. Therefore, 
thresholds of $215 \mathrm{~K}, 220 \mathrm{~K}$, and $225 \mathrm{~K}$ have been established for high confidence, midpoint, and low confidence of cloud cover, respectively.

Furthermore, this test uses a difference of the brightness temperature at the 11.0 $\mu \mathrm{m}$ infrared channel and the $6.7 \mu \mathrm{m}$ water vapor channel to diagnose clear-sky over the polar regions at night. That is, $\mathrm{BT}_{11.0}-\mathrm{BT}_{6.7}$ has been shown to be largely negative in polar regions during nighttime hours due to the strong surface inversion that develops. This difference is nearly always positive in other parts of the world since the upper troposphere (where most of the $6.7 \mu \mathrm{m}$ radiation is coming from) is nearly always colder than near the surface (where most of the $11.0 \mu \mathrm{m}$ radiation is coming from). In order to avoid incorrectly placed high, cold clouds in cloud-free areas of the Arctic, a threshold value of less than $-10^{\circ} \mathrm{C}$ has been established for this spectral test. 


\subsection{MODIS Cloud-Top Properties Algorithm}

A second product used extensively in this research is the MODIS Cloud-Top Properties Algorithm/MOD06 (Menzel et al. 2006). Utilizing the $\mathrm{CO}_{2}$ slicing technique (Smith and Platt, 1978), cloud-top properties (including cloud-top pressure and temperature) are calculated for each cloudy pixel (as identified by the Cloud Mask algorithm). The $\mathrm{CO}_{2}$ slicing technique results from the opacity of the atmosphere to outgoing radiation from 13.3 to $15.0 \mu \mathrm{m}$ due to absorption by carbon dioxide. There are four spectral bands in this so-called "dirty window" (due to its contamination by $\mathrm{CO}_{2}$ ) available on MODIS (Figure 5). Channel 33 senses radiation at $13.34 \mu \mathrm{m}$, Channel 34 at $13.64 \mu \mathrm{m}$, Channel 35 at $13.94 \mu \mathrm{m}$, and Channel 36 at 14.24

$\mu \mathrm{m}$. Each of these channels has a weighting function (Kidder and Vonder Haar 1995) that peaks at $900,700,500$, and $300 \mathrm{mb}$ respectively.

The ability to derive cloud-top pressure for a given cloud pixel is given by Equation (1), which describes a radiance ratio between two MODIS channels.

$$
\frac{R\left(v_{1}\right)-R_{c l r}\left(v_{1}\right)}{R\left(v_{2}\right)-R_{c l r}\left(v_{2}\right)}=\frac{N \cdot E_{1} \int_{P_{S}}^{P_{C}} \tau\left(v_{1}, p\right) \frac{d B\left[v_{1}, T(p)\right]}{d p} d p}{N \cdot E_{2} \int_{P_{S}}^{P_{C}} \tau\left(v_{2}, p\right) \frac{d B\left[v_{2}, T(p)\right]}{d p} d p}
$$

$R\left(v_{1}\right)$ and $R\left(v_{2}\right)$ are the observed radiances at two successive bands in the $\mathrm{CO}_{2}$ MODIS window (frequencies $v_{1}$ and $\left.v_{2}\right), R_{c l r}\left(v_{1}\right)$ and $R_{c l r}\left(v_{2}\right)$ are the clear-sky radiances at two consecutive $\mathrm{CO}_{2}$ window frequencies, $N$ is the fraction of the field of view covered by cloud, $E_{1}$ and $E_{2}$ are the emissivities at frequencies $v_{1}$ and $v_{2}, P_{C}$ is the cloud 


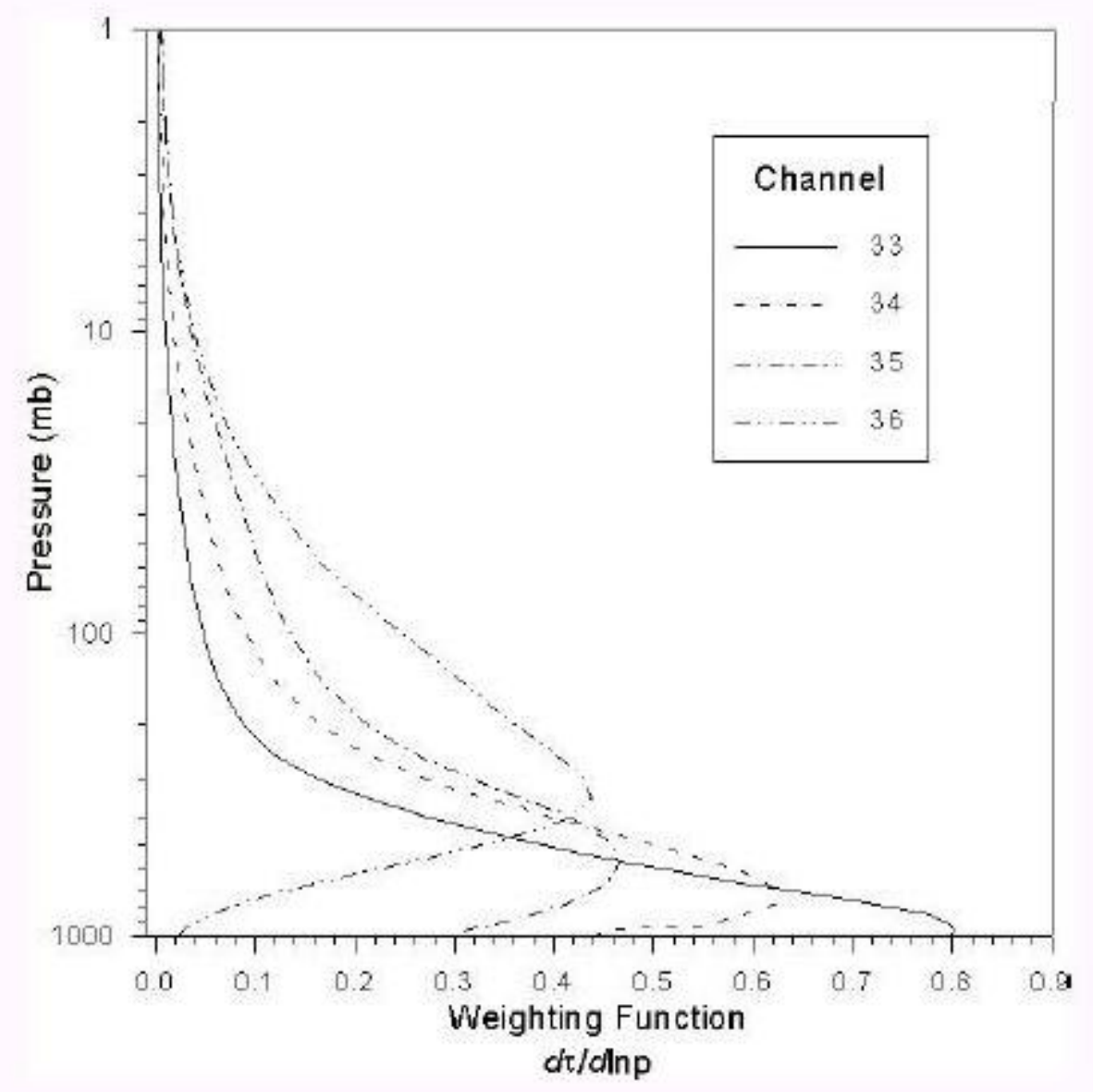

Fig 5: The four $\mathrm{CO}_{2}$ channels on the MODIS sensor and their weighting functions (reproduced from Menzel et al. 2002).

pressure, $P_{S}$ is the surface pressure, $\tau(v, p)$ is the fractional transmittance of radiation at frequency $v$ arriving at the top of the atmosphere $(p=0)$ from pressure level $p$, and $B[v, T(p)]$ is the Planck radiance at temperature $T(p)$ for frequency $v$ (Menzel et al, 2006).

Cloud-top pressures are calculated using the assumption that for frequencies closely spaced in wavenumber (e.g. MODIS Channel 36 and MODIS Channel 35), the emissivities at the two frequencies are essentially equal. To yield the clear-sky radiances 
on the left side of Equation (1), a transmittance model using radiative transfer calculations for the four MODIS $\mathrm{CO}_{2}$ bands is incorporated to account for gas concentration and satellite viewing angle (Menzel et al. 2007). To solve the right side of the equation, temperature and moisture profiles from the Global Forecast System model (GFS), in addition to vertical profiles of atmospheric transmittance in the four $\mathrm{CO}_{2}$ bands (from the previously mentioned transmittance model) as a function of cloud-top pressure.

Once these variables are available, a "top-down" approach is utilized to find cloud-top pressure. That is, the two MODIS $\mathrm{CO}_{2}$ spectral bands most opaque to radiation (Channels 36 and 35) are used in Equation (1) first. If the solution yields a pressure high in the atmosphere, that is what the cloud-top pressure is said to be and no other spectral ratios are performed. If no solution is found, the next such ratio between Channels 35 and 34 is used, followed by 34 and 33 if no solution is found. It should be noted that only the MODIS sensor on the Aqua satellite performs these three tests to calculate cloud-top pressure. Because Channel 34 has noise problems on the Terra satellite, only ratios of Channels $36 / 35$ and $35 / 33$ are used. In addition, this product is averaged over a $5 \times 5$ pixel box, thus yielding a resolution of $5 \mathrm{~km}$. Additional information is provided in the Cloud Top Properties and Cloud Phase ATBD (Menzel et al. 2006). 


\subsection{MODIS Cloud Phase Algorithm}

The final MODIS algorithm utilized in this research is the MODIS Cloud Phase Algorithm/MOD06 (Menzel et al 2006). The idea for this algorithm began before the first launch of MODIS in 1999. Strabala et al. (1993) proposed a trispectral (that is, three spectral absorption bands) technique to use measurements from the future MODIS sensor to delineate cloud phase. While the theory behind their proposal forms the basis of the actual MODIS Cloud Phase Algorithm, it will be shown that changes had to be made with the actual data calculations.

It is apparent that clouds in different phases (i.e. liquid water, ice, etc.) have different radiative properties from one another. In addition to particle size, the absorption and emission of radiation by clouds depend upon the cloud particle's index of refraction. Equation (2) describes the index of refraction $m$.

$$
m=n_{r}-i n_{i}
$$

$n_{r}$ represents the real part of the equation, indicative of the particle's scattering strength. $i n_{i}$ is the imaginary part, indicative of the particle's absorptive properties (Menzel et al. 2006). Figure (6) shows both the real and imaginary parts of the index of refraction for both ice and liquid water particles between 8 and $13 \mu \mathrm{m}$ wavelengths, assuming similar temperature and similar microphysical size and shape distributions. Note that the particles' absorptive properties are very similar, regardless of phase, between 8.5 and $10.0 \mu \mathrm{m}$. However, between 10.0 and $13.0 \mu \mathrm{m}$, ice particles absorb much more radiation 


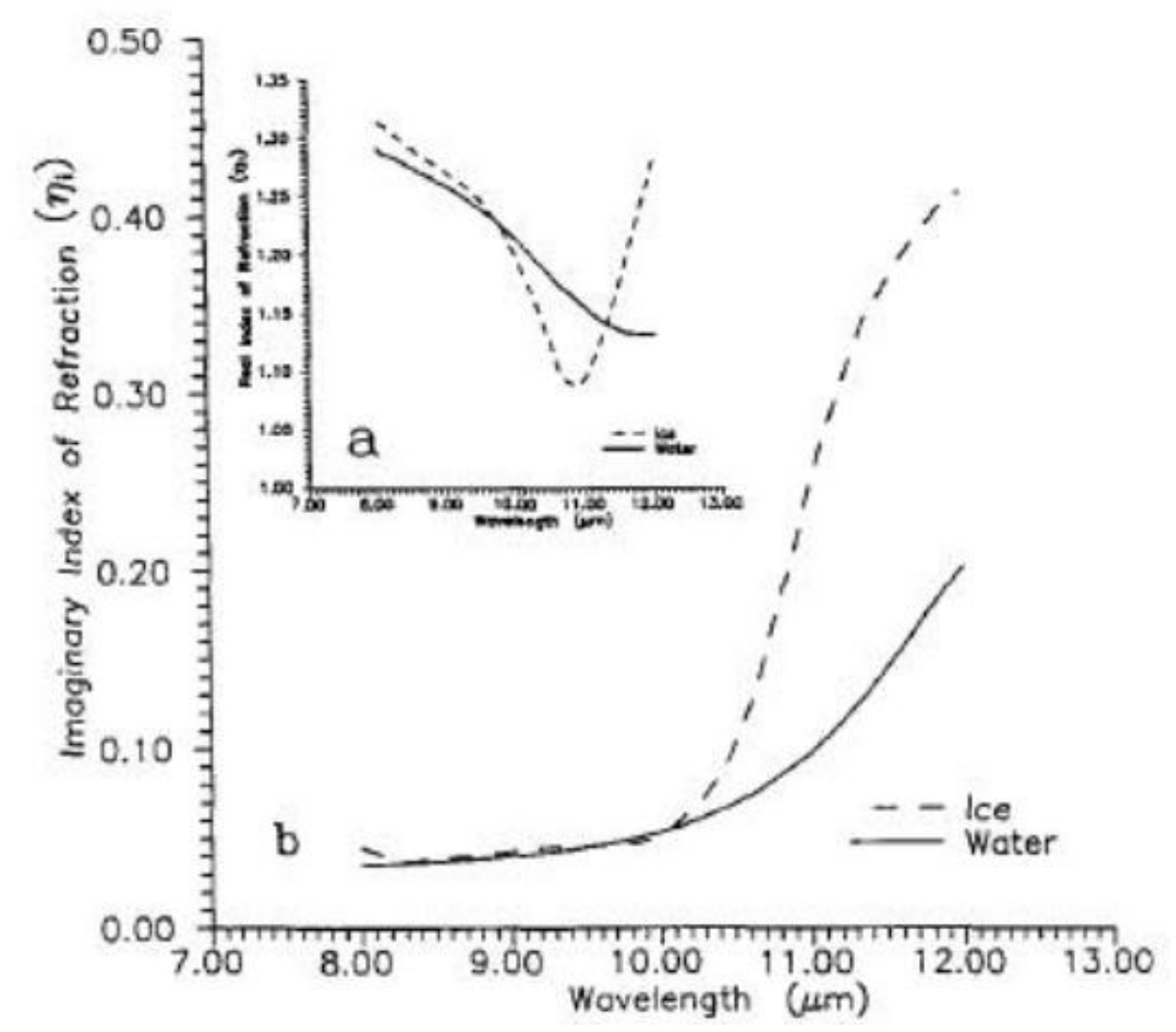

Fig 6: The scattering (real) and the absorptive (imaginary) parts of the index of refraction for both ice and water particles (reproduced from Strabala et al. 1993).

than water particles, thus forming the original basis for Strabala et al.'s trispectral technique.

This finding, although significant, is not used in the MODIS Cloud Phase Algorithm. Ackerman et al. (1990) noticed that large positive brightness temperature between 8.5 minus $11 \mu \mathrm{m}$ were indicative of cirrus clouds, since the absorptive properties of ice are greater than that of water. However, incorporation of the 11 minus $12 \mu \mathrm{m}$ brightness temperature differences proved unnecessary since the differences are similar in magnitude and sign as the 8.5 minus $11 \mu \mathrm{m}$ differences. Thus, the 11 minus $12 \mu \mathrm{m}$ 
difference is not used in the MODIS Cloud Phase Algorithm and the 8.5 minus11 $\mu \mathrm{m}$ brightness temperature difference, as well as the $11 \mu \mathrm{m}$ brightness temperature itself, is used and is considered adequate for cloud phase differentiation.

The MODIS Cloud Phase Algorithm is also averaged over a 5 x 5 pixel box, yielding a resolution of $5 \mathrm{~km}$. Its outputs include: clear sky, water cloud, ice cloud, mixed-phase cloud, and unknown. More information regarding this algorithm is available in the MOD06 ATBD (Menzel et al. 2006). 


\section{METHODOLOGY}

\subsection{Spatial Domain Considerations}

To assess the hypothesis that terrain-induced low clouds often persist over an orographic feature while higher clouds simply displace with the wind, an area containing complex terrain was selected for this study. The State of Utah and portions of southwest Wyoming were chosen for the various mountain ranges as well as the valleys and deserts in order to observe complex cloud behavior. In addition, northwest Utah is home to Dugway Proving Ground, a United States Army facility that houses a large weather station mesonet. Because of this high concentration of weather observations, scientists at the Army Research Lab in White Sands, New Mexico use Dugway to initialize their weather models, thus making it an important area of study for the military. The exact latitude and longitude of our western United States domain is $37^{\circ} \mathrm{N}$ to $42^{\circ} \mathrm{N}$ latitude and $109.5^{\circ} \mathrm{W}$ to $-114.05^{\circ} \mathrm{W}$ longitude (see Figure 7 ). This translates to a domain of approximately $500 \mathrm{~km}$ x $400 \mathrm{~km}$. 


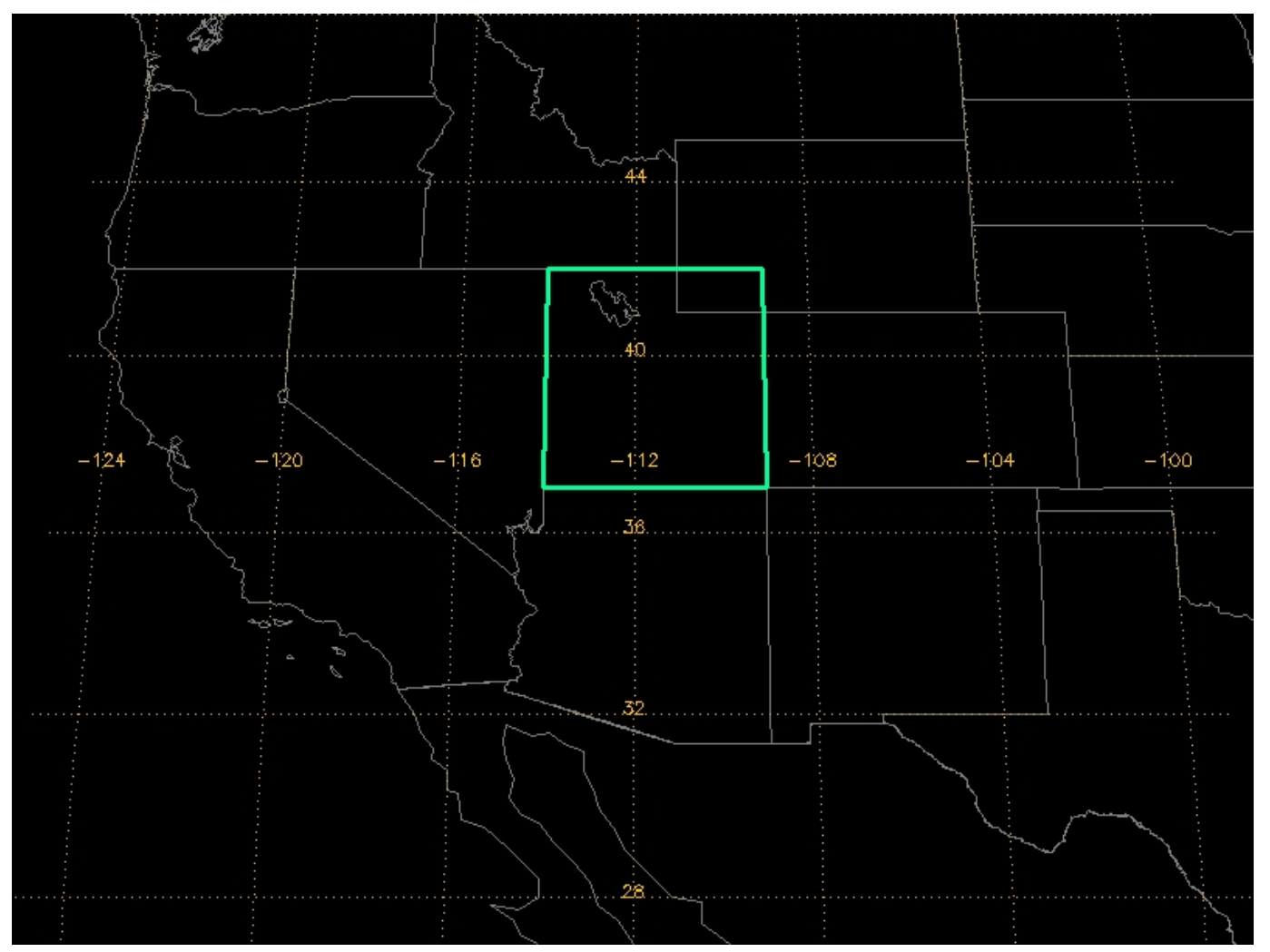

Fig 7: Map of the western United States depicting the evaluation domain over Utah and southwest Wyoming in which all forecasts are evaluated. 


\subsection{Persistence Nowcast Methodology}

This forecast method assumes that clouds do not move between two forecast periods. To make a persistence cloud cover nowcast over our domain, two cloud cover images must be available for comparison. The presence of the MODIS sensor on both the Terra and Aqua satellites provides a unique opportunity to do just that. The Terra satellite passes over the domain at roughly 10:30 local time (depending on the satellite's particular orbit on that day). The domain in question is compared to the Terra scan (called a granule, approximately 2,000 $\mathrm{km}$ wide) intersecting the same area. If the corners of the domain are within 200 meters of the nearest Terra granule point, it is decided that the entire domain is populated by the granule in question. If the granule does not fully populate our domain, that orbit is not used to evaluate a cloud forecast. It is also important to note that, at times, a granule ends before our entire domain is populated. If this occurs, the very next granule is analyzed and it is determined if by piecing the two granules together sequentially, the entire domain can be populated. Because the two granules are separated by five minutes or less, this is satisfactory. However, if the two granules together still do not populate our domain, that orbit is also not used to evaluate a cloud forecast.

Once the domain constraints have been satisfied, the MODIS Cloud Mask at $5 \mathrm{~km}$ resolution is extracted for each grid point. If the Cloud Mask algorithm determines the point to be either "cloudy" or "uncertain clear," a cloud is placed at that point. If the algorithm determines the point to be either "confident clear" or "probably clear", a clear area is placed at that point. If the Cloud Mask was not determined for a particular point, no value is placed at that point. This process is repeated only for the domain in question. 
Next, it must be determined if the MODIS sensor on the Aqua satellite will pass over our domain in a nowcast timeframe. For our research, anything shorter than 4.5 hours falls into this category. The same process described for the Terra MODIS pass is repeated for the Aqua MODIS pass, ensuring that our entire domain is populated. Again, if the domain is not completely populated, the orbit is not used for cloud nowcasting evaluation (even if the Terra MODIS orbit does populate the area).

The two $5 \mathrm{~km}$ Cloud Masks over our domain are then interpolated (Figure 8) to approximately a $1 \mathrm{~km}$ Cloud Mask (Figures 9 and 10). If there is a transition from a cloudy area to a clear area, the distance between the $5 \mathrm{~km}$ resolution cloud area and the $5 \mathrm{~km}$ resolution clear area is halved, with the points closest to the cloudy area being labeled as cloudy and the points closest to the clear area being labeled as clear. Because of the shear number of points in question (over 100,000 per case), this interpolation effect is minimal when computing skill.

A Mollweide map projection is used to display this image with an $800 \times 600$ pixel resolution with boundaries of $35^{\circ} \mathrm{N}$ to $46^{\circ} \mathrm{N}$ latitude and $-107^{\circ} \mathrm{W}$ to $-117^{\circ} \mathrm{W}$ longitude, centered at $-111.5^{\circ}$ longitude (roughly the center of Utah). These settings are what dictate the grid spacing of roughly $1 \mathrm{~km}$ using our interpolation scheme.

Because each grid point in the domain is populated for both MODIS scans, a direct comparison of cloud/clear, cloud/cloud, clear/cloud, and clear/clear points can be performed. This process will be further described in the Forecast Skill Evaluation section; however an example can be seen in Figure (11). 

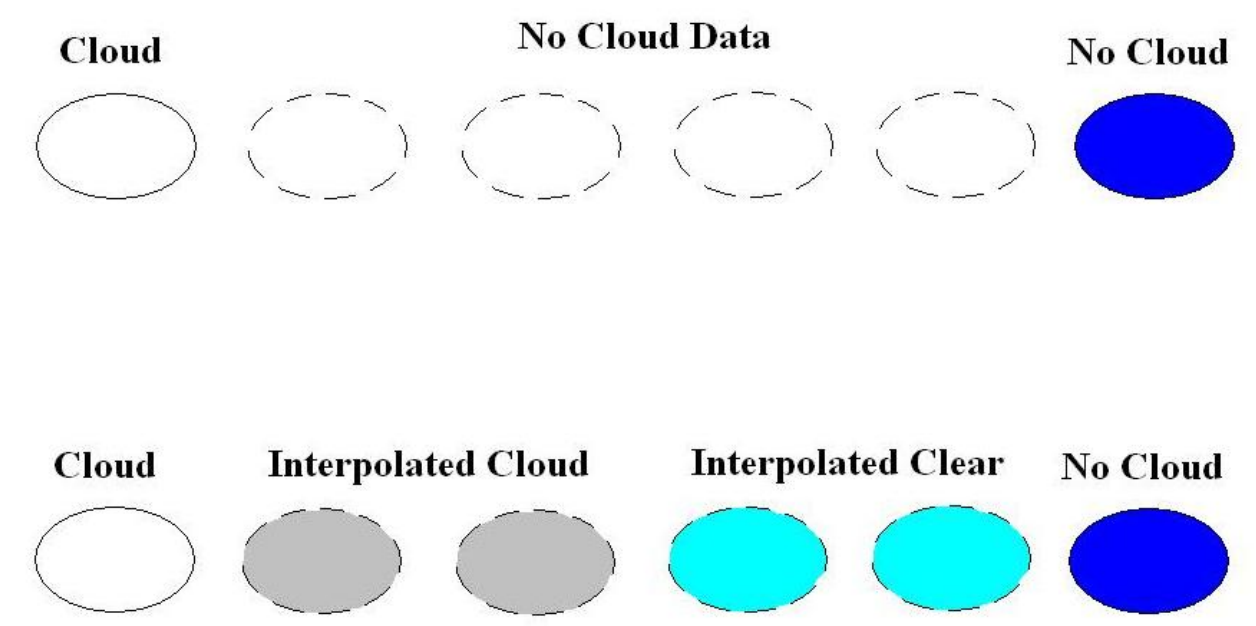

Fig 8: Visual interpretation of cloud mask interpolation scheme from $5 \mathrm{~km}$ (top) to $1 \mathrm{~km}$ (bottom). 

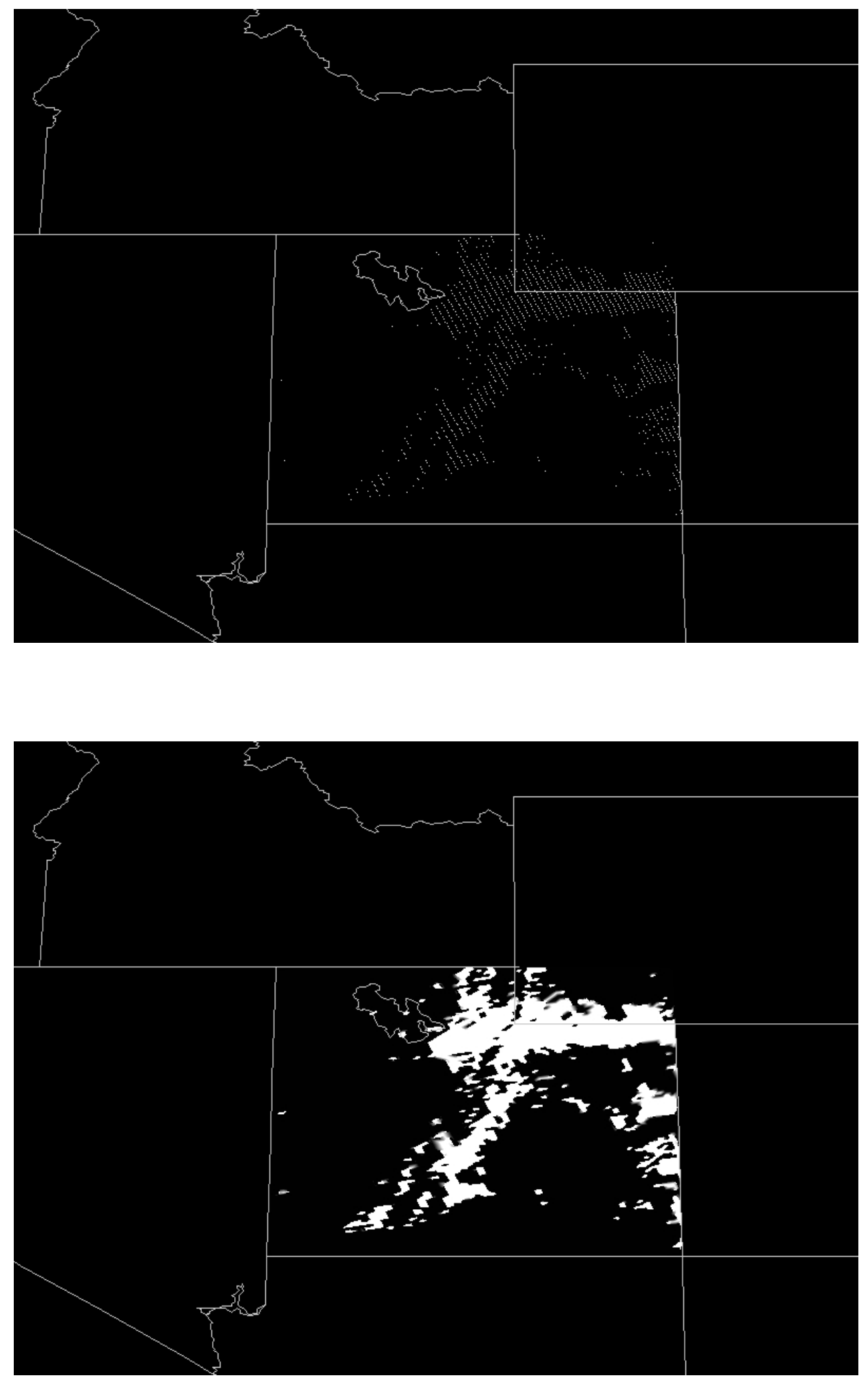

Fig 9: Terra MODIS Cloud Mask swath (top) and interpolated Cloud Mask (bottom) over Utah on 1 December 2008 at 5:05 UTC. 

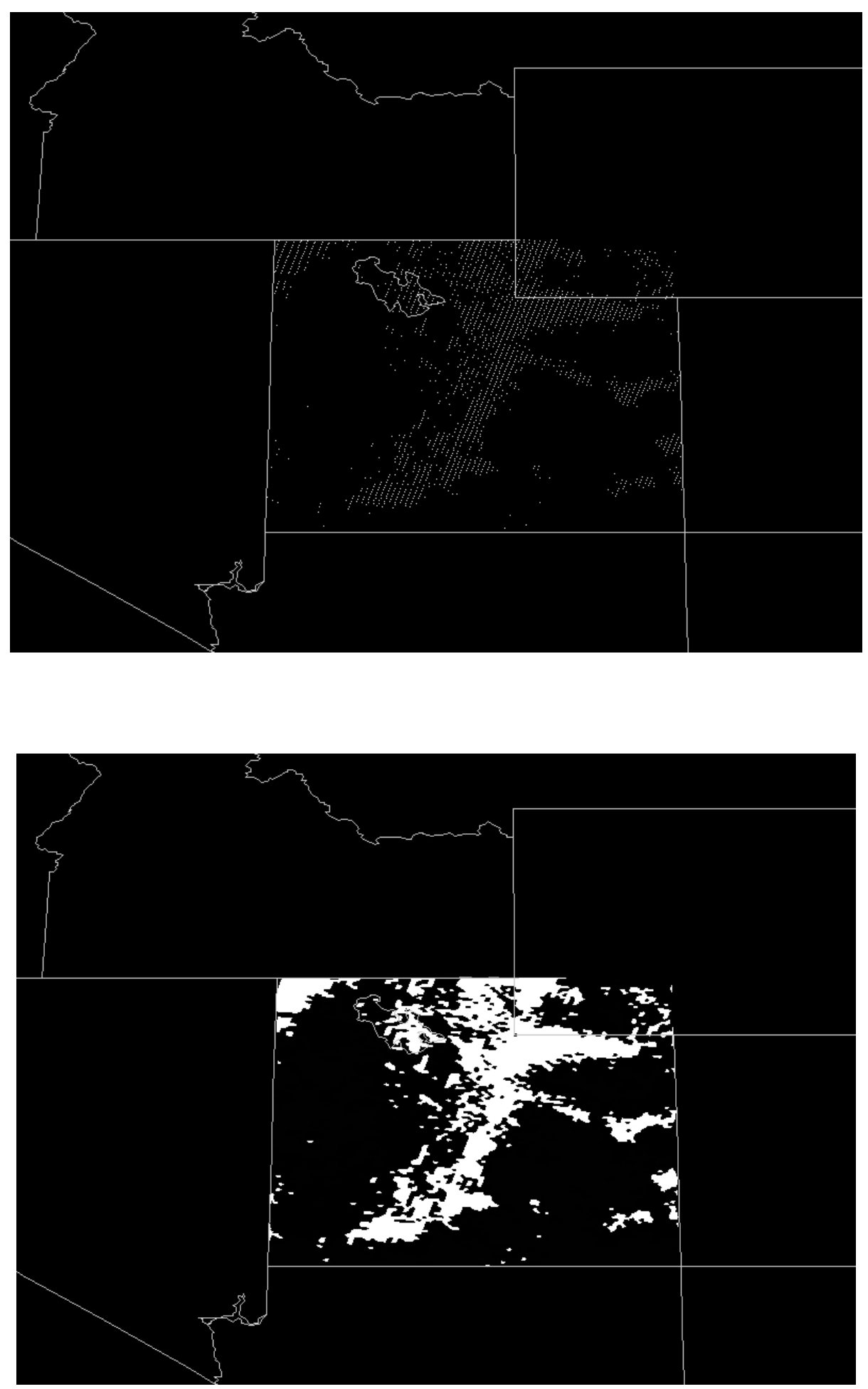

Fig 10: Aqua MODIS Cloud Mask swath (top) and interpolated Cloud Mask (bottom) over Utah on 1 December 2008 at 9:20 UTC. 


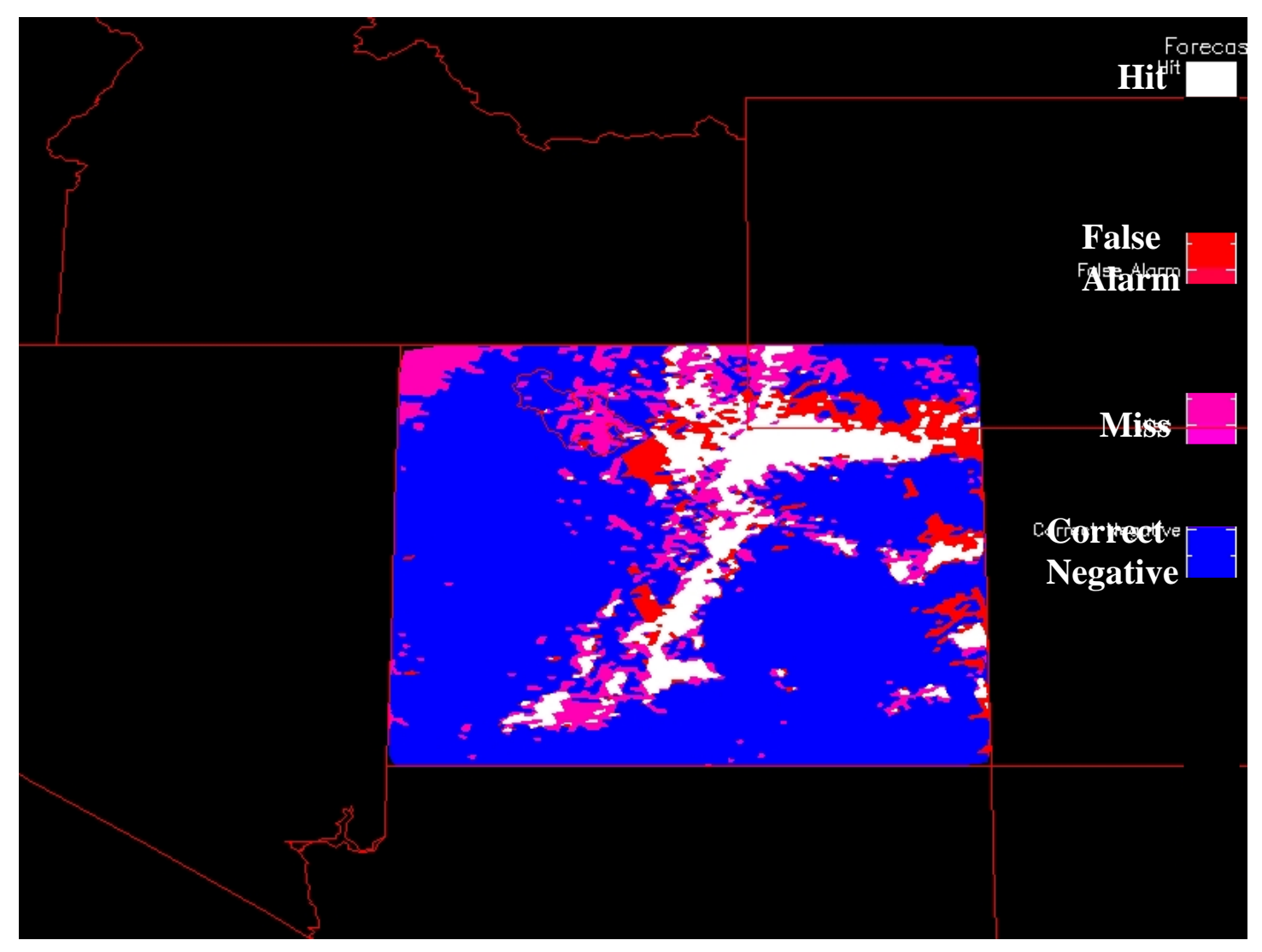

Fig 11: Persistence Method nowcast valid at 9:20 UTC using Figure (9) data as the forecast Cloud Mask and Figure (10) as the validation Cloud Mask. 


\subsection{0mb Wind Displacement Method}

This forecast method assumes that all clouds move at the same speed and in the same direction. The same process as the Persistence Method is repeated so that only cases in which the entire domain is populated are considered. Although the evaluation domain remains the same (that is, the domain over which we assess the cloud nowcast), the domain required to be populated is expanded by two degrees of latitude and two degrees of longitude in all four directions (see Figure 12). This allows for clouds outside of our domain to be displaced into the domain by the mean wind speed. If this were not taken into account, most clouds would simply be displaced out of our domain. With no clouds to replace them, a mostly clear scene would be inaccurately evaluated.

In order to move the clouds accurately, information regarding wind speed and direction is necessary. Originally, GOES satellite-derived winds were going to be used to provide this information. The temporal resolution (every three hours) was ideal for matching with the closest Terra orbit over the area. However, it was soon realized that using such a dataset was more problematic than expected. While GOES satellite-derived winds have high temporal resolution, their spatial resolution (both horizontally and vertically) leaves much to be desired. In fact, the chances of a wind speed and direction near $700 \mathrm{mb}$ altitude over our domain is very small indeed. Wind speeds from locations outside the domain could be used; however it does not make synoptic sense to assume that wind hundreds of miles away from our domain are representative of the wind field everywhere. Synoptic weather features (such as fronts and low pressure systems) cause wind shifts that may not be present over our domain. 


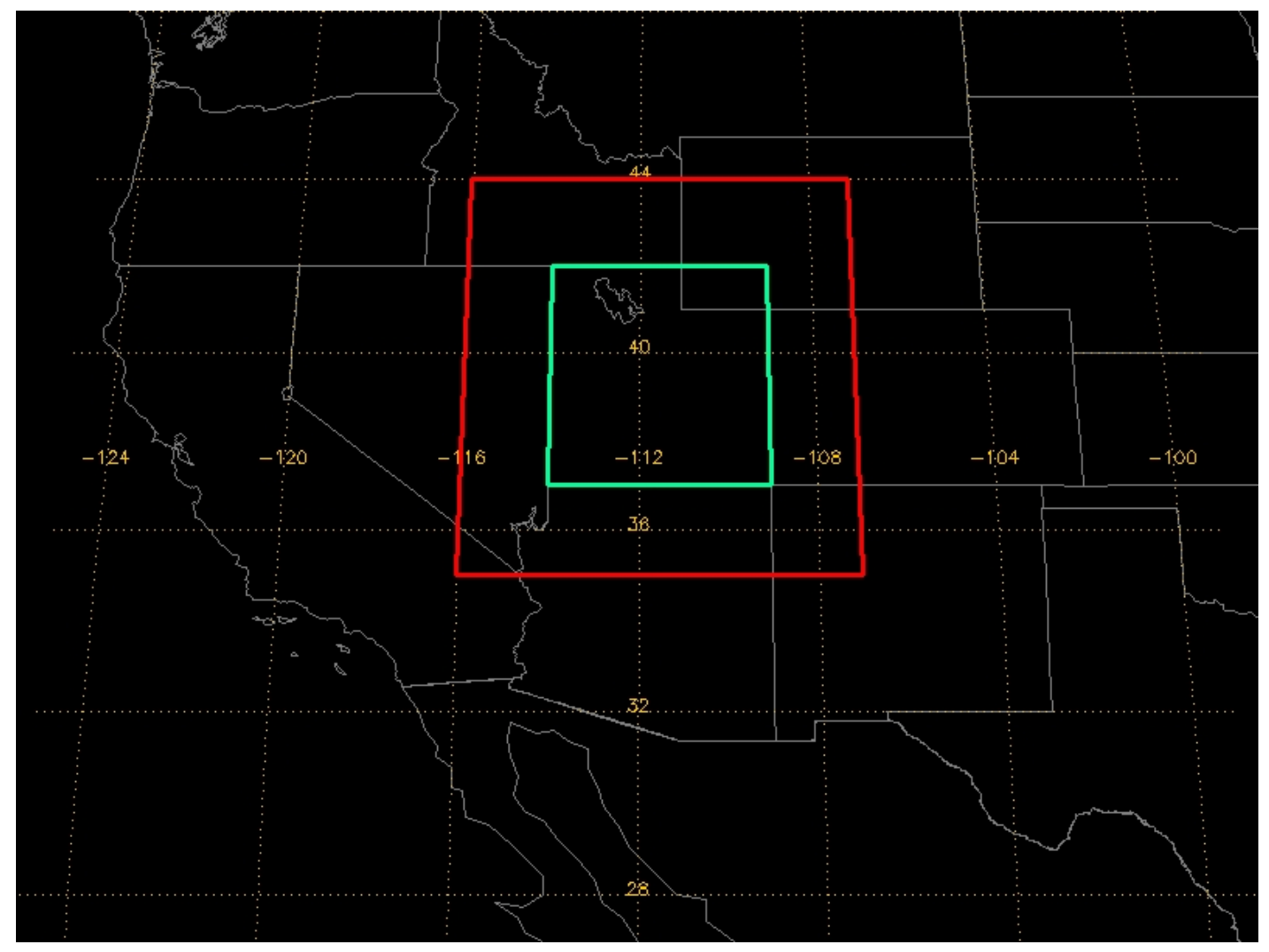

Fig 12: Map of the western United States depicting both the evaluation domain (green) and the larger displacement domain (red) to allow clouds to displace into and out of the evaluation domain.

To overcome this wind problem, weather balloon sounding data was used at a location within our domain. In Utah, that location is at the Salt Lake City National Weather Service office. Although the temporal resolution of weather soundings is four times coarser than GOES winds (only two soundings are launched every day, at 00:00 UTC and 12:00 UTC) the vertical resolution is much higher. Note that some days did not have a sounding launched within twelve hours of a Terra orbit. These days were excluded from our study in order to avoid introducing extremely inaccurate wind information. 
To yield an average $700 \mathrm{mb}$ wind, wind data between $750 \mathrm{mb}$ and $650 \mathrm{mb}$ was averaged to yield a mean wind speed and wind direction. All clouds were assumed to move at this speed and in this direction and were displaced the appropriate distance based on the amount of time between the Terra satellite pass and the Aqua satellite pass. The wind information was extracted from whichever sounding was temporally closest to the Terra pass, even if the sounding was technically in the future. For example, a 5:00 UTC Terra pass would utilize a sounding taken at 00:00 UTC on that same day, but an 18:00 UTC Terra pass would use a sounding taken at 00:00 UTC the following day.

Equation (3) describes the displacement scheme used to calculate a cloud's new latitude. Equation (4) describes how a cloud's new longitude was calculated:

$$
\begin{gathered}
l a t^{*}=\left(\frac{t^{*} y}{111000}\right)+\text { lat } \\
\text { lon }^{*}=\left(\frac{t^{*} x}{111000 * \cos (\text { lat })}\right)+\text { lon }
\end{gathered}
$$

In Equation (3), lat* is the new latitude, $t$ is the amount of time between the Terra and Aqua orbits in seconds, $y$ is the distance the cloud will travel in the y-direction in one second, 111,000 is the distance in meters in one degree of latitude, and lat is the original latitude of the cloud. In Equation (4), lon* is the new longitude, $t$ is the same as in (3), lat is the original latitude, and lon is the original longitude.

Once the new latitude and longitude positions have been calculated, the same interpolation scheme and point by point comparison as in the persistence forecast is 
performed. Of course, it is unlikely that all clouds will move at the same speed (unless they are all roughly at the same altitude). Thus, a forecast method in which clouds move at different speeds must also be analyzed. 


\subsection{Various Height Wind Displacement Method}

The Various Height Wind Displacement Method follows a similar methodology to that of the $700 \mathrm{mb}$ Wind Displacement Method, except it allows for clouds at different heights to displace at different speeds. For each cloudy pixel, a cloud-top pressure is also provided by MODIS using the $\mathrm{CO}_{2}$ slicing method described in Section 3.2, allowing each cloudy pixel to be assigned a pressure. Wind information is again extracted from the weather sounding data, this time with many average wind speeds and directions calculated at various pressure levels. This is summarized in Table 2. Note that if the cloud top pressure is below $950 \mathrm{mb}$, the cloud is persisted and not moved with the wind.

Once each cloud element is moved at its own speed, the data must be interpolated. However, the cloud data points are now randomly interspersed and are no longer at $5 \mathrm{~km}$ intervals. Simply putting clear areas between clouds on a $1 \mathrm{~km}$ grid will create too many clear areas and not enough cloudy areas. To overcome this, the original grid spacing for the Terra orbit is used through a nearest-neighbor technique (Figure 13). If there is a cloud within four kilometers of a Terra grid point, as determined by comparing numerous images to see which threshold distance best captures the observed cloud field, that grid point is labeled as cloudy. If not, that grid point is labeled as clear. Preserving this resolution of the data allows for the interpolation scheme from the previous two forecast methods to be used. In addition, there could be a situation such that a clear point and a cloudy point share the same grid box. Using the original Terra grid points also eliminates this problem since it counts only the cloudy point in that grid box (since the clear points are not displaced as the cloudy points are). 
Table 2: List of wind pressures averaged to yield a mean wind speed and direction, applied to all clouds with cloud-top pressures in that range.

\begin{tabular}{|c|}
\hline Average Wind Pressure Levels (mb) \\
\hline $950-850$ \\
\hline $850-750$ \\
\hline $750-650$ \\
\hline $650-550$ \\
\hline $550-450$ \\
\hline $450-350$ \\
\hline $350-250$ \\
\hline $250-10$ \\
\hline
\end{tabular}



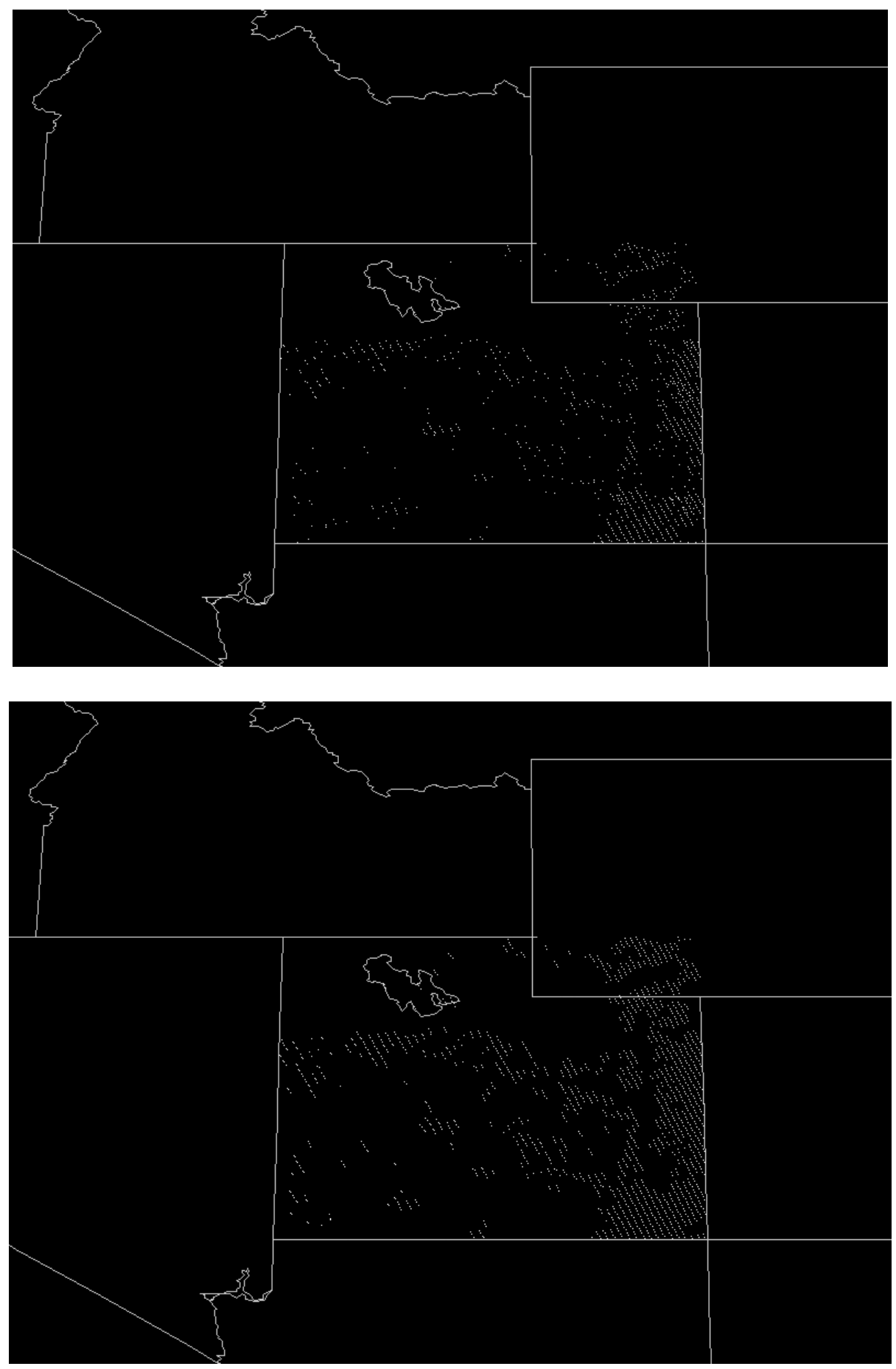

Fig 13: Unevenly dispersed data points after Various Winds Method displaces clouds at different speeds (top) and evenly displaced data points after using nearest neighbor approach on the original Terra MODIS grid-spacing (bottom) on 1 Dec 2008 at 9:20 UTC. 


\subsection{Terrain Wind Displacement Method}

To truly prove or disprove our hypothesis of high clouds displacing with the wind while low clouds persist over terrain features, a fourth forecast method introducing terrain is presented. Terrain data was imported from the United States Geological Survey Center for Earth Resources Observation and Science website. The topographical data, called GTOPO30, has terrain data for the entire planet at 30 arc-second resolution (approximately $1 \mathrm{~km}$ ). Such high resolution makes this data ideal for our study (Figure 14).

The cloud nowcast process for the Terrain Wind Displacement Method is almost exactly the same as that of the Various Wind Displacement Method, with one minor alteration. Once the cloud field has been established, it is compared to the location of complex terrain on all sides of its latitudinal and longitudinal position. That is, the cloud location is treated as the center point and the terrain elevation at the eight surrounding points are analyzed (at proximities of $1 \mathrm{~km}, 5 \mathrm{~km}$, and $15 \mathrm{~km}$ ). A minimum height of 2,500 meters (approximately 8,000 feet) is established to meet this criterion. If any of the surrounding points are higher than this threshold, the cloud at the original point is persisted. Otherwise, the cloud is displaced as in the Various Wind Displacement Method. 


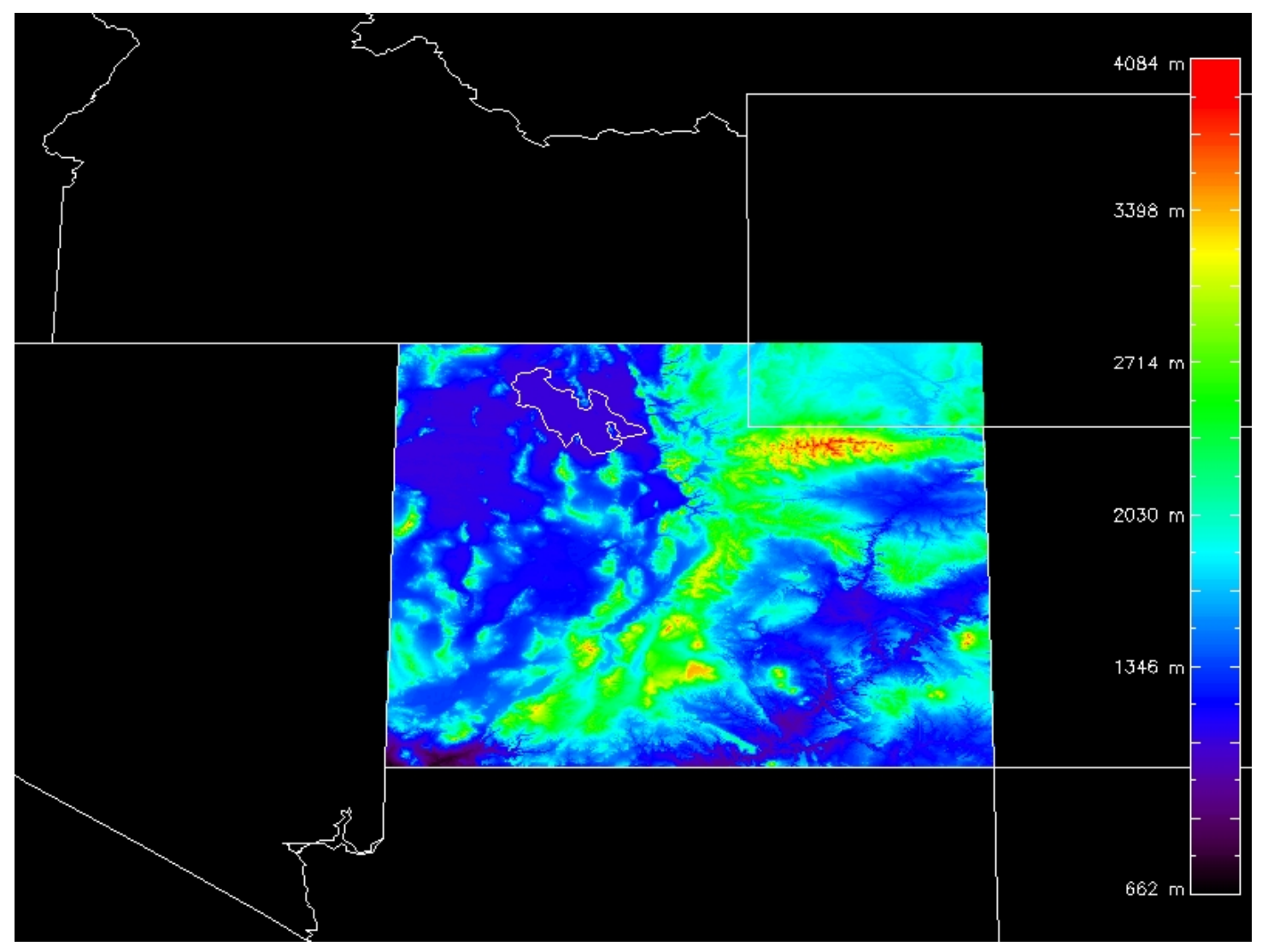

Fig 14: Terrain map of evaluation domain over Utah and SW Wyoming. 


\subsection{Cloud Climatology Method}

A cloud climatology is one of the most basic nowcasting techniques. Therefore, it is beneficial to construct one for this dataset to serve as a "standard to beat." For each

period, the percentage of cloud cover for each grid box is computed using all Aqua satellite passes combined. If it is cloudy in a specific grid box $50 \%$ of the time or more, that grid box is considered to be cloudy in the climatology forecast. If not, it is considered to be clear. 


\subsection{Cloud Phase Displacement Method}

Lastly, a nowcasting method involving cloud phase is introduced. As described in Section 3, cloud phase information (liquid water, ice, mixed, and uncertain) is extracted from the MODIS sensor as well. Utilizing this knowledge, clouds can be persisted (as in the Persistence Method) or displaced (as in the Various Wind Displacement Method) based on their phase. For example, ice clouds should be more likely to displace with the wind while liquid water clouds may be more likely to persist. 


\subsection{Temporal Domain Considerations}

The temporal domain for this study begins on 1 December 2008 and ends on 30 November 2009, with the amount of time between Terra and Aqua orbits ranging between 1.5 hours to up to 4.5 hours. After filtering all cases such that all cases populate our domain and winds did not displace all clouds out of our displacement domain, over 200 cases were left (Table 3 ).

Table 3: Number of cases by month over Utah / SW Wyoming domain.

\begin{tabular}{|c|c|}
\hline \multicolumn{2}{|c|}{ Cases over Utah / SW Wyoming } \\
\hline Month and Year & Number of Cases \\
\hline Dec-08 & 15 \\
\hline Jan-09 & 16 \\
\hline Feb-09 & 22 \\
\hline Mar-09 & 15 \\
\hline Apr-09 & 18 \\
\hline May-09 & 16 \\
\hline Jun-09 & 18 \\
\hline Jul-09 & 18 \\
\hline Aug-09 & 15 \\
\hline Sep-09 & 18 \\
\hline Oct-09 & 19 \\
\hline Nov-09 & 17 \\
\hline & \\
\hline Total & 207 \\
\hline
\end{tabular}




\subsection{Forecast Skill Evaluation}

In order to properly assess which forecast method best captures the future cloud cover, the skill of the forecast must be evaluated. Skill scores have been developed "by a desire to equalize effects of intrinsically more or less difficult forecasting situations, when comparing forecasters or forecast systems" (Wilks 2006). That is, some forecasts are more difficult to make than others, and skill scores attempt to take this fact into account.

Probabilistic forecasts are often dichotomous forecasts, such that the event occurring or the event not occurring are the only two possible forecast outcomes. Using a cloud mask algorithm to determine the presence of cloud cover is a dichotomous forecast situation (since cloud present or no cloud present are the only two possibilities), thus allowing for the use of a $2 \times 2$ contingency table. Figure (15) illustrates a typical contingency table for a dichotomous forecast. If a cloud is forecasted to occur in a particular grid box and a cloud does in fact occur there, the forecast is considered to be a hit. If a cloud is forecasted to occur and a cloud does not occur, the forecast is considered to be a false alarm. If no cloud is forecasted to occur and a cloud does occur, the forecast is considered to be a miss. Finally, if no cloud is forecasted to occur and no cloud occurs there, the forecast is considered to be a correct negative.

Many different skill scores have been derived from this simple contingency table, one of the most popular being the critical success index (Equation 5) (Gilbert 1884). The critical success index (CSI) is the number of hit forecasts divided by the sum of the hits, misses, and false alarm. It excludes the correct negative category because a correct negative event (successfully forecasting an event not happening) can be quite common 


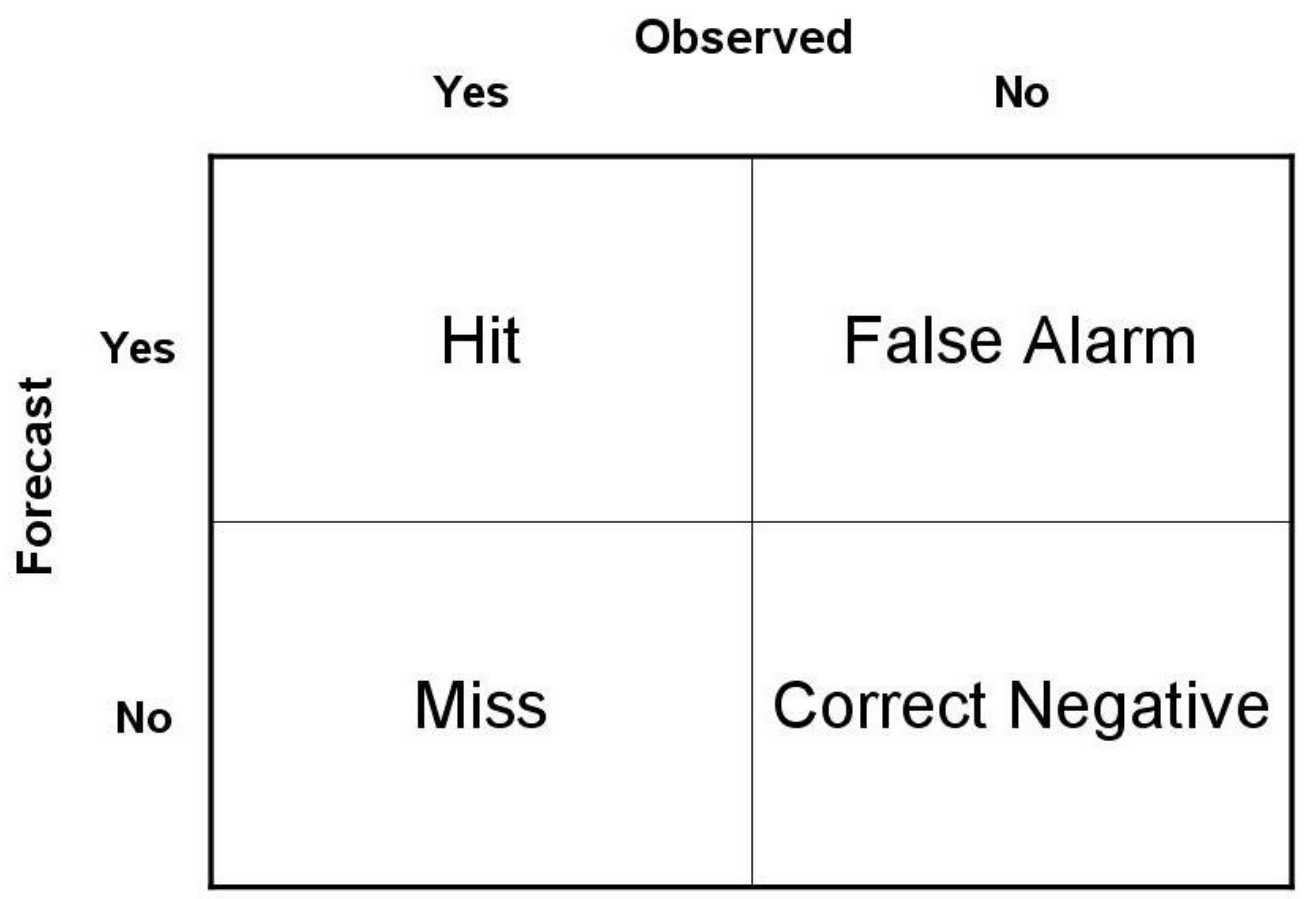

Fig 15: 2 x 2 contingency table describing all possible dichotomous forecast scenarios.

and therefore should not be included when calculating the forecast's skill (since issuing a correct negative forecast often illustrates no skill). A perfect CSI has a value of one, while a forecast with no CSI has a value of zero.

$$
\mathrm{CSI}=\frac{\text { hits }}{\text { hits }+ \text { misses }+ \text { false alarms }}
$$

The probability of detection (POD) is also a useful skill score, defined as the number of hits divided by the sum of the hits and misses (Equation 6). Essentially, the POD evaluates how well an issued forecast is at capturing where the yes events actually occur. A perfect POD value is one, while the lowest POD is zero. 


$$
\mathrm{POD}=\frac{\text { hits }}{\text { hits }+ \text { misses }}
$$

The false alarm ratio (FAR) is defined at the number of false alarms divided by the sum of the hits and false alarms (Equation 7). The FAR evaluates the fraction of how many yes events were incorrect, thus switching the orientation of its values. That is, a perfect FAR value is zero, while the lowest FAR score is one.

$$
\mathrm{FAR}=\frac{\text { false alarms }}{\text { hits }+ \text { false alarms }}
$$

The probability of false detection (POFD) is defined as the number of false alarms divided by the sum of the false alarms and correct negatives (Equation 8). The POFD mathematically contrasts the POD in that it evaluates the fraction of how many yes events were incorrect when compared to events that do not occur. A perfect POFD value is zero, while the lowest POFD score is one.

$$
\text { POFD }=\frac{\text { false alarms }}{\text { false alarms }+ \text { correct negatives }}
$$

These skill score parameters above were derived for and are primarily applied to evaluation of specific weather events occurring. Examples include the occurrence of tornados or other severe weather such as large hail or high winds. In this context, negating any importance of the correct negative forecast makes sense because it often 
demonstrates no skill. For example, one could make a forecast for Fort Collins, $\mathrm{CO}$ and say that a tornado will not occur for the next year. Based on the tornado climatology for the city, it is likely that such a forecast will in fact verify. But the question remains: does such a forecast require any skill to make?

Most people would agree that such a forecast has no skill whatsoever when being made, due to the relative low frequency of the event occurring on any given day, and as a result, the previously mentioned skill score parameters were derived to put emphasis on correctly forecasted events (i.e. a tornado was forecasted to occur today, and one actually did occur). However, should this same approach be taken towards cloud forecasts? For example, one can look out the window on a cloudy day and decide to forecast that the sky will still be cloudy in a few hours. On the other hand, one can look out the window on a mostly clear day and decide to forecast that the sky will remain mostly clear in a few hours. Now assume that both forecasts verify correctly (the sky remained cloudy on the cloudy day and clear on the clear day). Both forecasts required skill to be made accurately due to the dynamic nature of the clouds themselves. Clouds (unlike tornadoes) are high-frequency events, dissipating and reforming on a regular basis. Knowledge of the synoptic meteorological situation is required to make accurate cloud forecasts.

By Equations (5) - (8), only the correct-cloudy forecasts are evaluated as skillful, while the correct-clear forecasts are given no weight in terms of skill. For this reason, we propose new skill scores which weight both hit and correct negative forecasts equally in skill evaluation. Figure (16) describes a modified $2 \times 2$ contingency table, keeping the same definitions of a hit, miss, false alarm, and correct negative as in Figure (15), but treating a correct negative forecast as a correct yes forecast. As a result, the forecast 


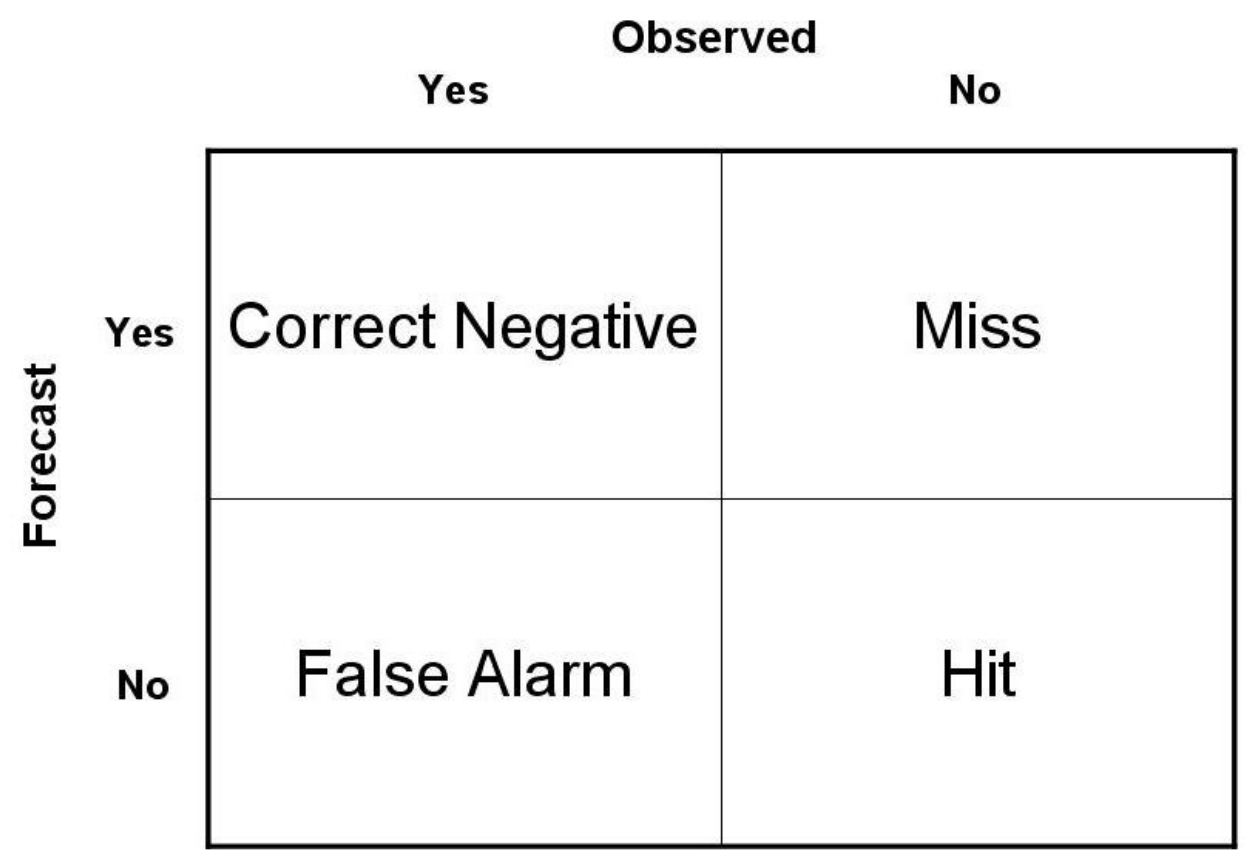

Fig 16: Modified 2 x 2 contingency table, assuming that a correct negative is a correct yes forecast. The other category locations have been modified accordingly.

categories switch places with each other across the diagonal of the table. For example, if a correct negative is considered a hit (that is, clear areas are the goal of this forecast) then an area predicted to be clear but actually cloudy is a false alarm, which is the same as a miss if we retain the original definition of a yes/yes cloudy forecast as a hit.. In the same way, miss forecasts become false alarms using the original definition, and hit forecasts become correct negatives.

Extending this idea to Equations $(5)-(8)$, we define new skill score parameters that take into account correct clear areas.

$$
\text { Clear CSI }=\frac{\text { correct negatives }}{\text { correct negatives }+ \text { false alarms }+ \text { misses }}
$$




$$
\text { Clear POD }=\frac{\text { correct negatives }}{\text { correct negatives }+ \text { false alarms }}
$$

$$
\text { Clear FAR }=\frac{\text { misses }}{\text { correct neagties }+ \text { misses }}
$$

$$
\text { Clear POFD }=\frac{\text { misses }}{\text { misses }+ \text { hits }}
$$

Equations (9) - (12) are our "clear" skill score parameters that account for areas that were accurately forecasted as clear. These parameters also range from zero to one and exhibit behavior exactly like their counterparts (i.e. the Clear CSI's best skill score is also one, while the worst is zero).

Using two different skill scores to diagnose the same forecast can be confusing, thus it is beneficial to combine Equations (5) - (8) with Equations (9) - (12). This is easily achieved by simply adding Equation (5) to Equation (9) (and so forth with the other equation pairs) and dividing by two (to preserve the equation's behavior between zero and one). Thus, we yield:

Total CSI $=\frac{\frac{\text { hits }}{\text { hits }+ \text { misses }+ \text { false alarms }}+\frac{\text { correct negatives }}{\text { correct negatives }+ \text { false alarms }+ \text { misses }}}{2}$

$$
\text { Total POD }=\frac{\frac{\text { hits }}{\text { hits }+ \text { misses }}+\frac{\text { correct negatives }}{\text { correct negatives }+ \text { false alarms }}}{2}
$$




$$
\text { Total FAR }=\frac{\frac{\text { false alarms }}{\text { hits }+ \text { false alarms }}+\frac{\text { misses }}{\text { corect negatives }+ \text { misses }}}{2}
$$

$$
\text { Total POFD }=\frac{\frac{\text { false alarms }}{\text { false alarms }+ \text { correct negatives }}+\frac{\text { misses }}{\text { misses }+ \text { hits }}}{2}
$$

Mathematically, these skill score parameters will be at their maximum values on partly cloudy days such that there is roughly the same number of hits (correct cloud areas) as there are correct negatives (correct clear areas). If a scene is forecasted to be all clear or all cloudy and verifies, the maximum value the CSI can attain is 0.5 . For this reason, we define skill scores above 0.5 to be considered "skillful," while those below 0.5 to show little skill. Figure (17) illustrates the behavior of the "cloudy" CSI, the "clear" CSI, and the "total" CSI as defined by Equations (5), (9), and (13) respectively by keeping the number of misses and false alarms constant and varying the number of hits and correct negatives. One can see that the "total" CSI is at a maximum when the "cloudy" CSI and the "clear" CSI are exactly the same, denoting the same number of correct cloudy point as correct clear points. From this point forward, when referring to CSI, POD, FAR, and POFD, our "total" definitions as described by Equations (13) - (16) will be the equations in question.

Two final useful skill scores are the percentage of forecasts correct (PFC) and the true skill statistic (TSS). The PFC (Equation (17), Finley 1884) is useful because it weights hits and correct negatives equally. The TSS (Equation (18), Flueck 1987) is unique because it varies from negative one to one, with positive numbers representing 
CSI Behavior

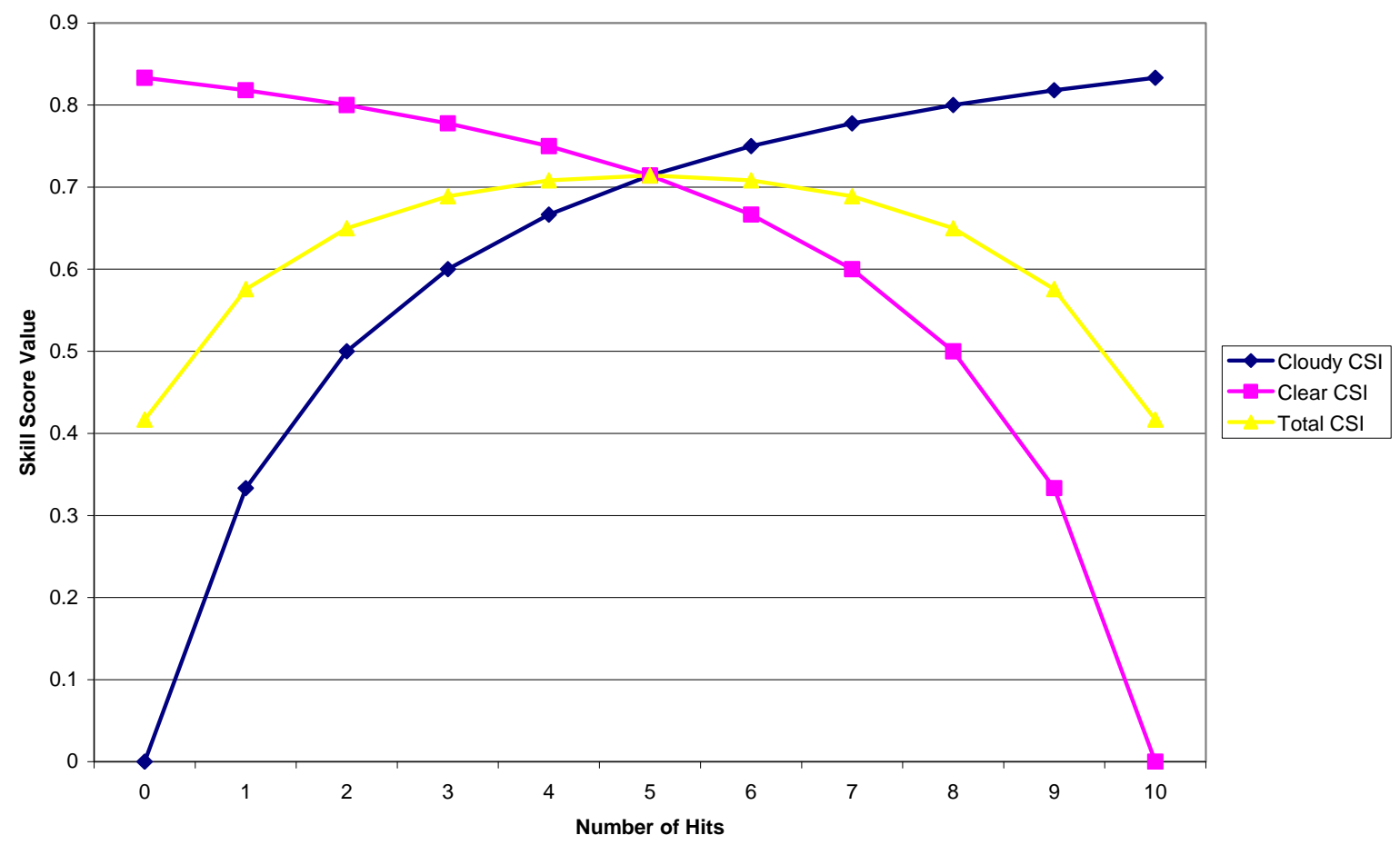

Fig 17: Behavior of "cloudy" CSI, "clear" CSI, and "total" CSI when varying the number of hits and correct negatives, but keeping misses and false alarms constant. The maximum value for "total" CSI occurs when the number of hits equals the number of correct negatives.

skill better than random guessing, zero signifying that random guessing is just as good as the forecast method in question, and negative numbers meaning random guessing is actually better than the forecast method in question. Changing the PFC or the TSS in the same way as the CSI, POD, etc. yields the same equations, thus such changes are not necessary. 


$$
\begin{aligned}
& \text { PFC }=\frac{\text { hits }+ \text { correct negatives }}{\text { hits }+ \text { misses }+ \text { false alarms }+ \text { correct negatives }} \\
& \mathrm{TSS}=\frac{(\text { hits } * \text { correct negatives })-(\text { misses } * \text { false alarms })}{(\text { hits }+ \text { misses }) *(\text { false alarms }+ \text { correct negatives })}
\end{aligned}
$$

Using Equations (13) - (18), we can evaluate the skill of our various cloud nowcasting methods. 


\section{RESULTS}

\subsection{Seasonal Skill Scores}

Skill scores for the Utah domain were calculated for three month intervals (December/January/February, March/April/May, June/July/August, and September/October/November) for all nowcast methods. Figure (18) shows CSI, POD, FAR, and POFD values for the months of Dec/Jan/Feb over the Utah domain. Persistence nowcasts performed the best, scoring higher CSI and POD scores $(\sim 10 \%$ higher than the $700 \mathrm{mb}$ and Various Winds Methods), as well as the lowest FAR and POFD values (also by about 10\%). Because the Persistence Method performs best, it is not surprising that the terrain nowcasting methods perform increasingly better as more cloud is persisted (from $1 \mathrm{~km}$ proximity to significant terrain to $15 \mathrm{~km}$ proximity). That is, as a larger amount of cloud is persisted, the Persistence Method values are approached until eventually it is recreated (all cloud is persisted).

Surprisingly, the difference between the $700 \mathrm{mb}$ Displacement method and the Various Winds Displacement method are quite small, implying their skills are similar over this region during the winter months. Random initial cloud and clear values were also processed to serve as comparison to the other nowcasting methods. That is, if any of the nowcasting methods performed less skillful than the random forecast, something might be wrong with the method itself. However, random nowcasts performed the least skillful of all.

The Climatology Method skill scores demonstrate the need for nowcasting methods. This three month period shows that although climatology does demonstrate 


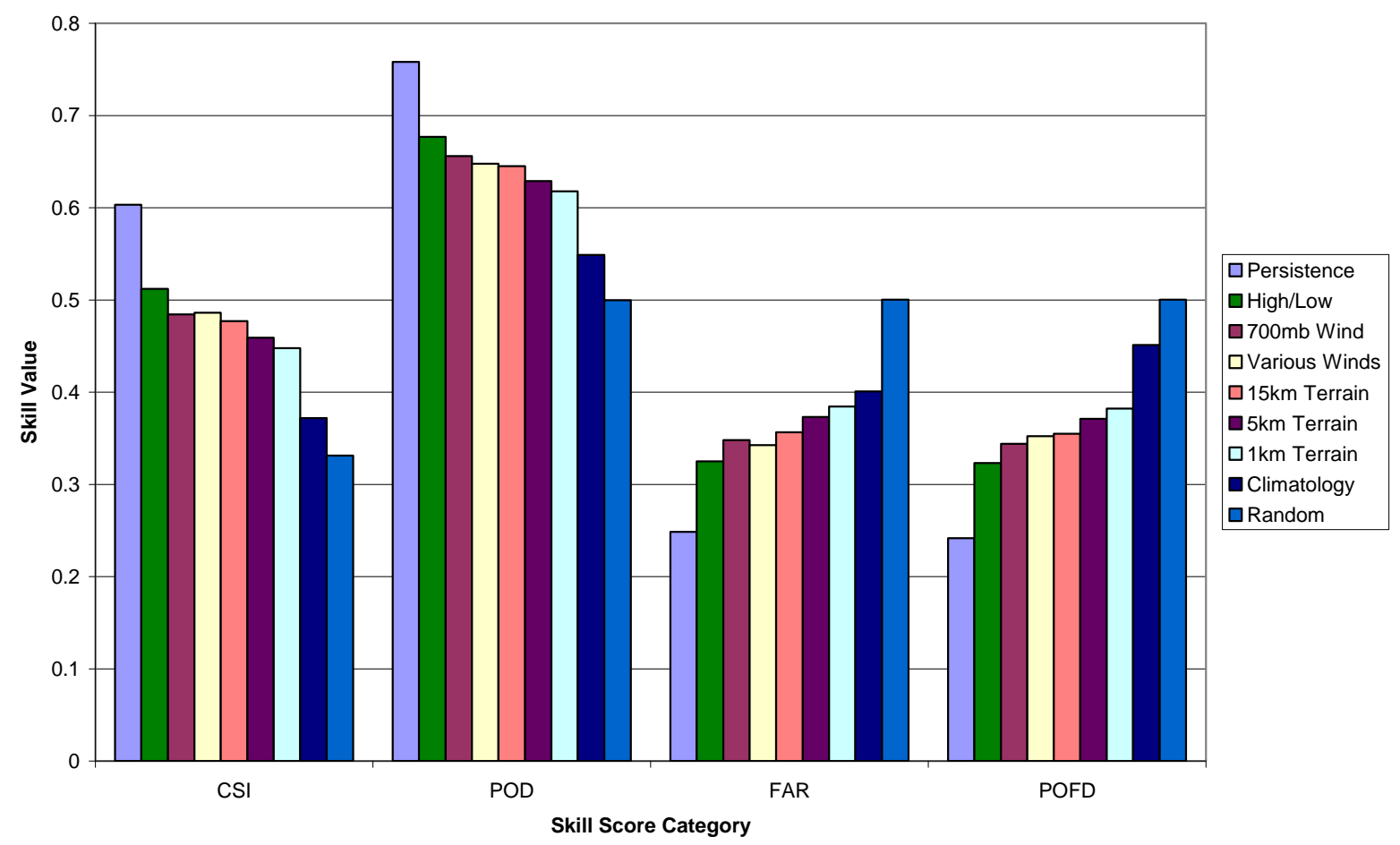

Fig 18: Skill scores over Utah from December 2008 to February 2009.

some skill over random guessing, it cannot compare to the skill for our other methods.

Climatologies are often used as a first "best guess" for the cloud nowcasting problem, thus it makes sense that knowledge of cloud presence on a particular day is a better nowcast than a three-month average.

A High/Low Method was also introduced as a last-minute data process comparison. All cloud determined to have a cloud-top pressure higher than 500mb would be displaced with the wind, while cloud lower than $500 \mathrm{mb}$ would be persisted, regardless of terrain proximity. This nowcast method performed better than all of the other methods (except for the Persistence Method) during the winter months. 


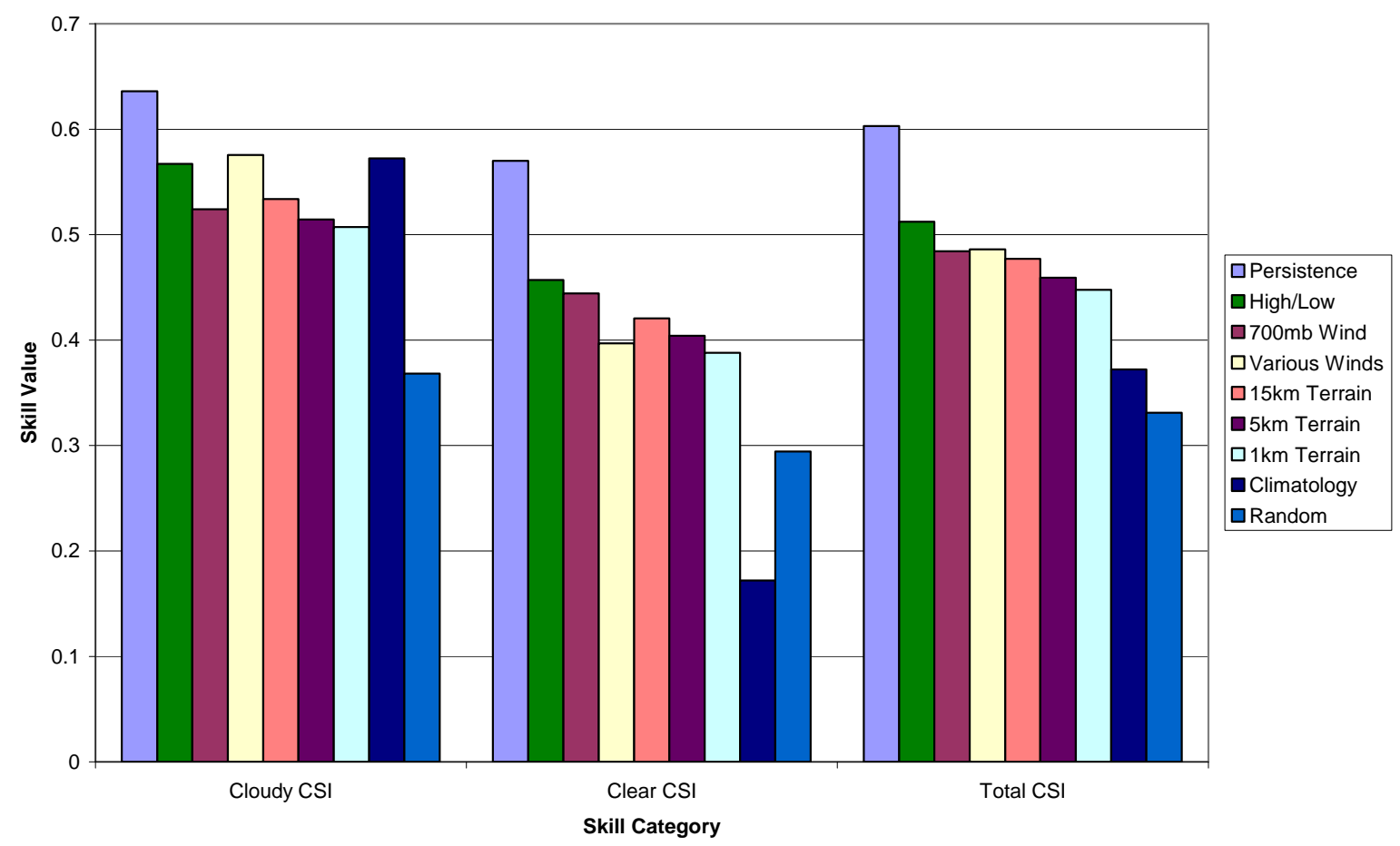

Fig 19: Cloudy, clear, and total CSI scores for various nowcast methods from December 2008 to February 2009, demonstrating the need to define these scores and combine them into a new "total" CSI.

Decomposing the CSI into its "cloudy" and "clear" values is beneficial in this case to further illustrate why our new skill scores are superior to the conventional ones (Figure 19). If only the "cloudy" scores were used (as is often the case in the literature), one might be mislead and think that the Various Wind Displacement Method performs better than the 700mb Wind Displacement Method. However, the "clear" scores show that the $700 \mathrm{mb}$ Method demonstrates more skill capturing the clear areas than the Various Method. As a result, the two even each other out and perform about the same using our skill scores. 


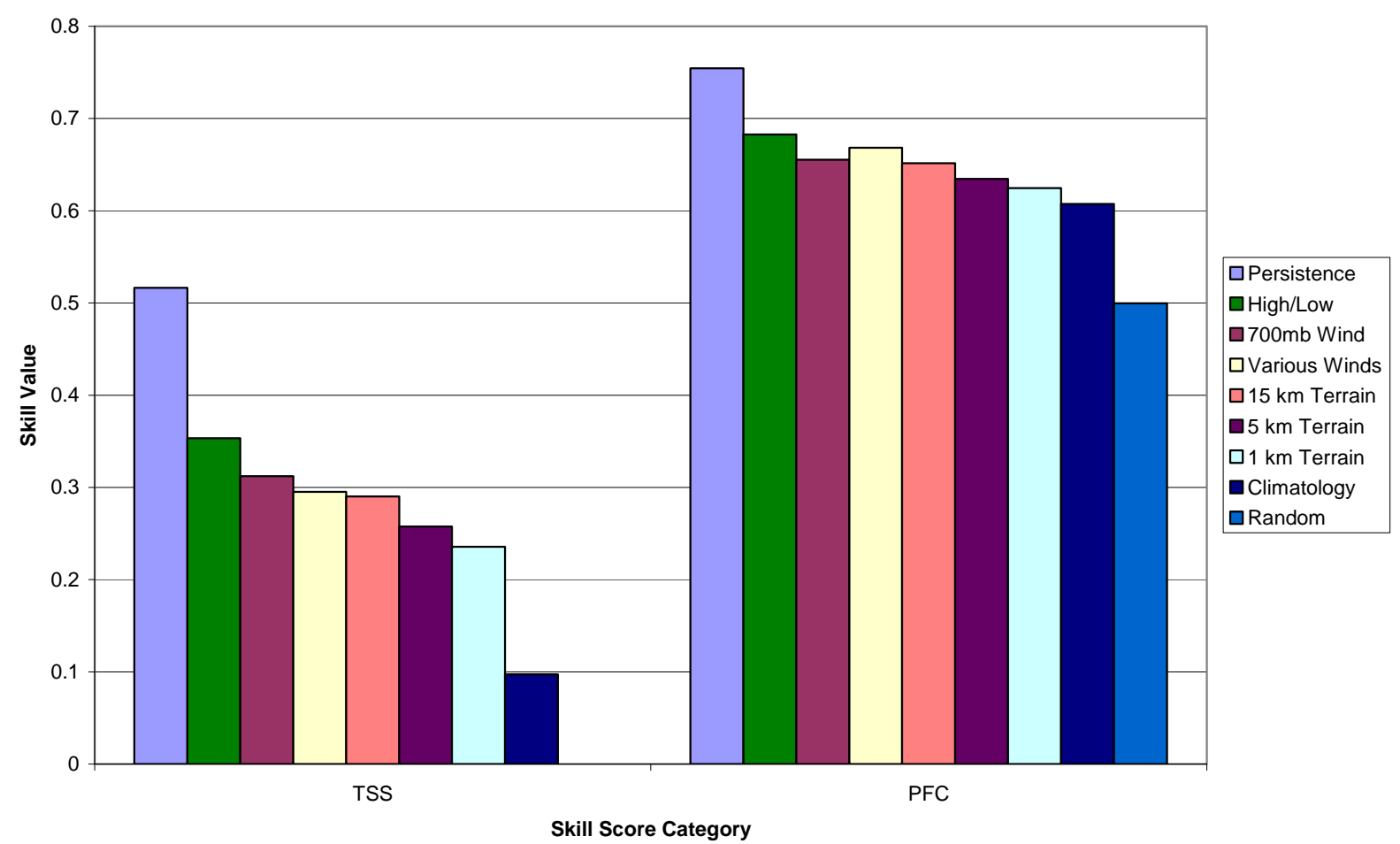

Fig 20: True Skill Statistic and Percentage of Forecasts Correct values for various nowcast methods from December 2008 to February 2009.

Figure (20) displays our final two skill scores, the TSS and the PFC. Both display similar results such that the Persistence Method demonstrates the most skill (by 20\% TSS over the $700 \mathrm{mb}$ and Various Winds Methods) and also gets the greatest number of nowcasts correct. The TSS for our Random nowcasts is zero, as it should be since a zero value means the nowcast method in question performs just as well as random guessing.

Figure (21) displays skill scores over Utah from 1 March 2009 to 31 May 2009. Again, the Persistence Method demonstrates the most skill; however the 700mb Wind Method is a close second, losing to Persistence by only 4\% CSI. During the spring months over Utah, there is a much greater difference in skill between the $700 \mathrm{mb}$ Wind 


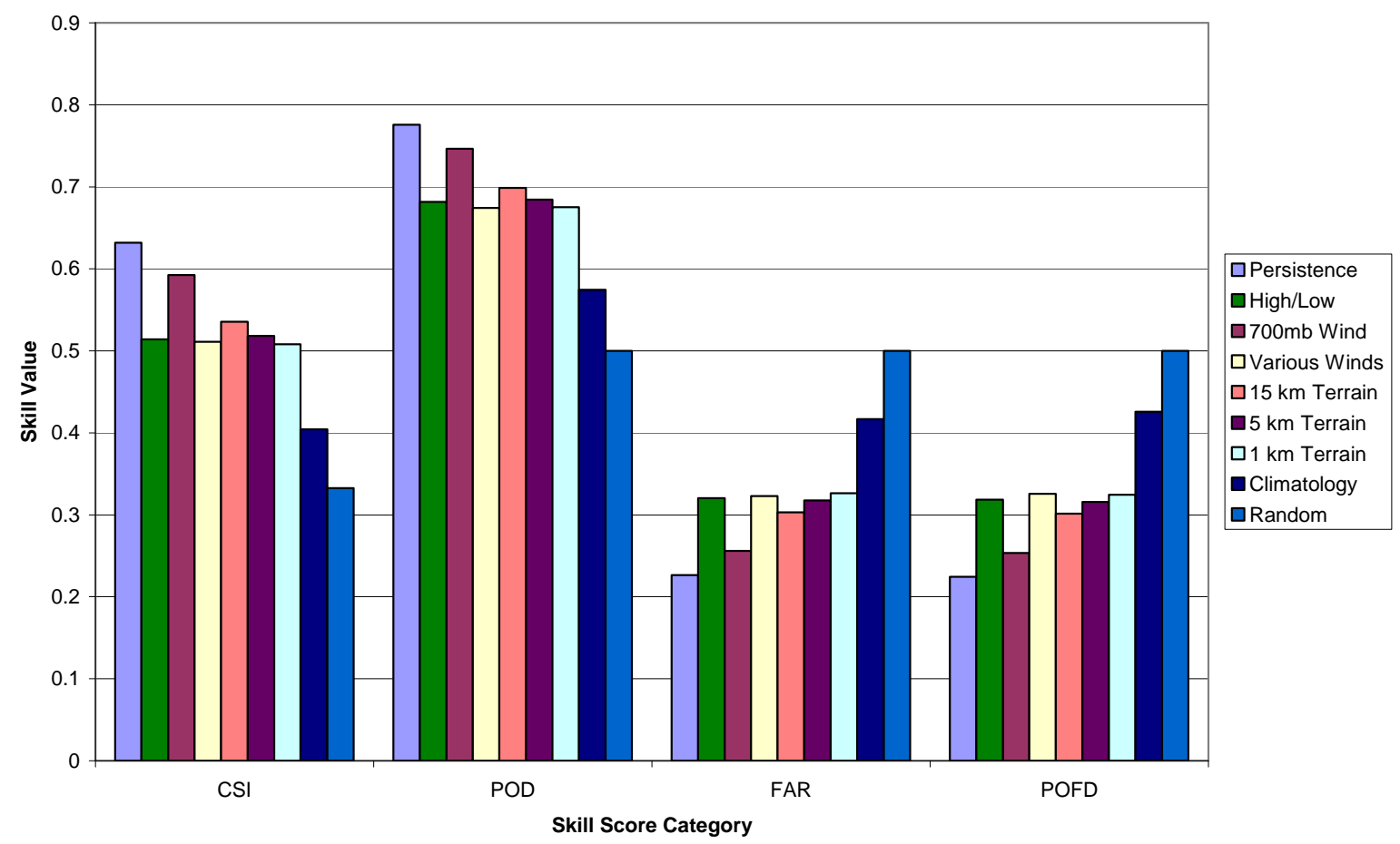

Fig 21: Skill scores over Utah from March to May 2009.

Method and the Various Wind Method than during the winter (nearly a 10\% CSI difference). The Persistence Method has the lowest FAR and POFD scores, with the $700 \mathrm{mb}$ Wind Method performing almost equally well. The $1 \mathrm{~km}, 5 \mathrm{~km}$, and $15 \mathrm{~km}$ Terrain Methods perform progressively better once again since they approach Persistence as more clouds are kept from moving. The Low/High Method performed about the same as the Various Wind Method, implying that most of the clouds present were high up and the atmosphere, allowing them to be displaced.

Figure (22) displays the TSS and PFC scores from March to May 2009 over Utah. The Persistence Method and the 700mb Wind Method differ in TSS by only 5\% for the spring months, while the Various Winds Method is nearly 15\% lower in TSS skill than the 


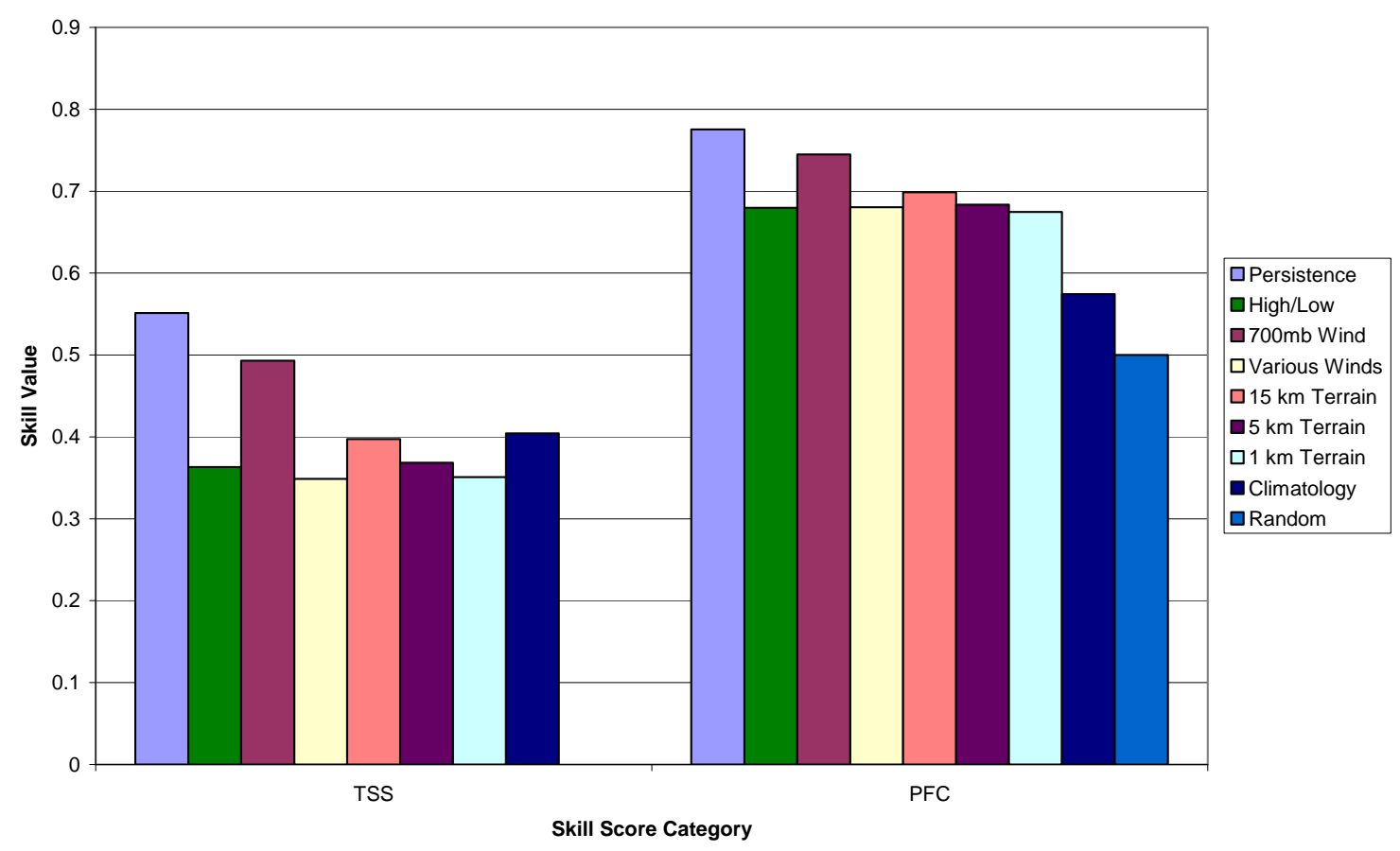

Fig 22: True Skill Statistic and Percentage of Forecasts Correct for various nowcasting methods from March to May 2009.

Persistence Method. Again, the Climatology Method exhibits more skill than random guessing; however our all methods all improve over it.

Figure (23) shows skill scores for the summer months over Utah and shows results very different from the other two seasons. The Persistence Method and the $700 \mathrm{mb}$ Wind Method demonstrate essentially the same skill as far as CSI, POD, FAR, and POFD, with all of the other methods quite close as well (worse by only approximately 4\%). This implies that during the summer, clouds are lower in the sky and thus their movement is influenced by lighter winds lower in the troposphere, causing them to not 


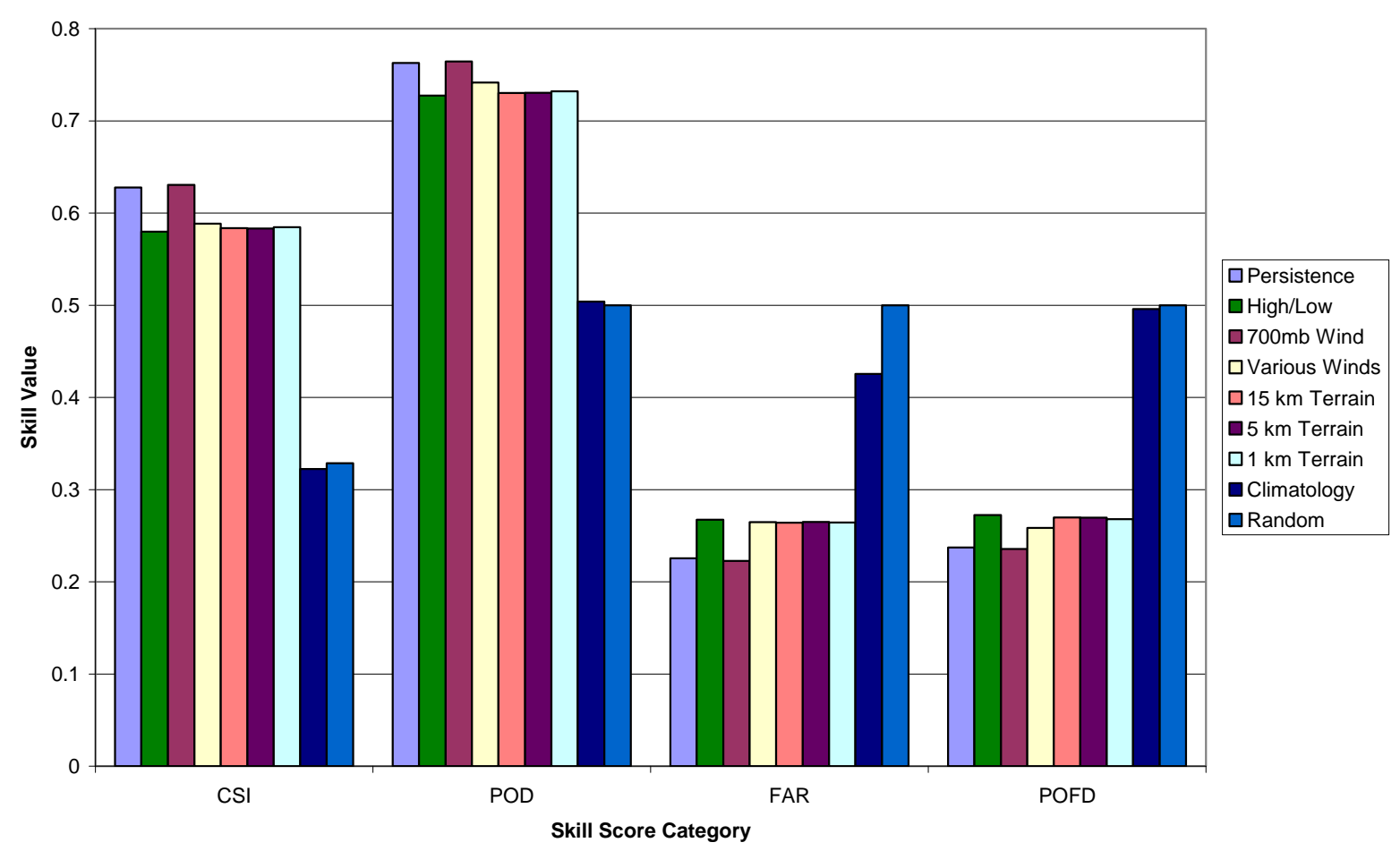

Fig 23: Skill scores over Utah from June to August 2009.

move as quickly. Since cloud movement is so slight, persistence values are essentially duplicated, causing the all of the skill scores to be quite similar.

Figure (24) shows TSS and PFC values from June to August 2009. Again, the Persistence and 700mb Methods are essentially the same, with all of the other nowcast methods about the same ( $\sim 5 \%$ less than the Persistence and $700 \mathrm{mb}$ Methods). Random nowcasts again do poorly, but that is to be expected with a methodology that exhibits no skill. Interestingly, the Climatology Method performs about the same as random nowcasts, implying that the convective nature of the summer months cause climatologies to be mainly ineffective when forecasting cloud cover. 


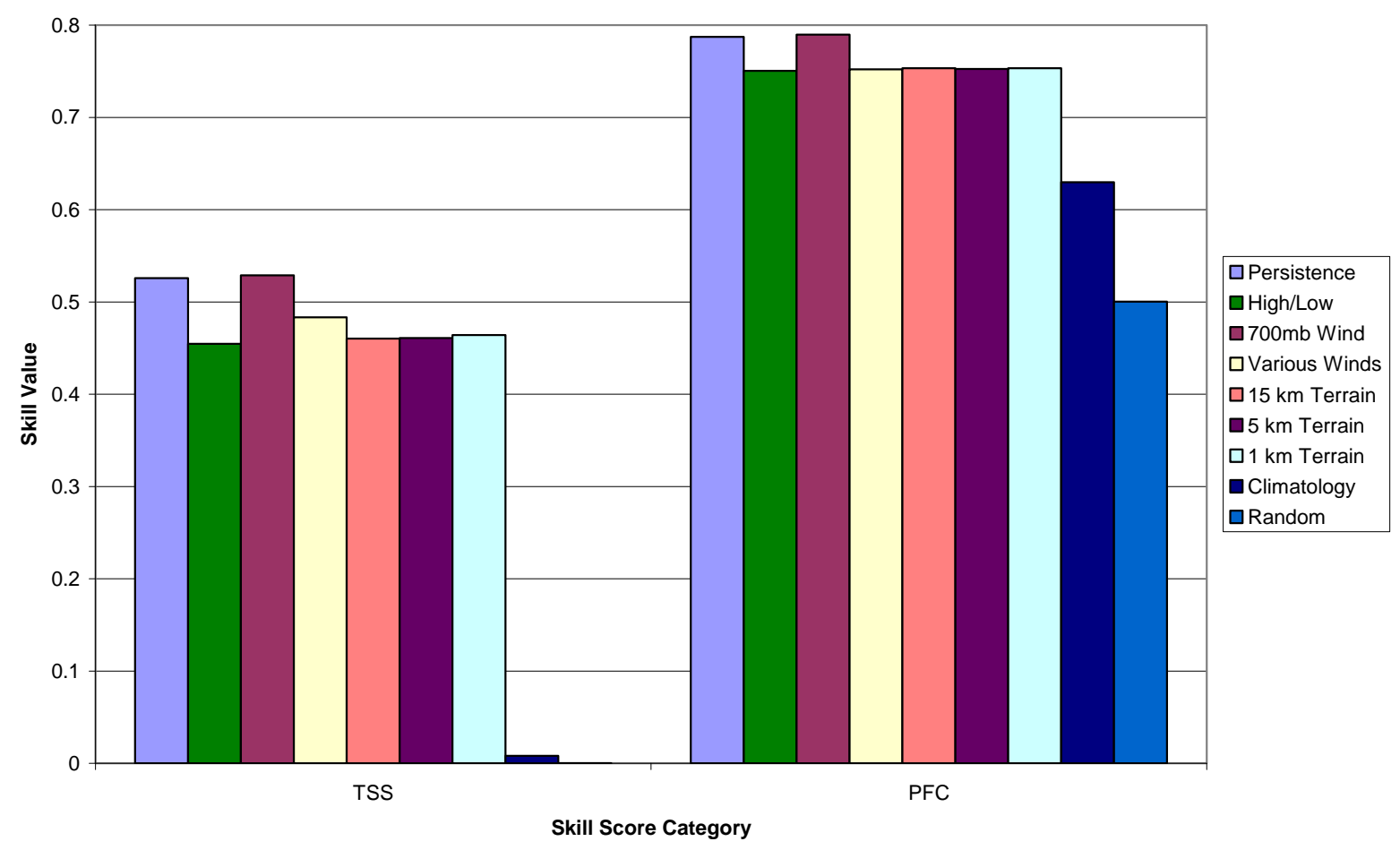

Fig 24: True Skill Statistic and Percentage of Forecasts Correct for various nowcasting methods from June to August 2009.

Figure (25) displays skill scores for the autumn months over Utah. The Persistence Method again performs the best, with the 700mb Wind and Various Winds Methods a close second ( $\sim 6 \%$ CSI less than the Persistence Method). The different Terrain Methods again get better and better as the Persistence Method is approached, with the Low/High method performing about the same as the Various Winds Method ( $\sim 1 \%$ CSI less). Since the Climatology Method is essentially as good as random guessing, its TSS score should be nearly zero just as the random nowcasts are. 


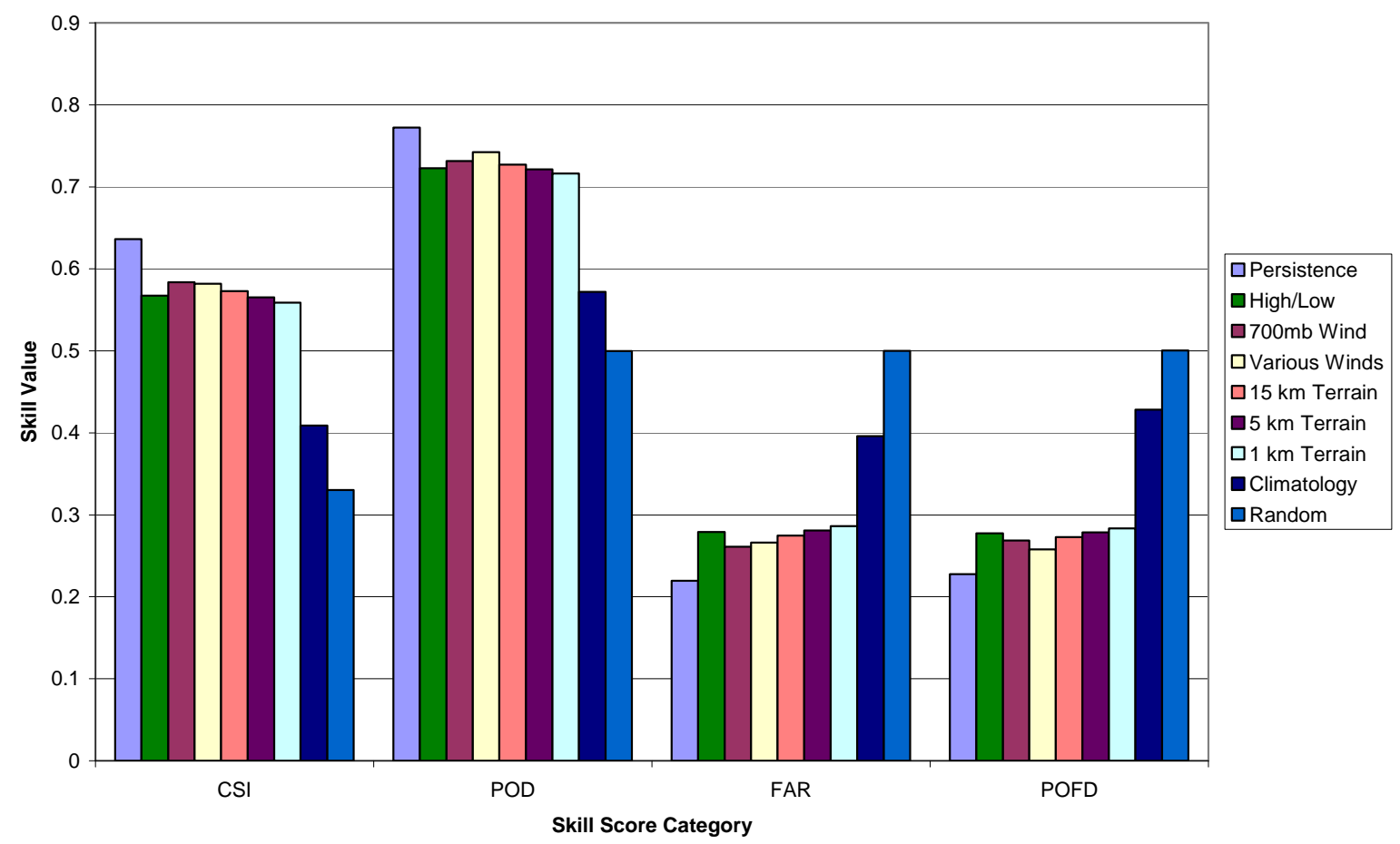

Fig 25: Skill scores over Utah from September to November 2009.

Figure (26) shows the TSS and PFC values for the autumn months over Utah.

The 700mb Method and the Various Winds Method performed about the same as far as TSS skill is concerned, but both were again beaten by the Persistence Method (by $\sim 6 \%$ TSS score). In all four seasonal breakdowns, it appears that the Persistence Method (by varying degrees of success) performs the best over the state of Utah and southwest Wyoming regardless of season. The Climatology Method again shows skill over random guessing, however all other methods exhibit more skill. 
Skill Scores over Utah from 1 September 2009 to November 302009

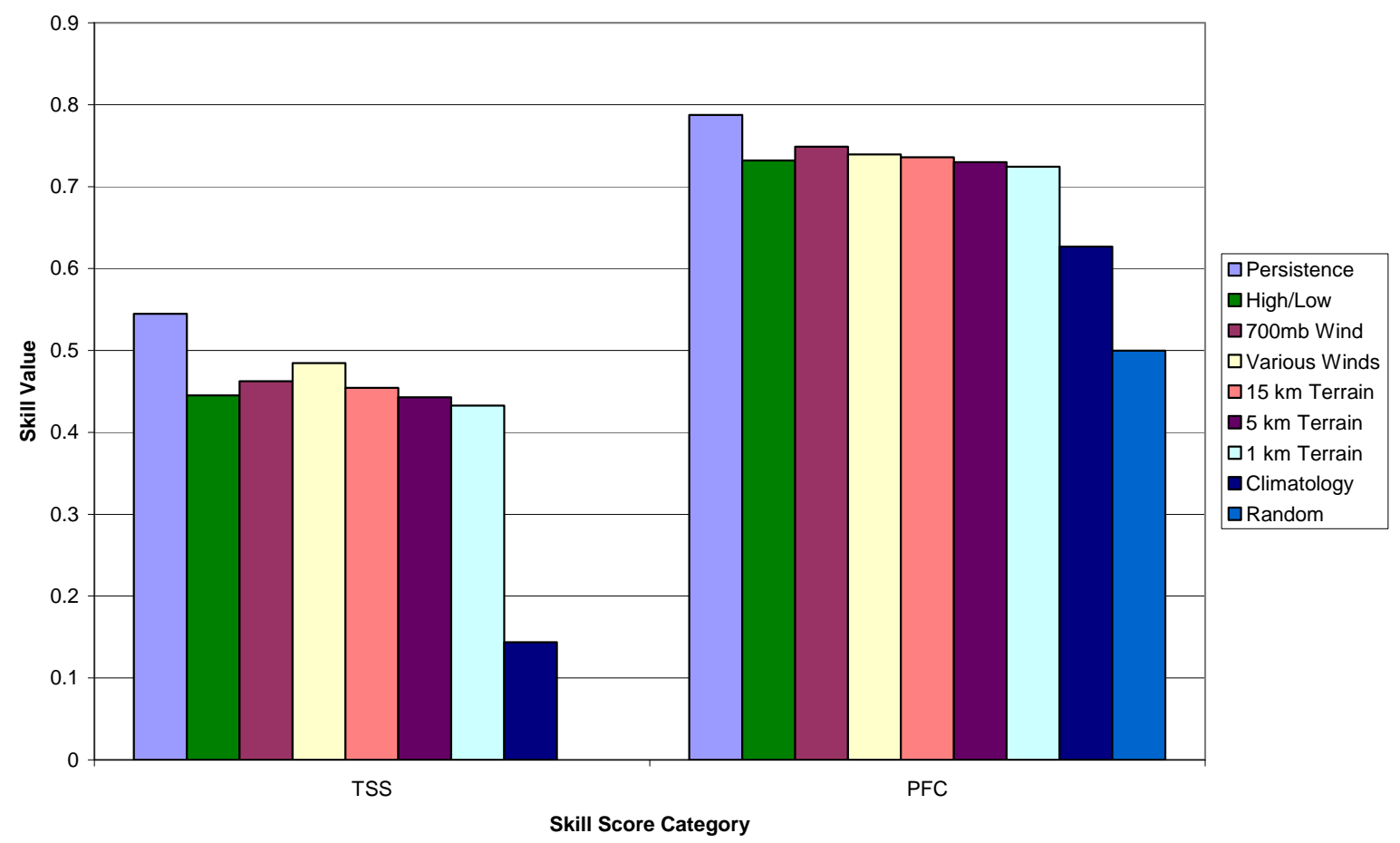

Fig 26: True Skill Statistic and Percentage of Forecasts Correct for various nowcasting methods from September to November 2009. 


\subsection{Seasonal Skill Score Variations}

It is beneficial to divide these forecast methods by season to see at which time of year the best results can be obtained. The Terrain Methods, Random Method, and High/Low Method have been excluded since their patterns have been well-established in the previous findings and variations of the Persistence and Various Winds methods (except for the Random Method). It is interesting to note that the Persistence Method performs essentially the same across all four seasons over Utah, with the largest difference occurring between the winter months and the spring/autumn months (Figure $27)$; even so, the difference itself is quite small ( $\sim 3 \% \mathrm{CSI})$.

The 700mb Wind Method (Figure 28) performs best during the summer months and does most poorly during the winter ( $\sim 15 \%$ CSI difference). This is most likely due to large synoptic cloud shields present during the winter months that fill in over time. This displacement technique does not account for cloud-filling, which does not happen as often during the summer months.

Figure (29) shows CSI skill scores for the Various Winds Method for the entire year over Utah. Unlike the 700mb Wind Method, which shows about even CSI values during the spring and autumn, the spring CSI score for the Various Winds Method is significantly lower than the autumn skill score (by $\sim 7 \% \mathrm{CSI}$ ). The best skill scores by this method also occur during the summer months, due to the more discrete nature and movement of individual clouds. 
CSI Scores for Persistence Nowcast for Utah from Dec 2008 to Nov 2009

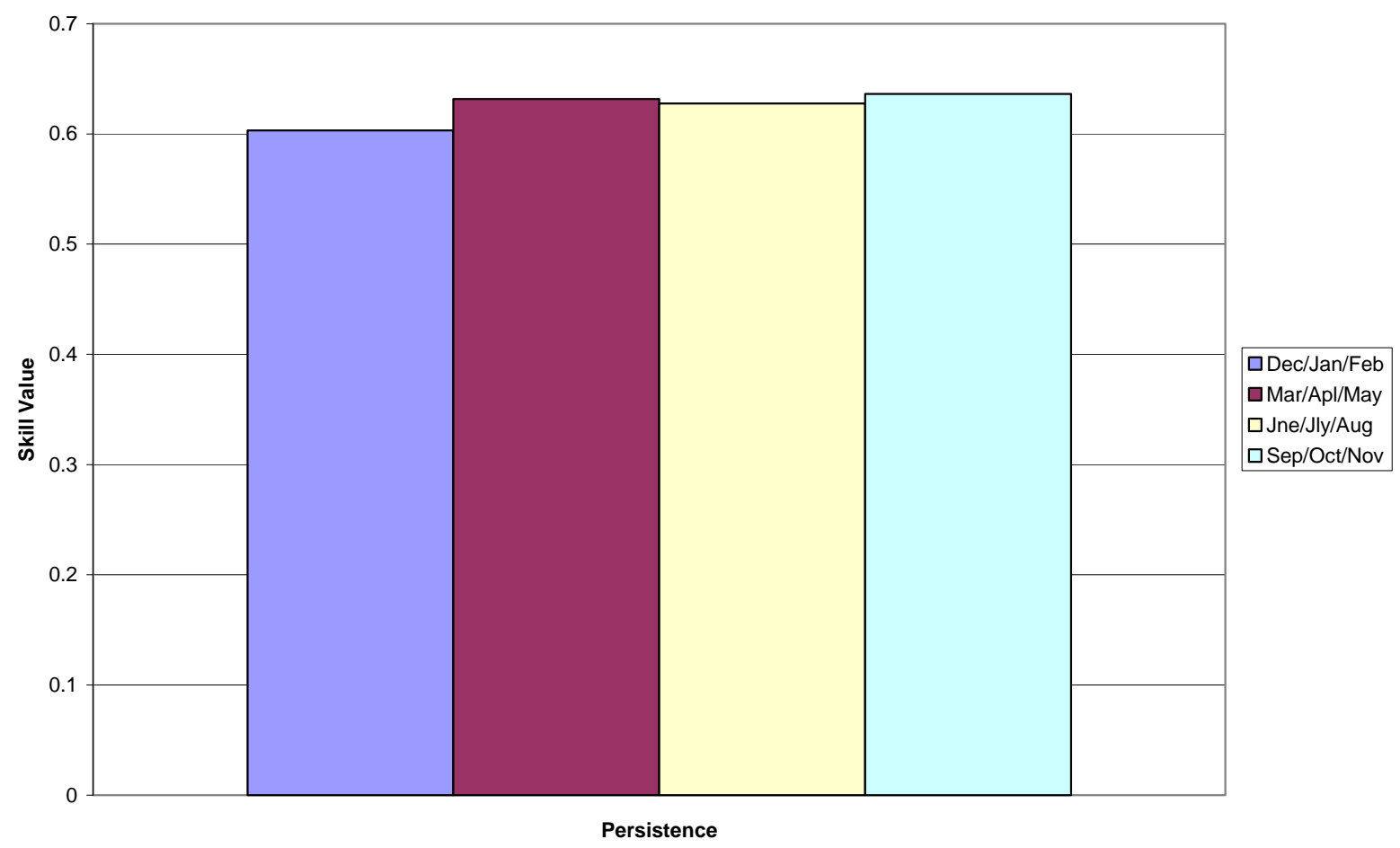

Fig 27: Graph of Persistence Nowcast Method for all four seasons over Utah. 
CSI Scores for 700mb Winds Nowcast for Utah from Dec 2008 to Nov 2009

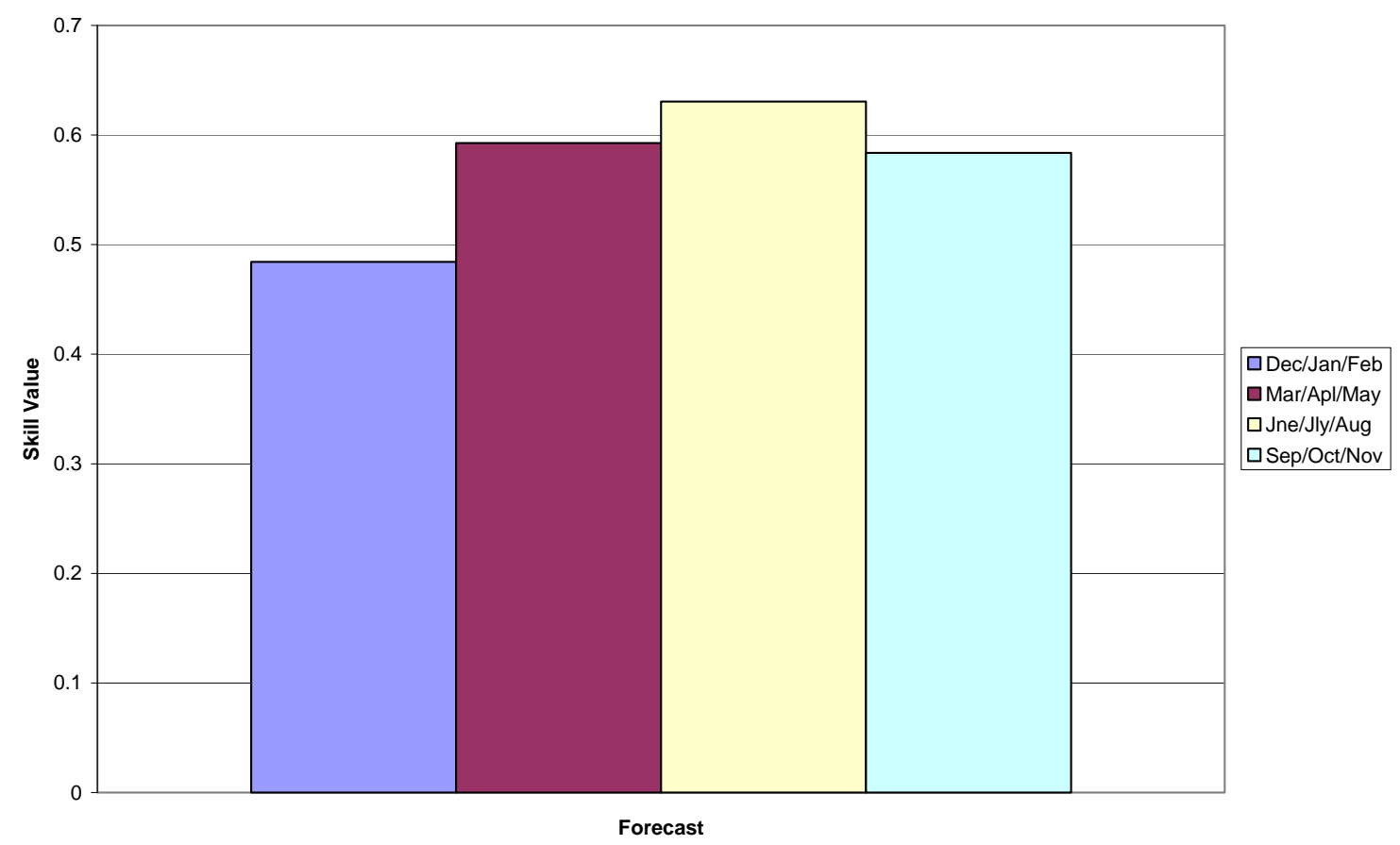

Fig 28: Graph of 700mb Wind Nowcast Method for all four seasons over Utah. 
CSI Scores for Various Winds Nowcast for Utah from Dec 2008 to Nov 2009

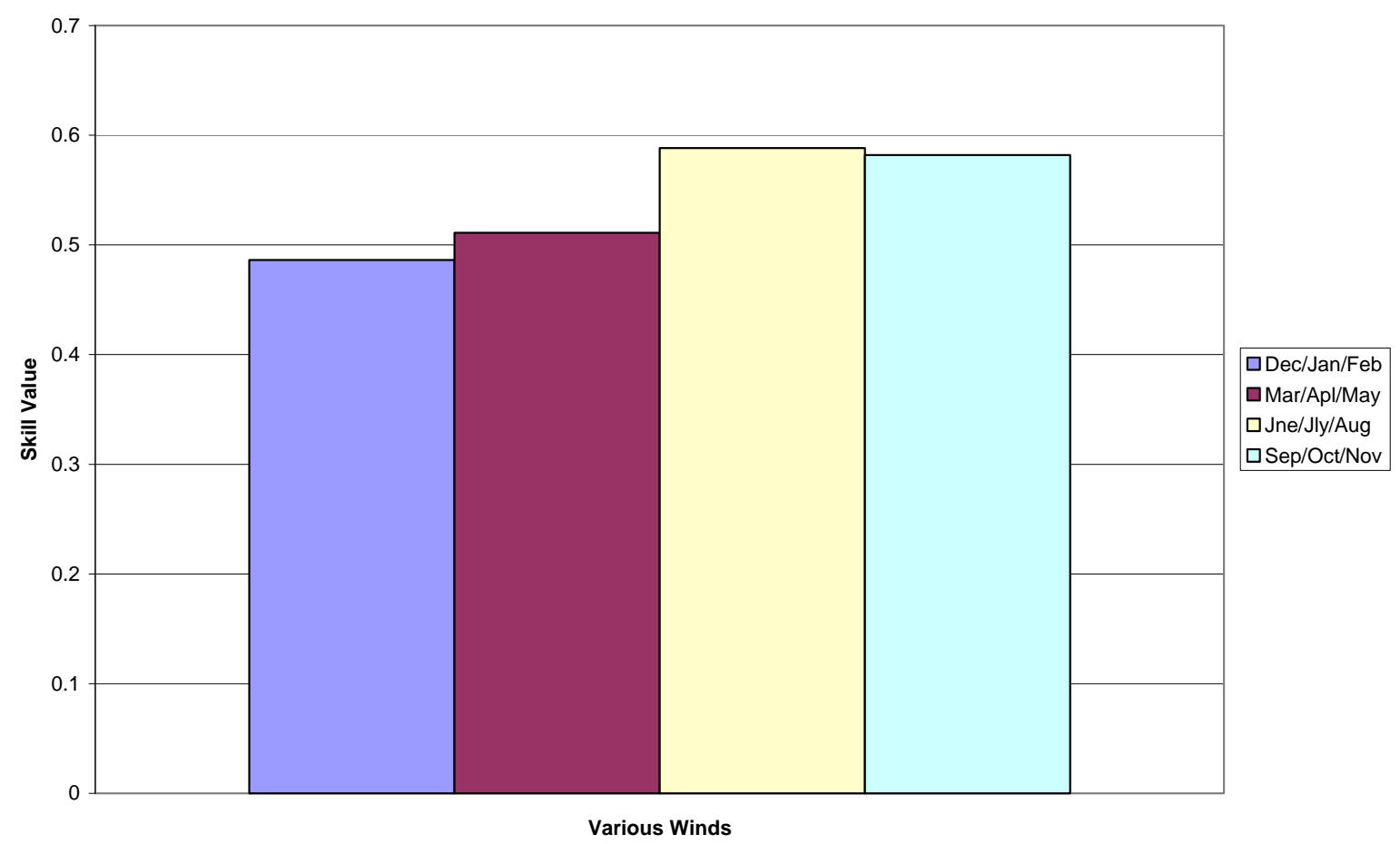

Fig 29: Graph of Various Winds Nowcast Method for all four seasons over Utah. 


\subsection{Temporal Skill Score Variations}

Dividing each nowcasting method not only by season and method, but by length of time of the nowcast is also beneficial. Figure (30) shows how temporal differences in the orbits of both Terra and Aqua affect the calculation of skill scores over Utah. Because of the orbital patterns of the two satellites, during the daytime hours, 2.5 hours or less passes between samplings of Utah. During the nighttime hours, between four and five hours passes between samplings of Utah. This graph shows that, asides from Persistence Method nowcasts during the winter months, all nowcast methods perform more poorly as the amount of time between satellite passes increases when compared to their short-term counterparts. This makes sense since the closer a sampling is to the actual time in question, the less time the clouds have to move and a better nowcast will result.

It is also interesting to note that most of the variation in these nowcasting methods is due to the nowcasts that are made in the four to five hour timeframe. That is, each method does very similar to each other regardless of time of year in the shorter timeframe and diverges as time increases (except in the Persistence Method, which stays consistent across the year). Since the Persistence Method accounts for no change in cloud whatsoever, it makes sense that its results are consistent across the entire year timeframe. Wind information changes on a daily bases in both speed and direction, so although the methodology of the other two methods is consistent, the wind is not, thus allowing for much more variability.

It is unfortunate that a daytime/nighttime satellite pass comparison could not be made independently of the length of time comparison. There is a slight difference in the 


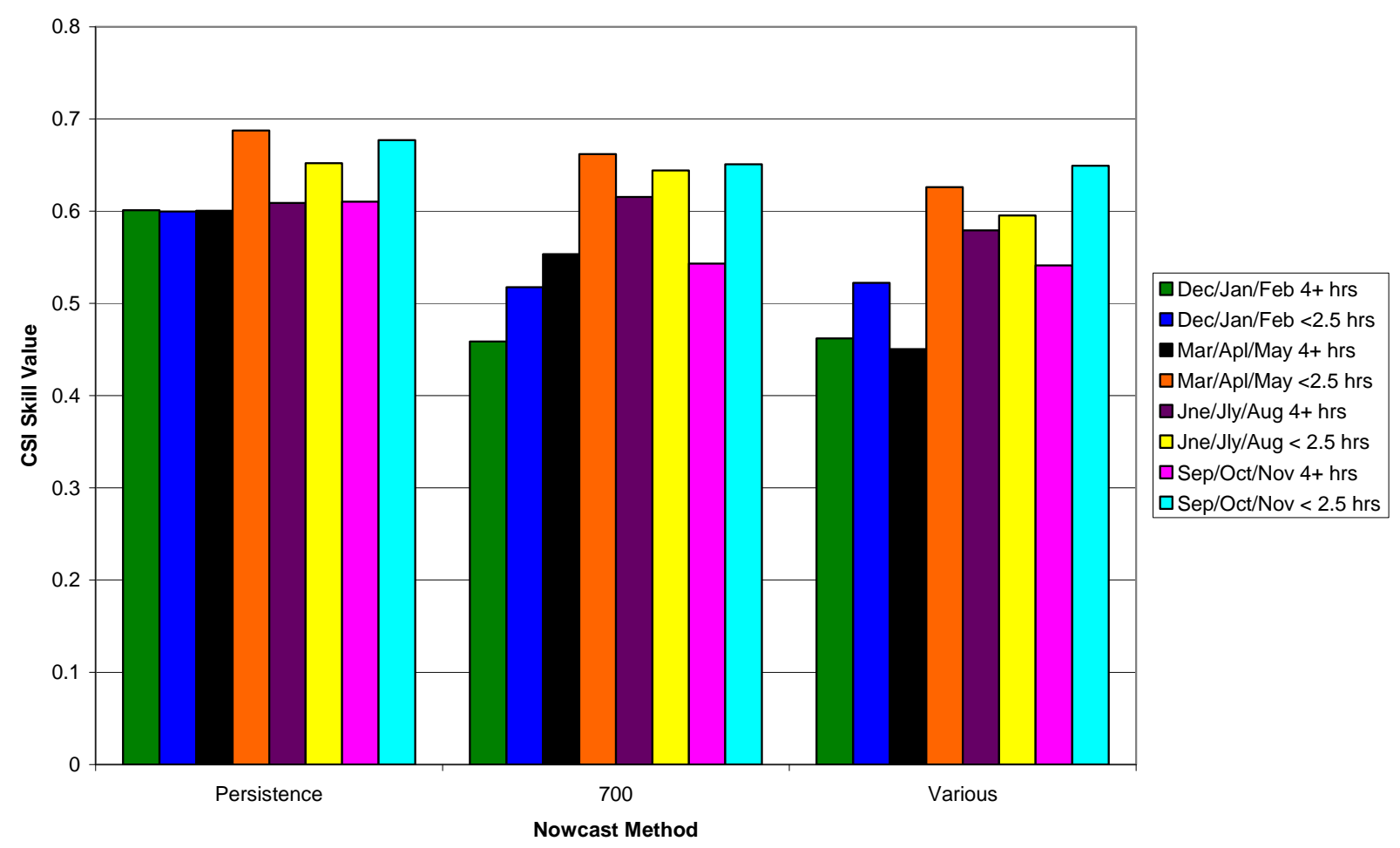

Fig 30: Comparison of season, method, and length of time CSI skill for each nowcast over Utah from December 2008 to November 2009.

way the MODIS Cloud Mask algorithm evaluates the presence of cloud during the day than at night (primarily the use of visible wavelength channels that only work during daytime hours). However, since every nowcast less than 2.5 hours occurs during the day and every 4-5 hour nowcast occurs during the nighttime, it is not possible to differentiate in nowcast differences due only to time of day. 


\subsection{Skill Domain Distribution}

Combining the various hits, misses, false alarms, and correct negatives over the region allows for plots of skill over an area to be plotted. Although our "total" values (Equations $13-16$ ) are beneficial when evaluating nowcasting skill over an area, it does not make sense to use these same scores when evaluating skill at one point. This is because, at any given point, unless there has been a similar number of both hits and correct negatives (thus letting the total CSI reach its maximum), skill plots will be misleading. Penalizing skill because a certain point just happens to be cloudy most of the time does not properly represent the distribution of skill at every point in a region. Thus, we choose to display plots of skill using the "cloudy" skill scores (Equations 5-8) and “clear" skill scores (Equations 9-12).

Figures (31) displays the percentage of time cloud is present over the region for each three month period. During the winter months, the northwest corner of Utah possesses the most clouds, especially over the Dugway region. However, during the springtime there are many more clouds in eastern Utah along the Uinta Mountain Range and in northern Utah along the Bear River Range. By summer, clouds become less frequent and do not seem to follow any type of pattern; but by autumn, as the jet stream begins to move south, clouds again begin to congregate along the Uinta and Wasatch Mountain Ranges.

Figure (32) displays "cloudy" CSI scores over Utah and southwest Wyoming for our major three forecast methods, while Figure (33) displays the corresponding "clear" CSI scores. There are many more bright colors for the two Persistence Method plots, indicating that that method does perform better than the other two. Interesting to note is 

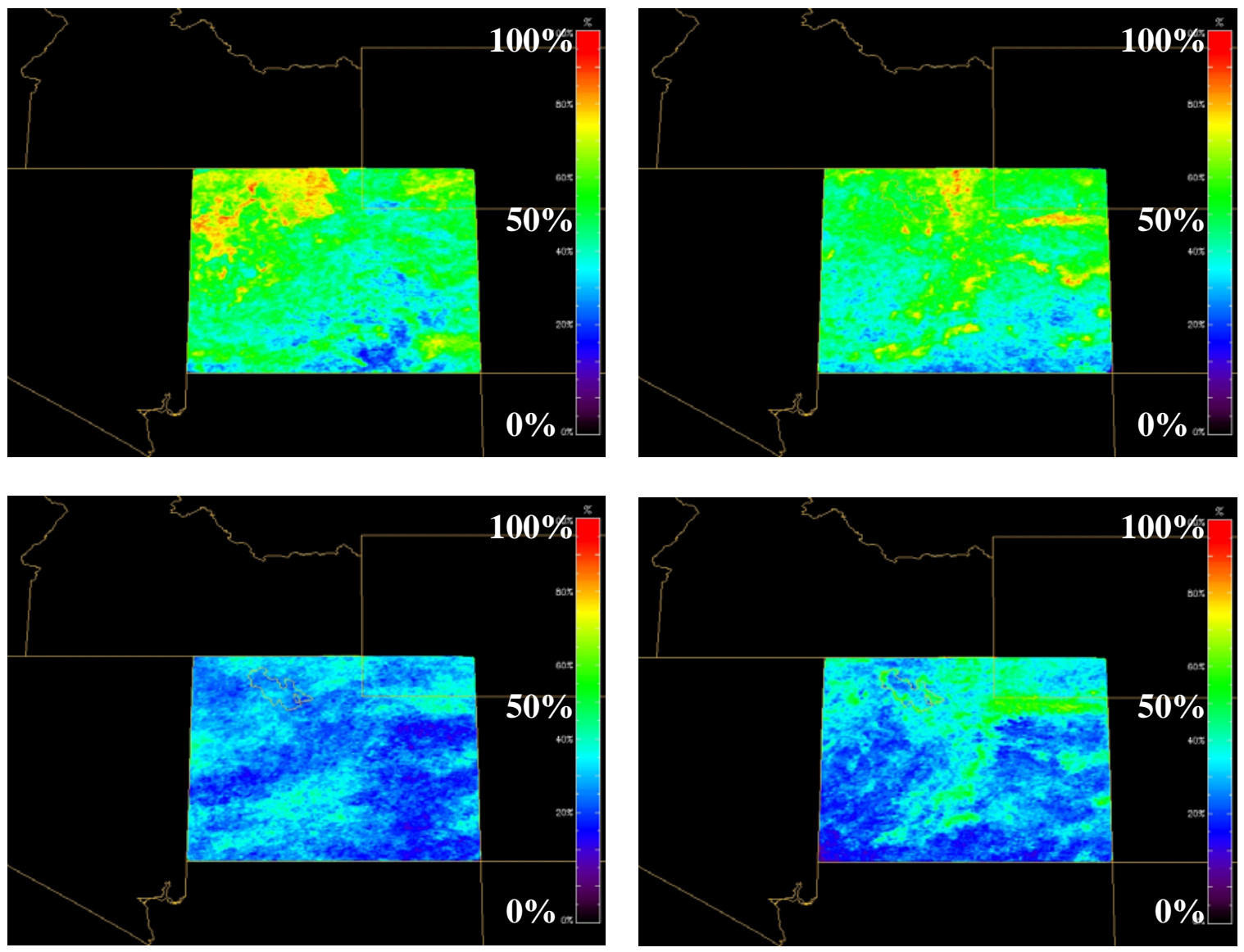

Fig 31: Cloud percentage over Utah and southwest Wyoming for December 2008 to February 2009 (top left), March to May 2009 (top right), June to August 2009 (bottom left), and September to November 2009 (bottom right).

the region of poor cloudy skill for the $700 \mathrm{mb}$ Wind method on the southeast corner of Utah, in addition to the region of poor clear skill for the Various Wind Method in Utah's northwest corner. 

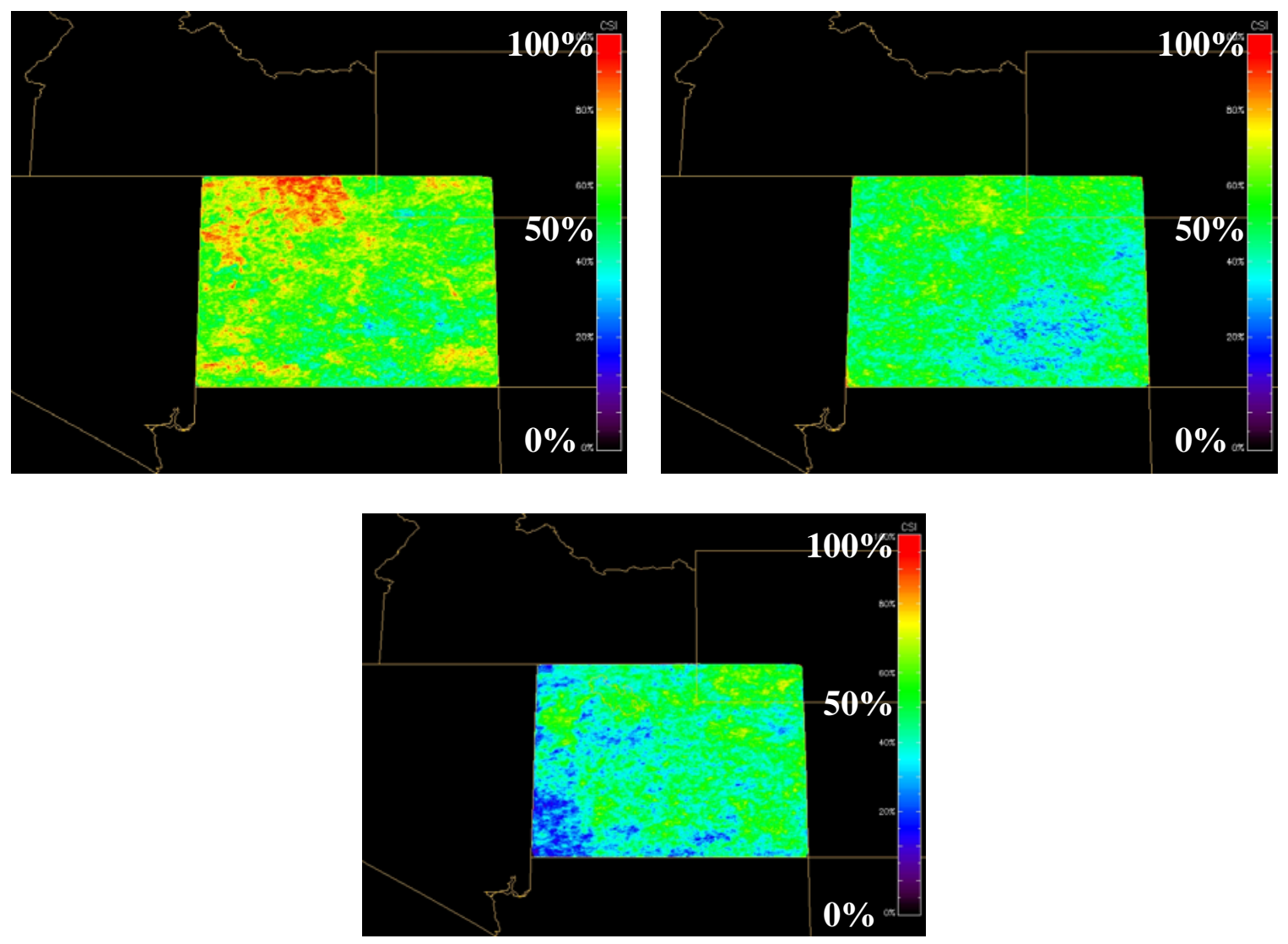

Fig 32: Cloudy CSI skill scores over Utah for the Persistence Method (top left), the 700mb Wind Method (top right) and the Various Wind Method (bottom) from December 2008 to February 2009. 

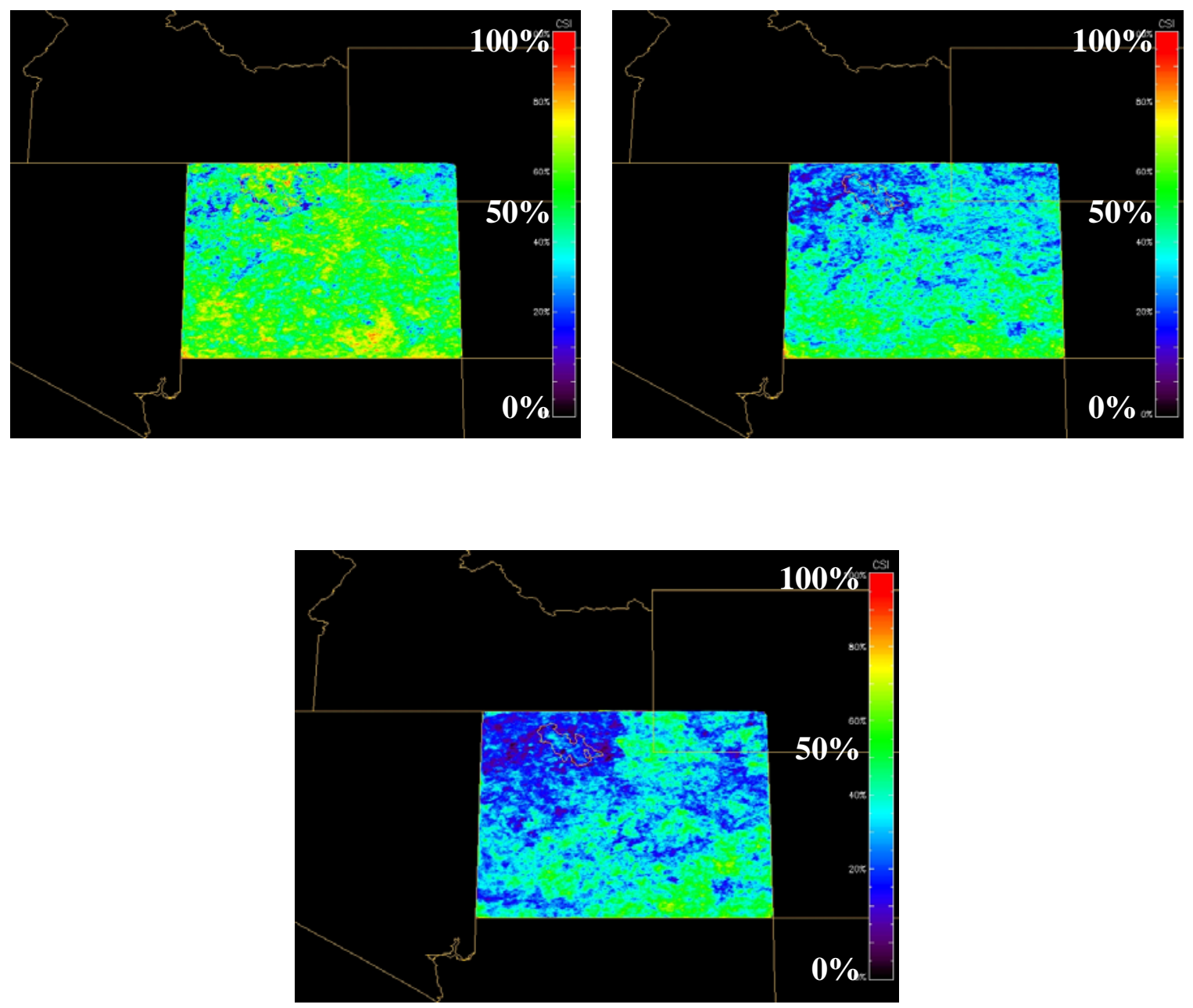

Fig 33: Clear CSI skill scores over Utah for the Persistence Method (top left), the 700mb Wind Method (top right) and the Various Wind Method (bottom) from December 2008 to February 2009.

Figure (34) and (35) displays cloudy and clear CSI skill scores over Utah from March to May 2009. In both cases, the Persistence and 700mb Methods did quite well, while the Various Wind Method did poorly. The Persistence Method was able to pick up on clouds in northern Utah along the Bear River Mountain Range better than any other method, while the Various Wind Method performed very poorly picking out clear areas near complex terrain. 

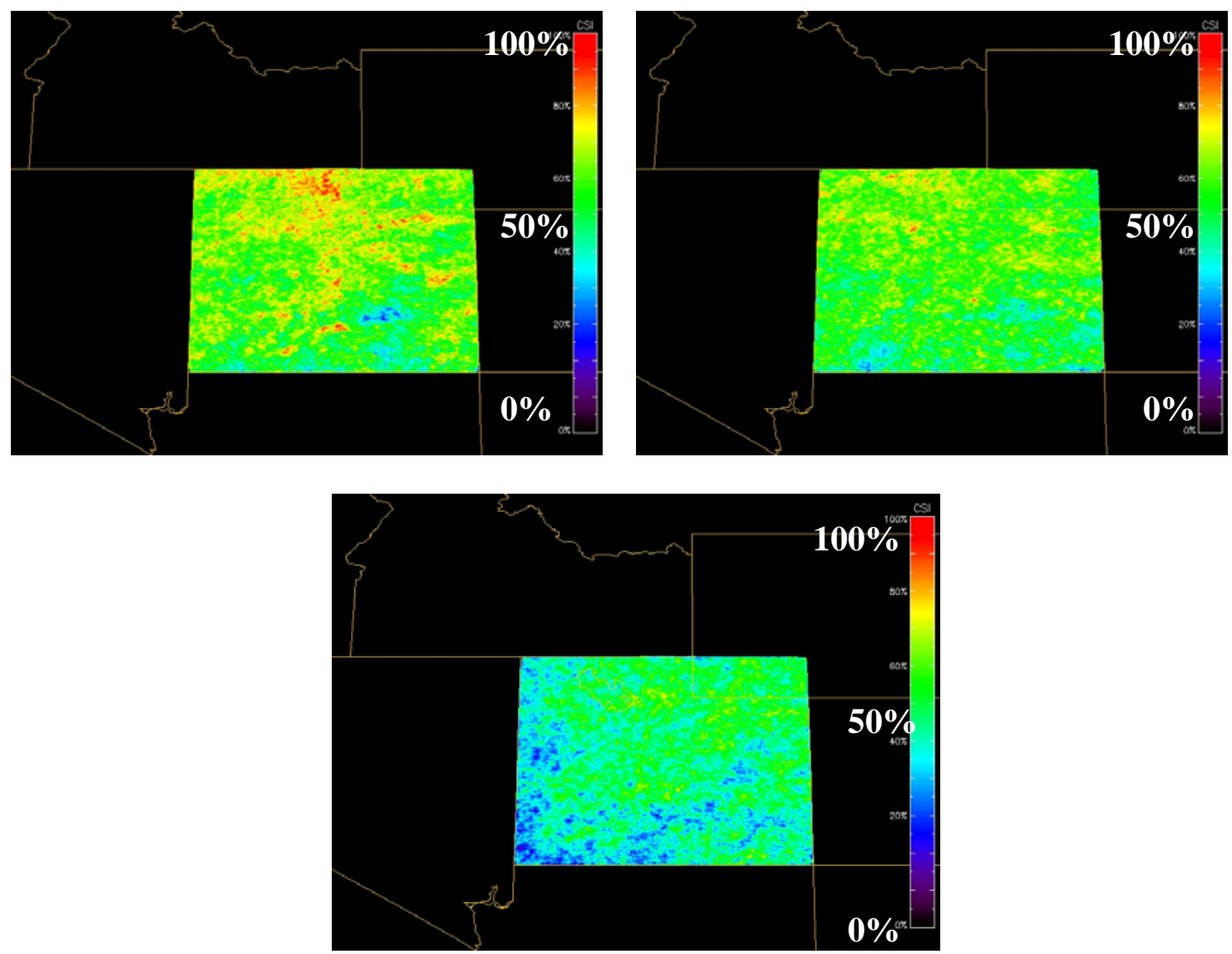

Fig 34: Cloudy CSI skill scores over Utah for the Persistence Method (top left), the 700mb Wind Method (top right) and the Various Wind Method (bottom) from March to May 2009. 

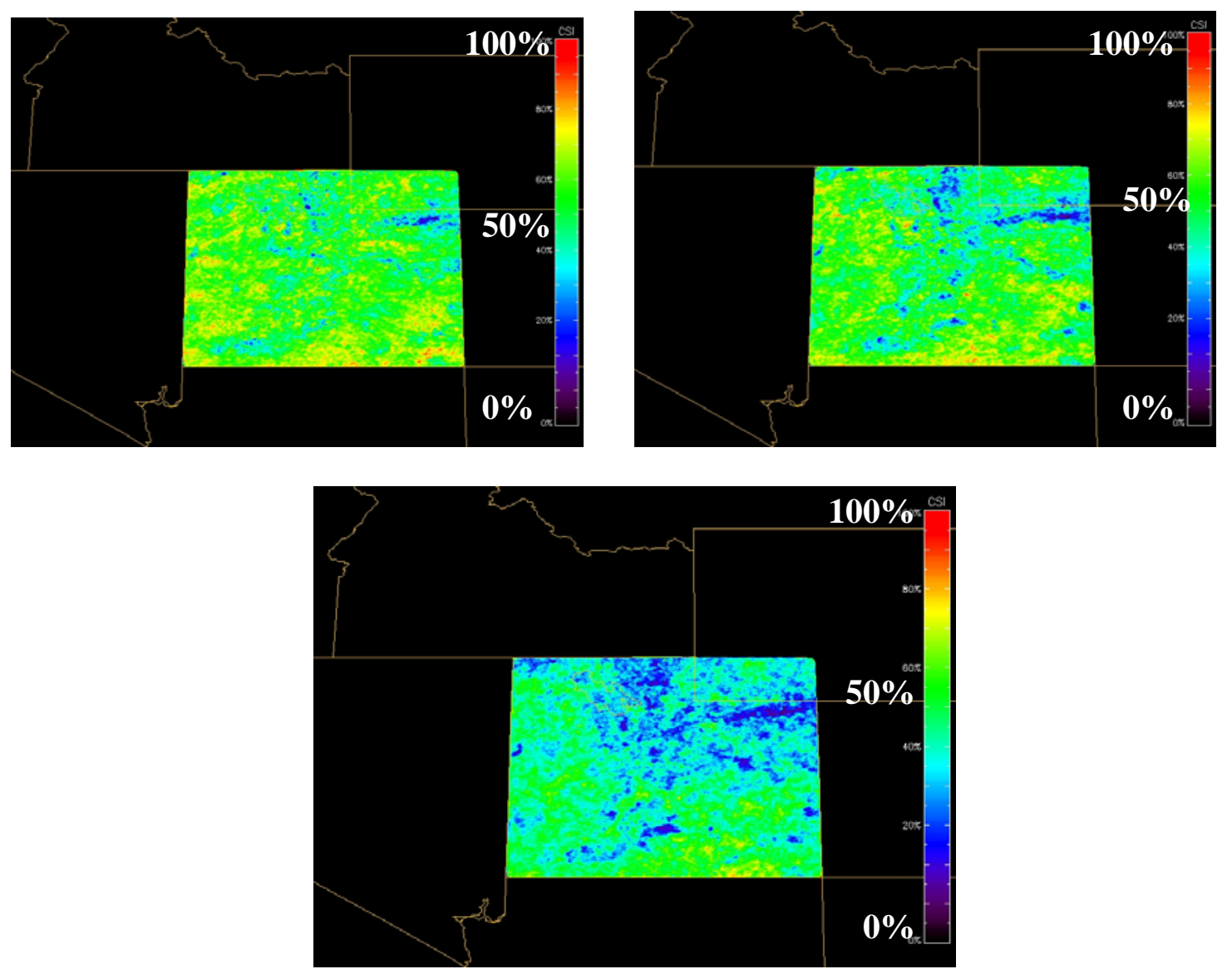

Fig 35: Clear CSI skill scores over Utah for the Persistence Method (top left), the 700mb Wind Method (top right) and the Various Wind Method (bottom) from March to May 2009.

Figures (36) and (37) show cloudy and clear CSI scores over Utah from June to August 2009. Immediately apparent are the warmer colors over the domain for the clear CSI scores, implying that all three methods do well at finding clear areas during these months. There appears to be no terrain-influenced cloudy or clear signal during the summer months, though this is to be expected since there are much fewer clouds in this region during this time. 

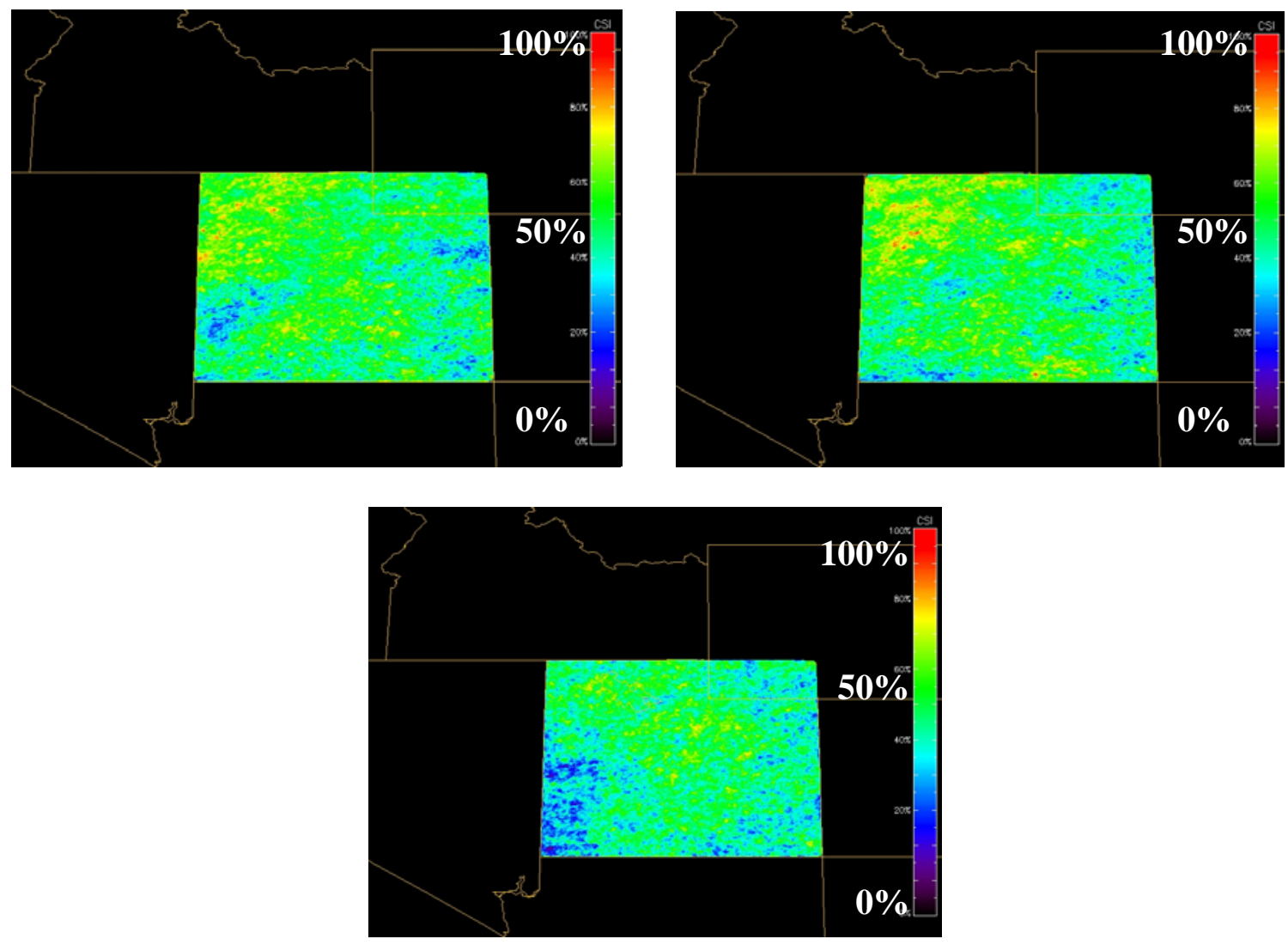

Fig 36: Cloudy CSI skill scores over Utah for the Persistence Method (top left), the 700mb Wind Method (top right) and the Various Wind Method (bottom) from June to August 2009. 

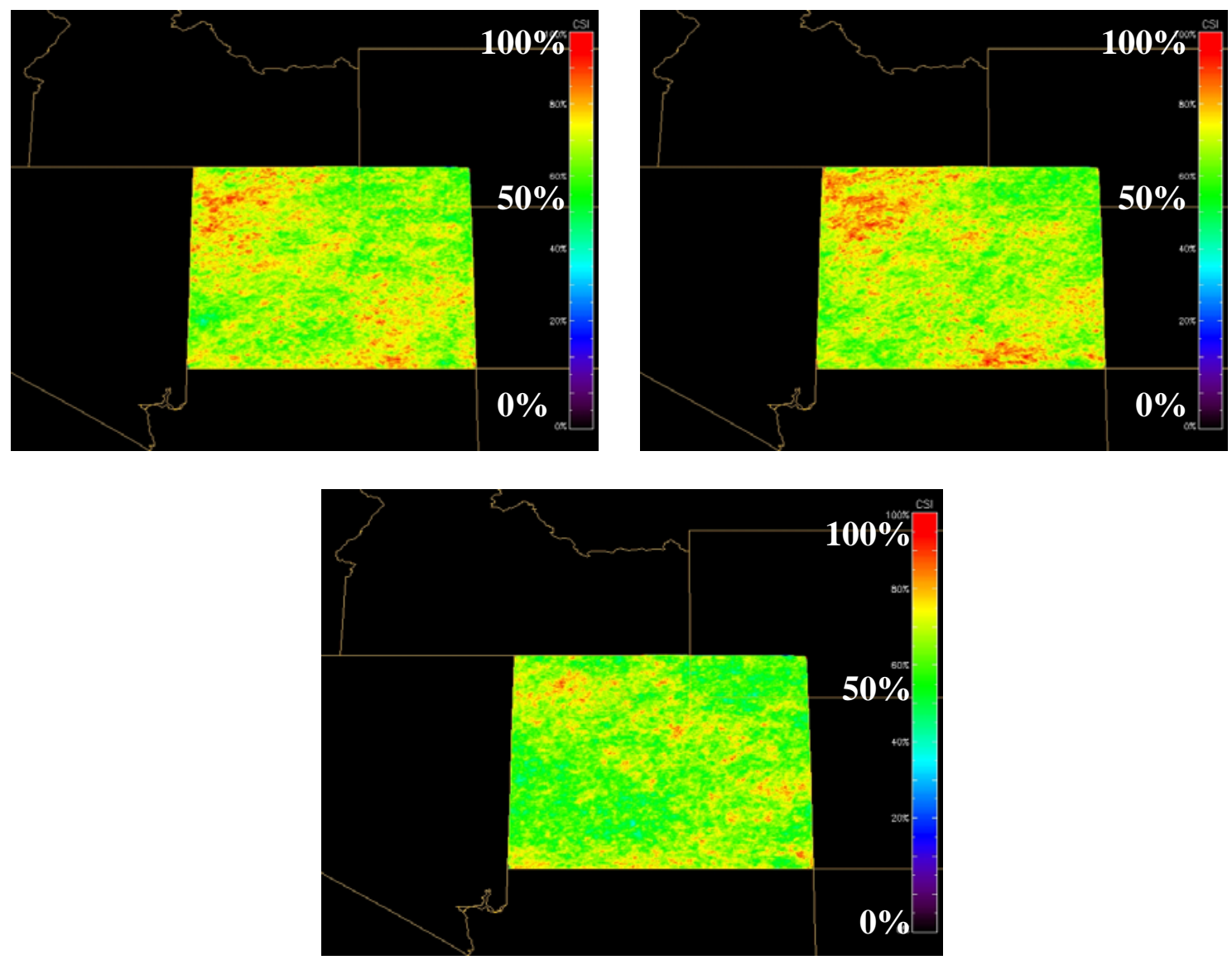

Fig 37: Clear CSI skill scores over Utah for the Persistence Method (top left), the 700mb Wind Method (top right) and the Various Wind Method (bottom) from June to August 2009.

Figure (38) and (39) show cloudy and clear CSI scores over Utah from September to November 2009. The Persistence Method is picking up on clouds over the Uinta Mountains, while the $700 \mathrm{mb}$ Wind and Various Wind methods are actually doing quite poor picking up on clear areas over the very same mountain range. In fact, the two displacement methods are poorly picking out any clear signal over most of the mountain ranges over Utah. This is likely due to clouds persisting over these mountains and not displacing with the wind. 

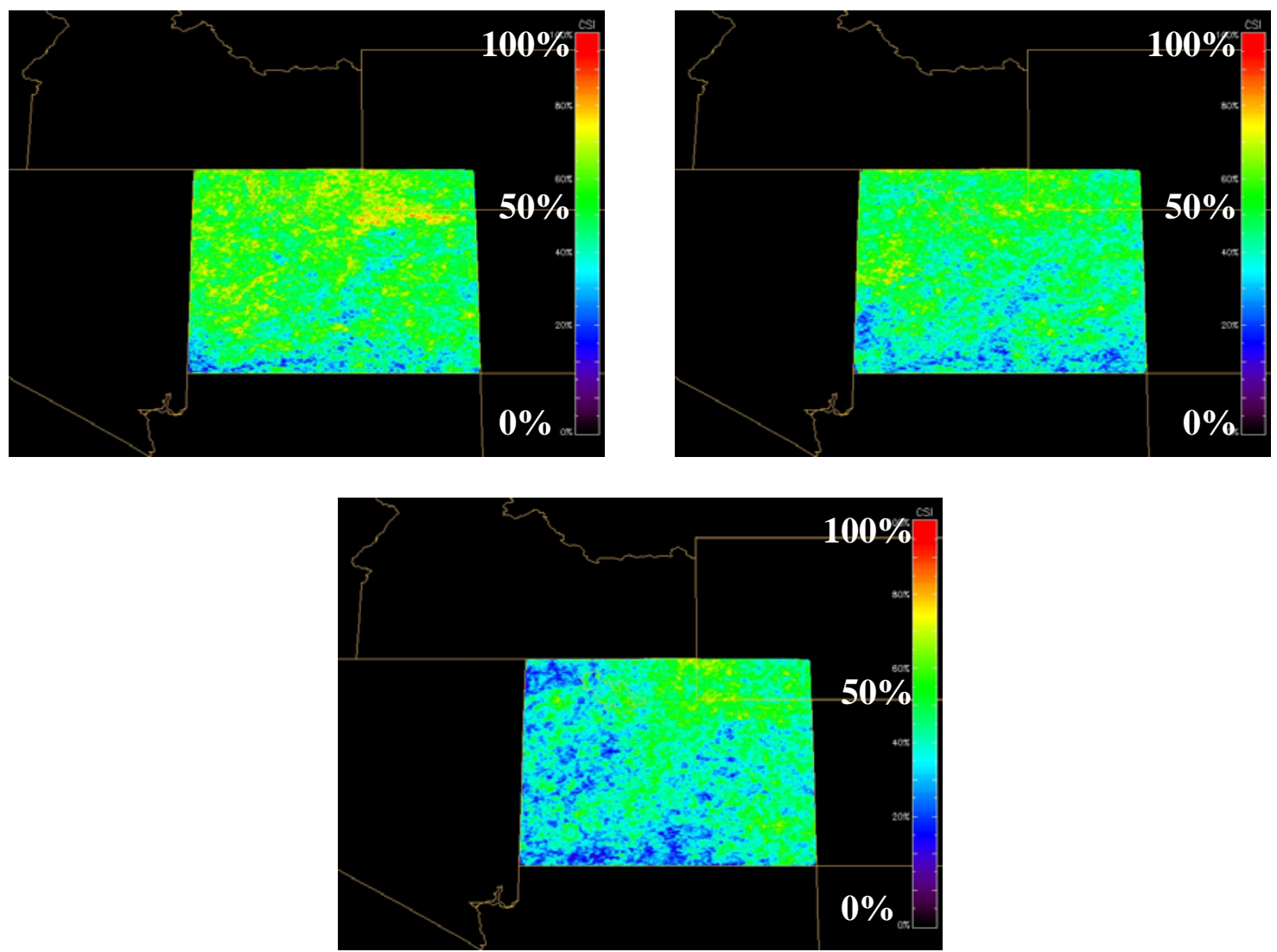

Fig 38: Cloudy CSI skill scores over Utah for the Persistence Method (top left), the 700mb Wind Method (top right) and the Various Wind Method (bottom) from September to November 2009. 

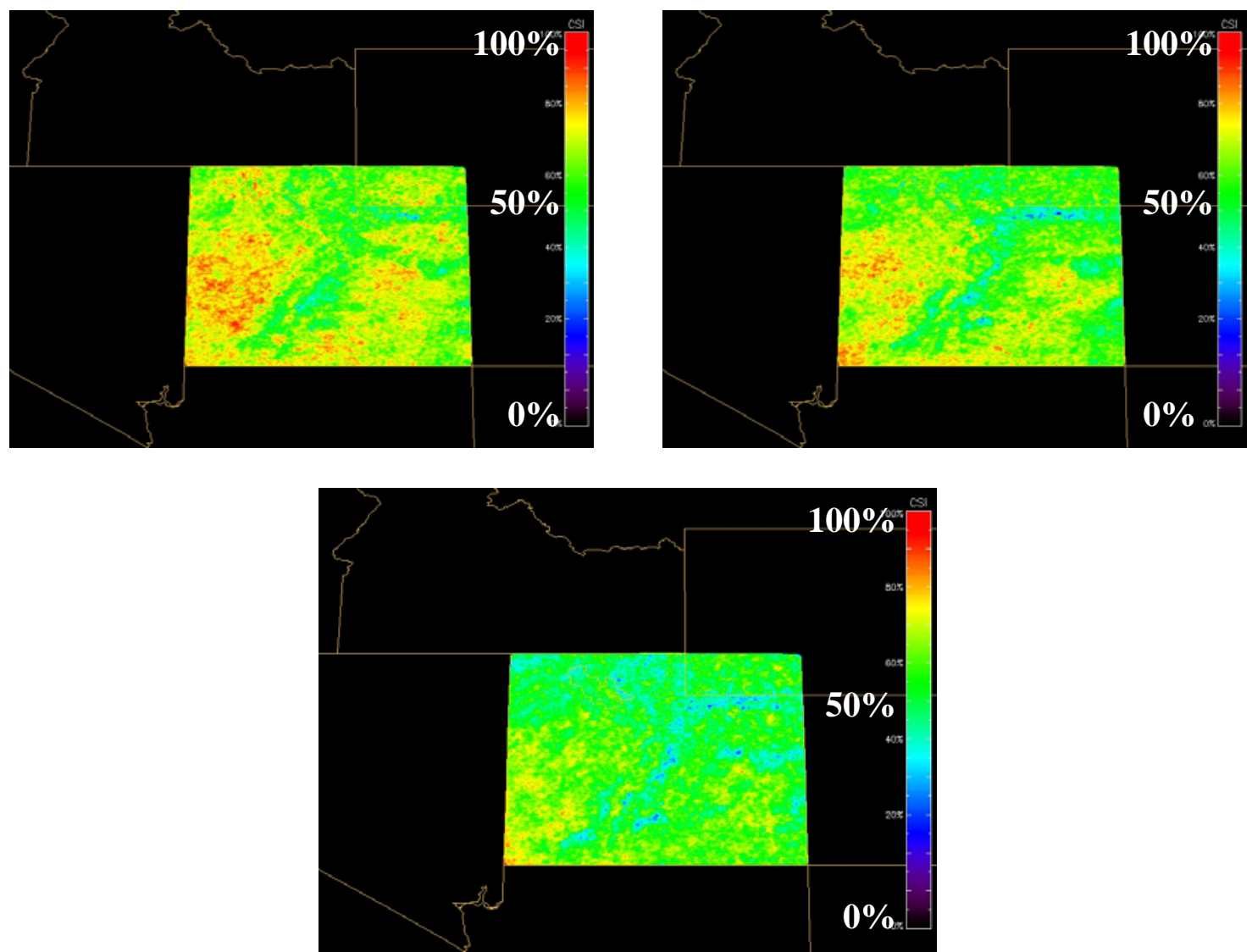

Fig 39: Clear CSI skill scores over Utah for the Persistence Method (top left), the 700mb Wind Method (top right) and the Various Wind Method (bottom) from September to November 2009. 


\subsection{Cloud Phase Skill Score Variations}

Using the MODIS Cloud Phase Algorithm, one can displace or persist certain cloud types depending on their microphysical makeup. Table (4) describes the various phases of clouds and divides them into one of six categories depending on which are persisted and which are displaced. These six categories are labeled as such in the skill score graphs that follow.

Table 4: Cloud phase category and cloud nowcasting behavior for each.

\begin{tabular}{|c|l|}
\hline Phase Category & \multicolumn{1}{c|}{ Cloud Phase Behavior } \\
\hline 1 & persist liquid water clouds, displace ice and mixed-phase clouds \\
\hline 2 & persist liquid water and mixed-phase clouds, displace ice clouds \\
\hline 3 & persist ice clouds, displace liquid water and mixed-phase clouds \\
\hline 4 & persist ice and mixed-phase clouds, displace liquid water clouds \\
\hline 5 & persist ice and liquid water clouds, displace mixed-phase clouds \\
\hline 6 & persist mixed-phase clouds, displace ice and liquid water clouds \\
\hline
\end{tabular}

Figure (40) shows various skill scores for the different cloud phase

persistence/displacement schemes. During the winter months over Utah, the $4^{\text {th }}$ method (persist ice and mixed-phase clouds while displacing liquid water clouds) performs the best, beating the $1^{\text {st }}$ method (persisting liquid water clouds while displacing ice and mixed-phase clouds) by $\sim 9 \%$ CSI. This may be due to very deep cloud decks associated with mid-latitude cyclones that do not move very quickly during the winter months. Persisting ice and liquid water clouds by displacing mixed-phase clouds (method 5) also performed almost as well as method 4 . 


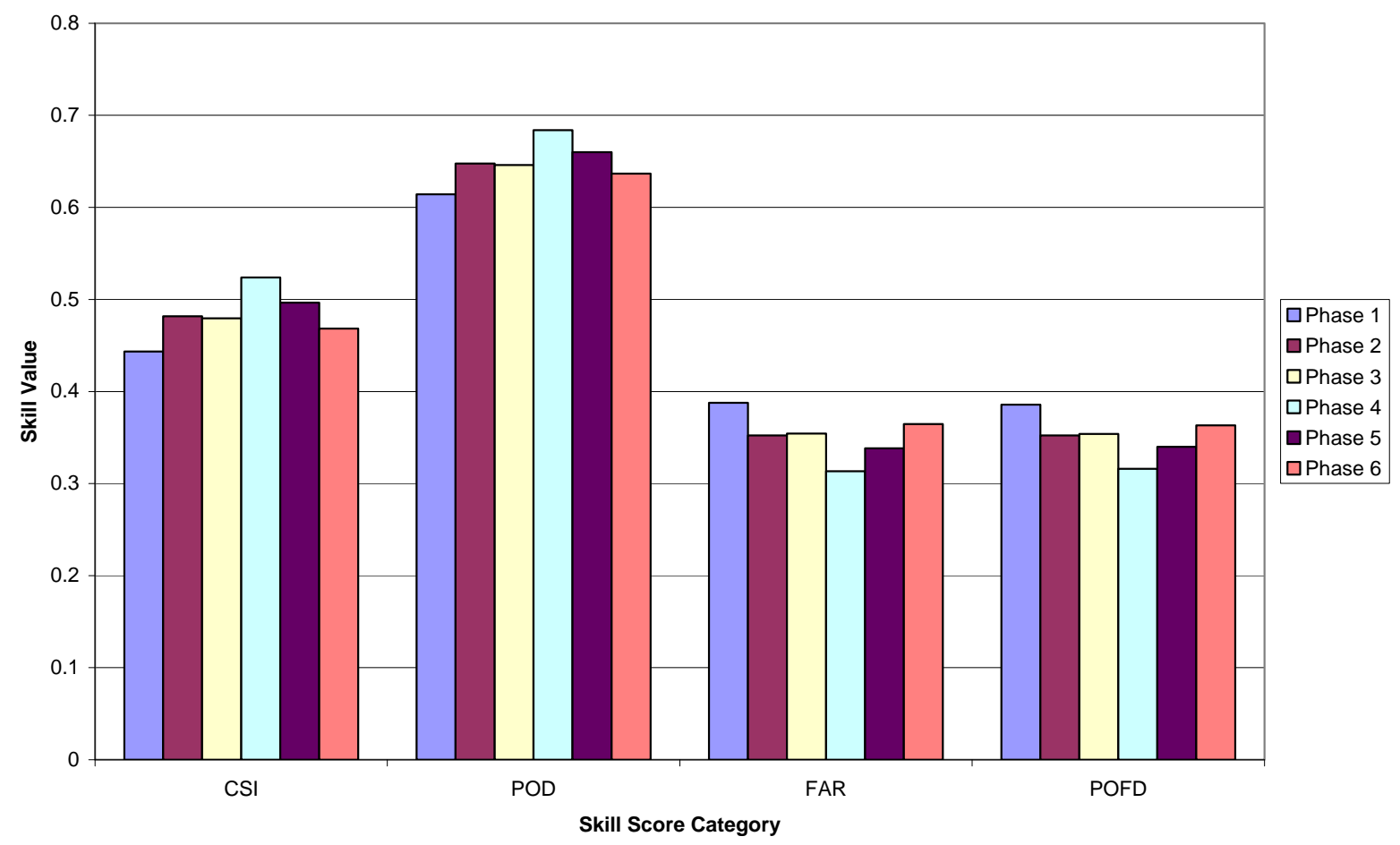

Fig 40: Cloud phase skill scores from December 2008 to February 2009 over Utah.

Figure (41) continues the skill scores from (40) with TSS and PFC values. Again, the $4^{\text {th }}$ method performs the best, while persisting liquid water clouds while displacing mixed-phase and ice clouds (method 1) performs the worst. This is most likely due to clouds being labeled as ice actually being quite deep, thus they move at one slow speed rather than get sheared off into pieces, especially in the winter months. 
Cloud Phase TSS and PFC over Utah from 1 Dec 2008 to 28 Feb 2009

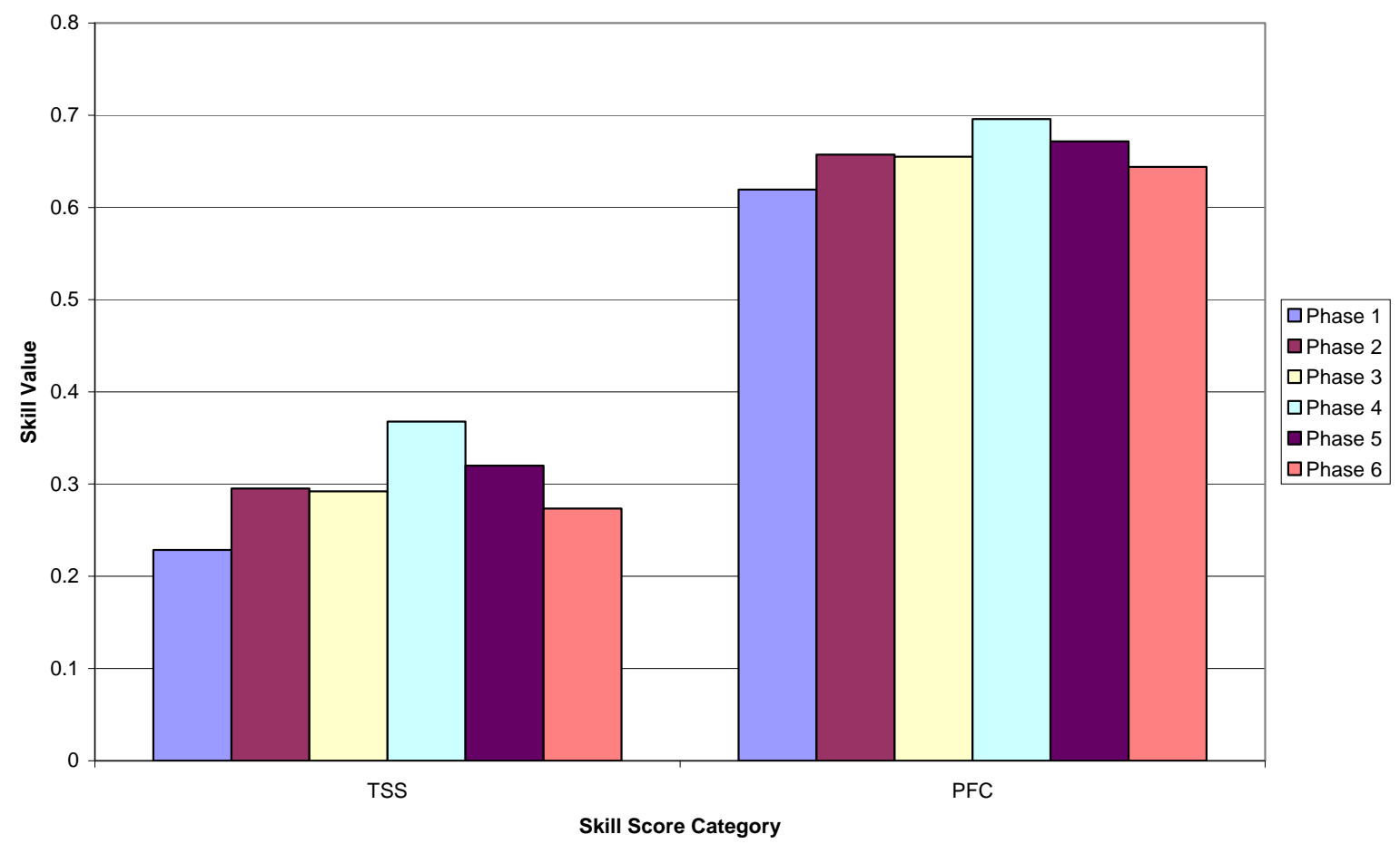

Fig 41: True Skill Statistic and Percentage of Forecasts Correct values for six cloudphase displacement/persistence methods over Utah from December 2008 to February 2009. 


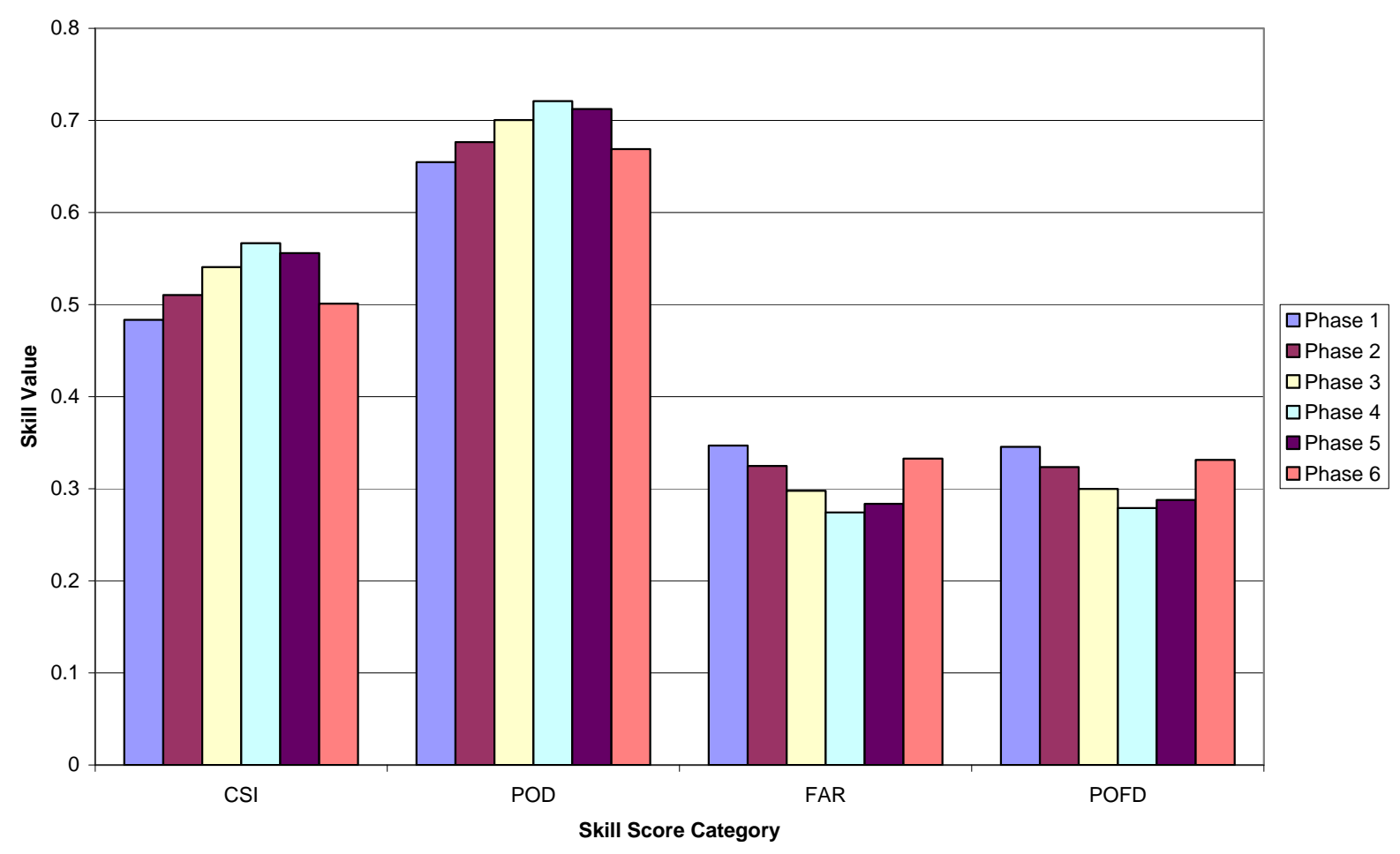

Fig 42: Cloud phase skill scores from March to May 2009 over Utah.

Figure (42) shows the cloud phase skill scores from March to May 2009 over Utah, and Figure (43) shows the TSS and PFC for the same region during the same timeframe. The skill scores all improved over the winter months, but that is because displacement methods perform better during spring than during winter over our domain (see Section 5.3). Method 4 is sill the best for displacing/persisting cloud based on phase, with Method 1 still performing most poorly. Method 5 is still a close second; however since both methods 5 and 6 persist ice clouds, it is most likely that that effect is the dominating factor. 


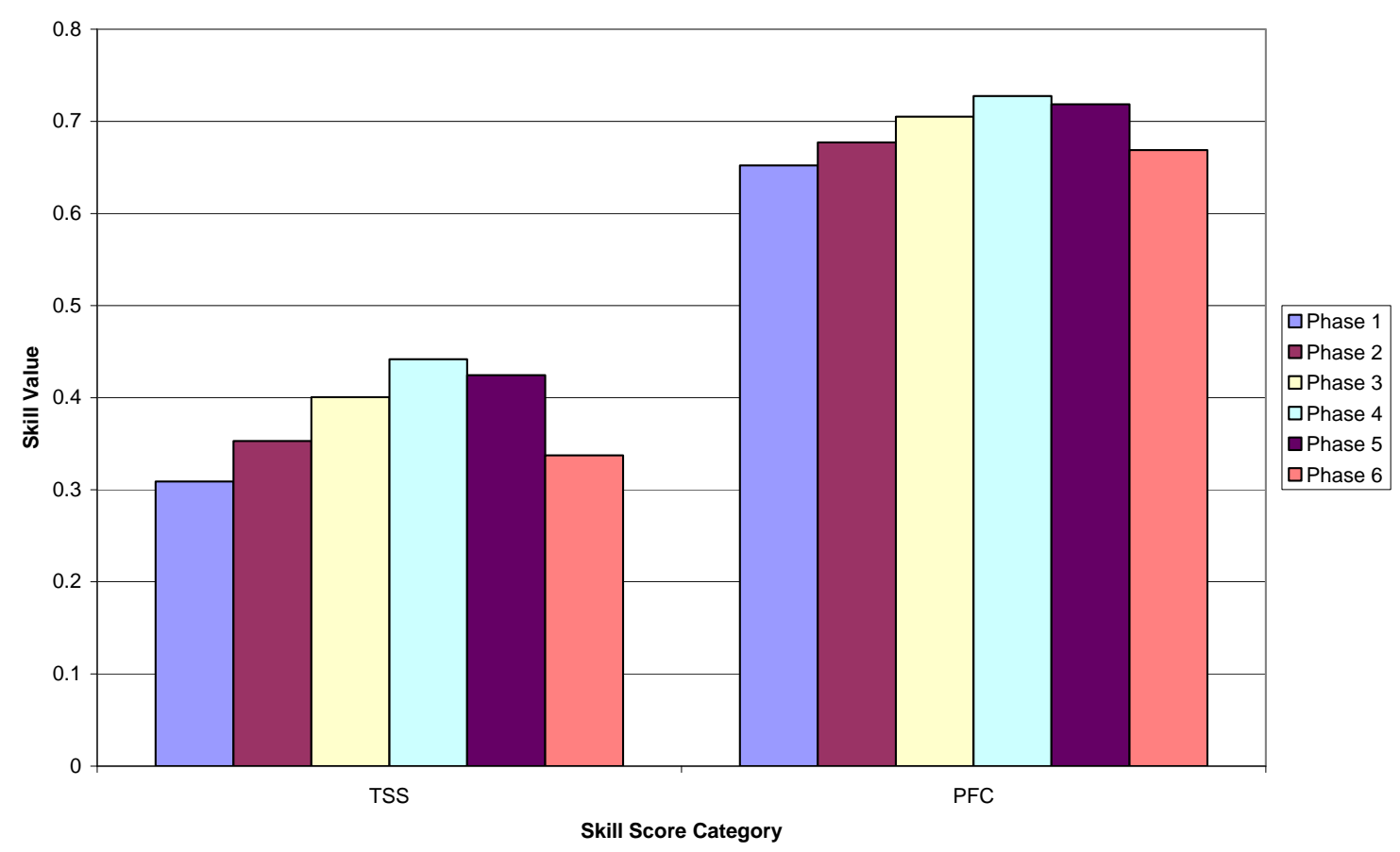

Fig 43: TSS and PFC values for six cloud-phase displacement/persistence methods over Utah from March to May 2009.

Figures (44) and (45) cloud phase show skill scores for the summer months over Utah in 2009. Methods 3, 4, and 5 all show similar skill, due in part to displacement methods performing much closer to persistence methods during this time of year. Summer days are also more likely to have lower, liquid water clouds than other months, which would explain why Method 3 (persist ice clouds, displace liquid water and mixedphase clouds) performs so similarly to Methods 4 and 5. 
Cloud Phase Skill Scores over Utah from 1 June 2009 to 31 August 2009

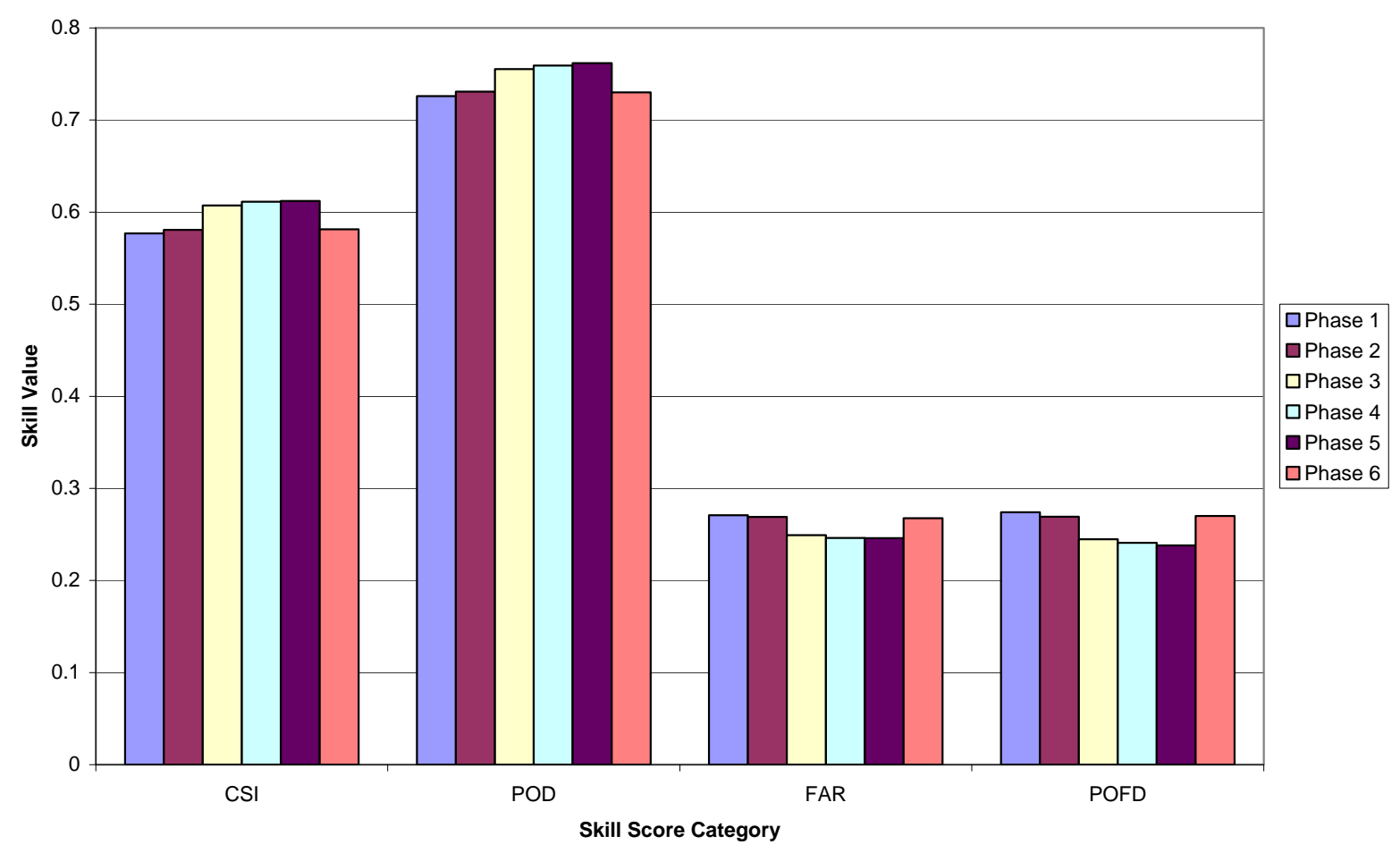

Fig 44: Cloud phase skill scores from June to August 2009 over Utah. 


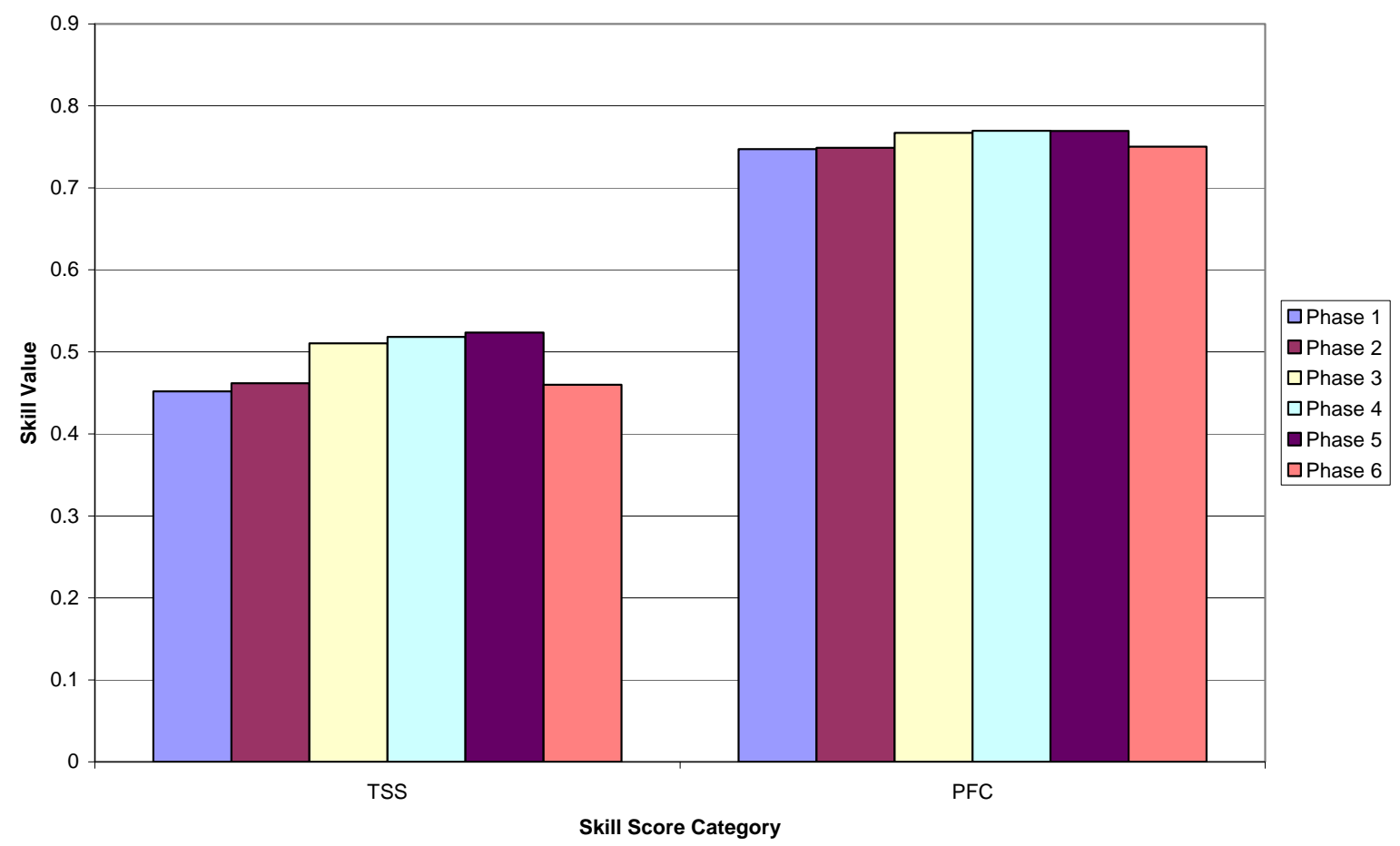

Fig 45: TSS and PFC values for six cloud-phase displacement/persistence methods over Utah from June to August 2009.

Finally, Figures (46) and (47) finish the year over Utah will cloud phase skill scores from September to November 2009. Again, Method 4 performs best with Method 5 in a close second. It should be noted that in all of these cases, the Persistence Method from Section 5.2 still performs better every month than any of these cloud phase methods. This is because as soon as any type of displacement scheme modifies persistence, the skill will decrease since pure persistence has proven to be the best nowcast for clouds in this region. 
Cloud Phase Skill Scores over Utah from 1 September 2009 to 30 November 2009

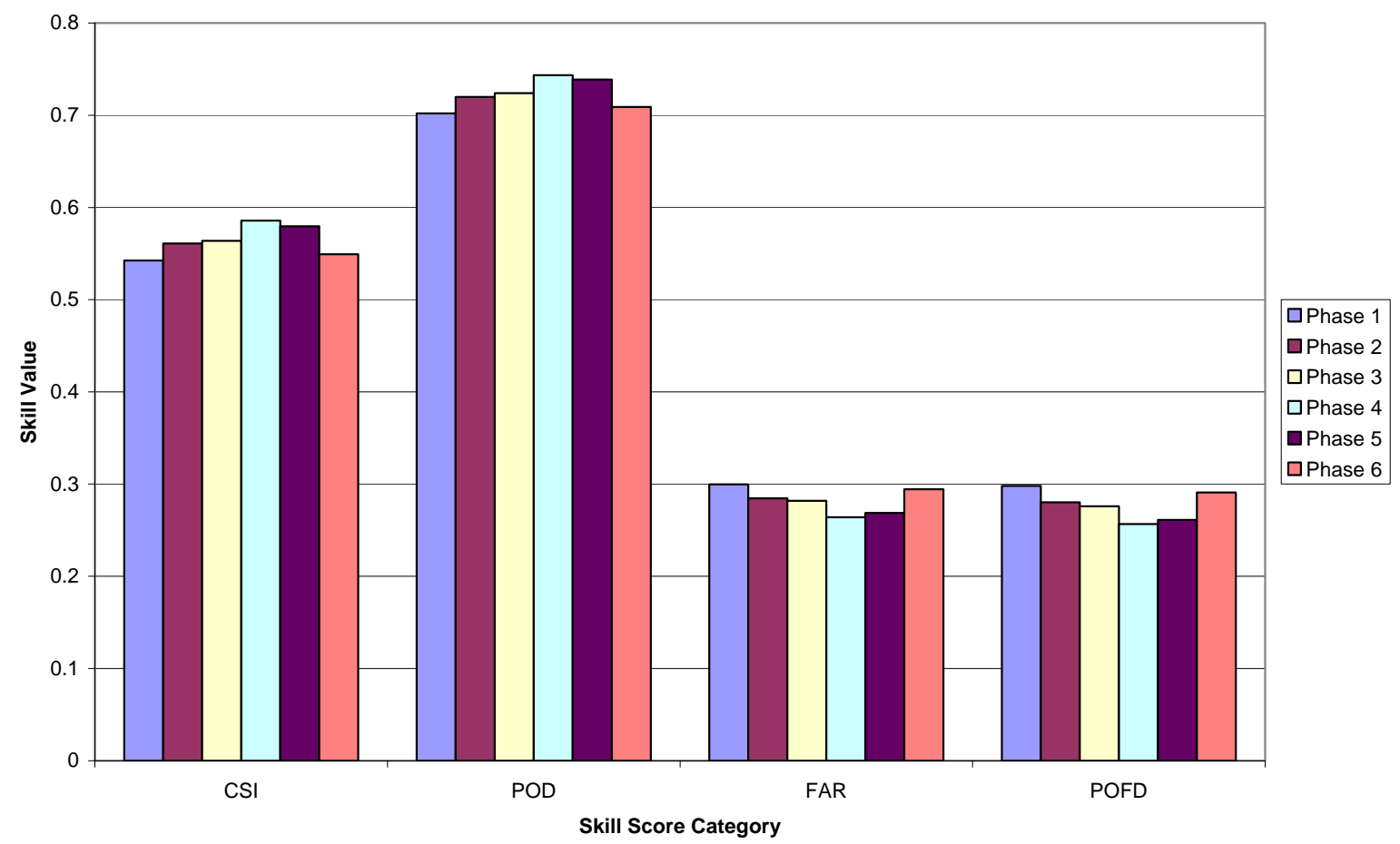

Figure 46: Cloud phase skill scores from September to November 2009 over Utah. 
Cloud Phase Skill Scores over Utah from 1 September 2009 to November 302009

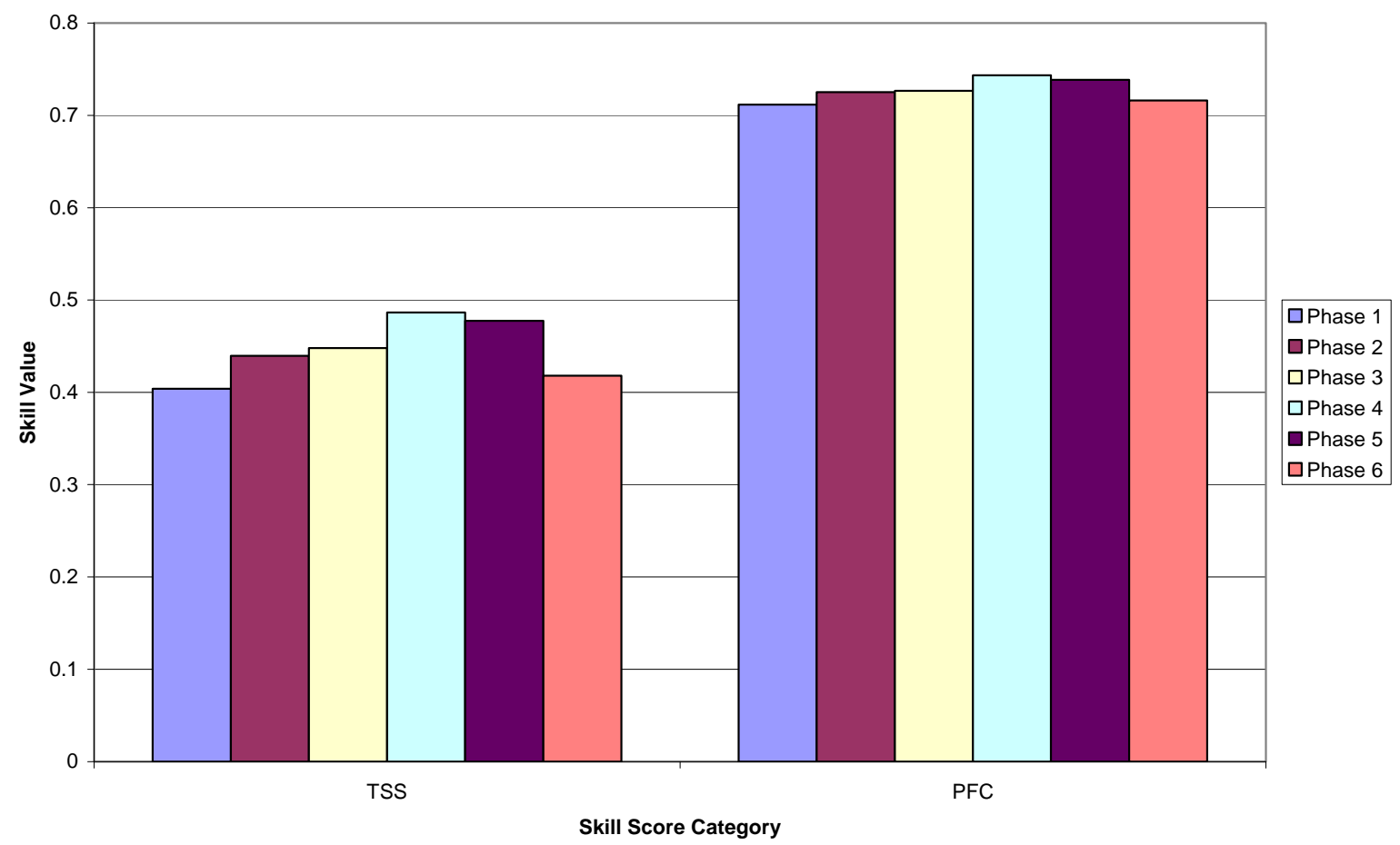

Figure 47: TSS and PFC values for six cloud-phase displacement/persistence methods over Utah from September to November 2009. 


\subsection{Synoptic Weather Contributions to Nowcasting Skill}

Figure (48) shows a time series of CSI skill scores over Utah for the month of December in 2008 for the Persistence Method, 700mb Wind Method, and the Various Winds Method. It is apparent that there are certain situations in which one nowcasting method performs much better than the other two, or all of them perform quite well, or all of them perform quite poorly. Because of the way CSI scores behave mathematically, we define skill scores as "poor" by being less than or equal to 50\% CSI, while CSI scores that perform "well" being greater than $50 \%$.

Six days each during the months of December, March, June, and September were chosen over Utah to analyze the synoptic weather situation present around the time of the

CSI for Dec 2008 over Utah (15 cases)

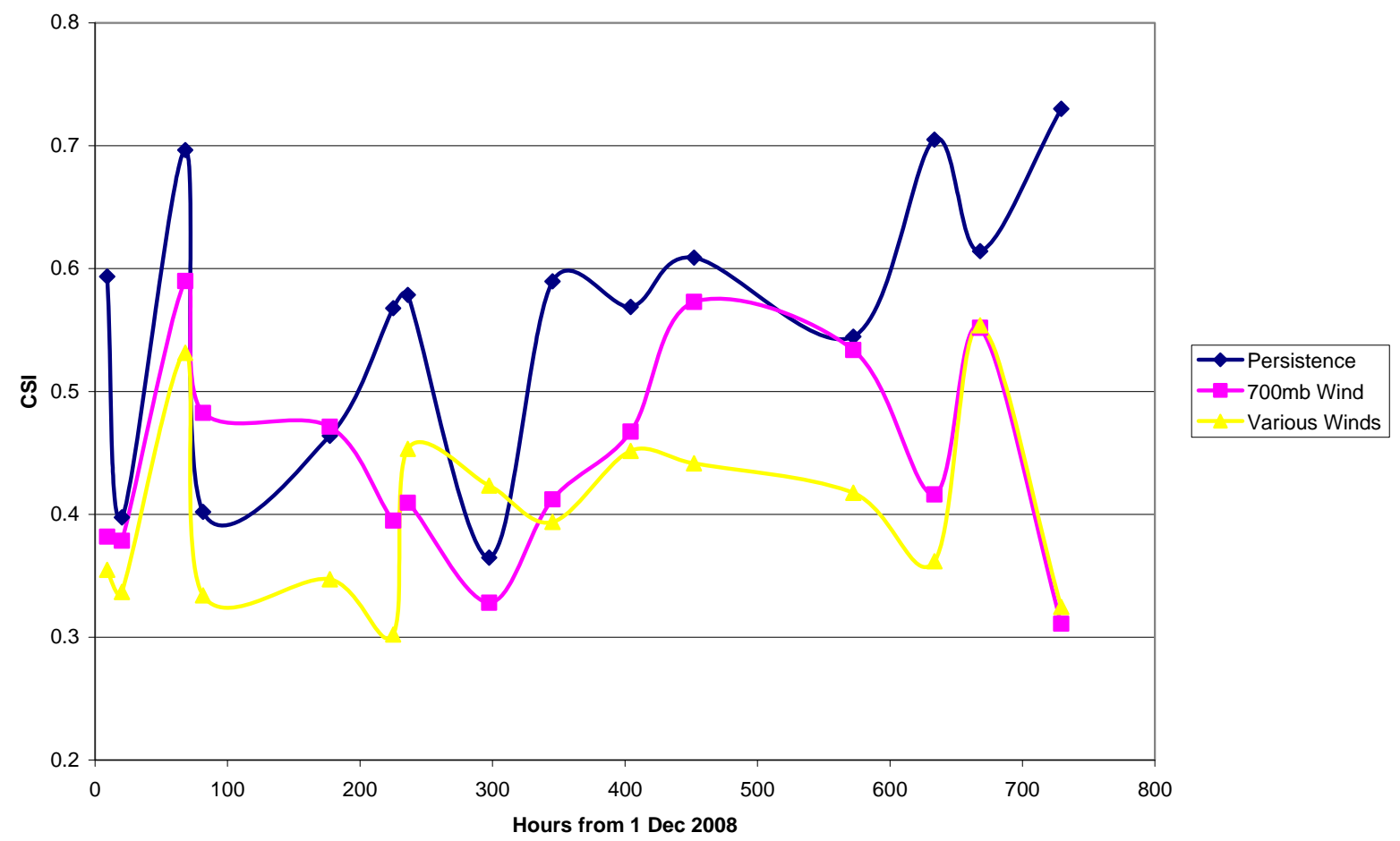

Fig 48: Time series of CSI Skill Scores for all 15 cases over Utah for December 2008. 
Table 5: Twenty-four specific cases and the reasons why they were chosen from the year of data over Utah from December 2008 to November 2009.

\begin{tabular}{|c|c|}
\hline \multicolumn{2}{|r|}{ Dec-08 } \\
\hline Julian Day & Why? \\
\hline 336 & Persistence does well, 700 and Various do poorly \\
\hline 338 & All three do well \\
\hline 348 & All three do poorly \\
\hline 362 & Persistence does well, 700 and Various do poorly \\
\hline 363 & All three do well \\
\hline 366 & Persistence does well, 700 and Various do poorly \\
\hline \multicolumn{2}{|r|}{ Mar-09 } \\
\hline Julian Day & Why? \\
\hline 61 & All three do well \\
\hline 62 & Persistence and 700 do well, Various does poorly \\
\hline 75 & Persistence does well, 700 and Various do poorly \\
\hline 75 & All three do well \\
\hline 80 & All three do poorly \\
\hline 85 & Persistence and 700 do well, Various does poorly \\
\hline \multicolumn{2}{|r|}{ Jun-09 } \\
\hline Julian Day & Why? \\
\hline 153 & All three do poorly \\
\hline 162 & Persistence and 700 do well, Various does poorly \\
\hline 165 & All three do poorly \\
\hline 172 & All three do poorly \\
\hline 174 & All three do well \\
\hline 181 & Persistence and 700 do well, Various does poorly \\
\hline \multicolumn{2}{|r|}{ Sep-09 } \\
\hline Julian Day & Why? \\
\hline 256 & All three do poorly \\
\hline 258 & Persistence and 700 do well, Various does poorly \\
\hline 258 & All three do poorly \\
\hline 260 & Persistence and Various do well, 700 does poorly \\
\hline 263 & All three do poorly \\
\hline 267 & All three do well \\
\hline
\end{tabular}

cloud nowcast. These days were chosen because either all three nowcasting methods obtained CSI skill scores greater than $50 \%$, all three had CSI scores less then $50 \%$, or one out of the three performed poorly while the other two performed well. Table (5) summarizes the breakdown of days and the reason why they were chosen. 


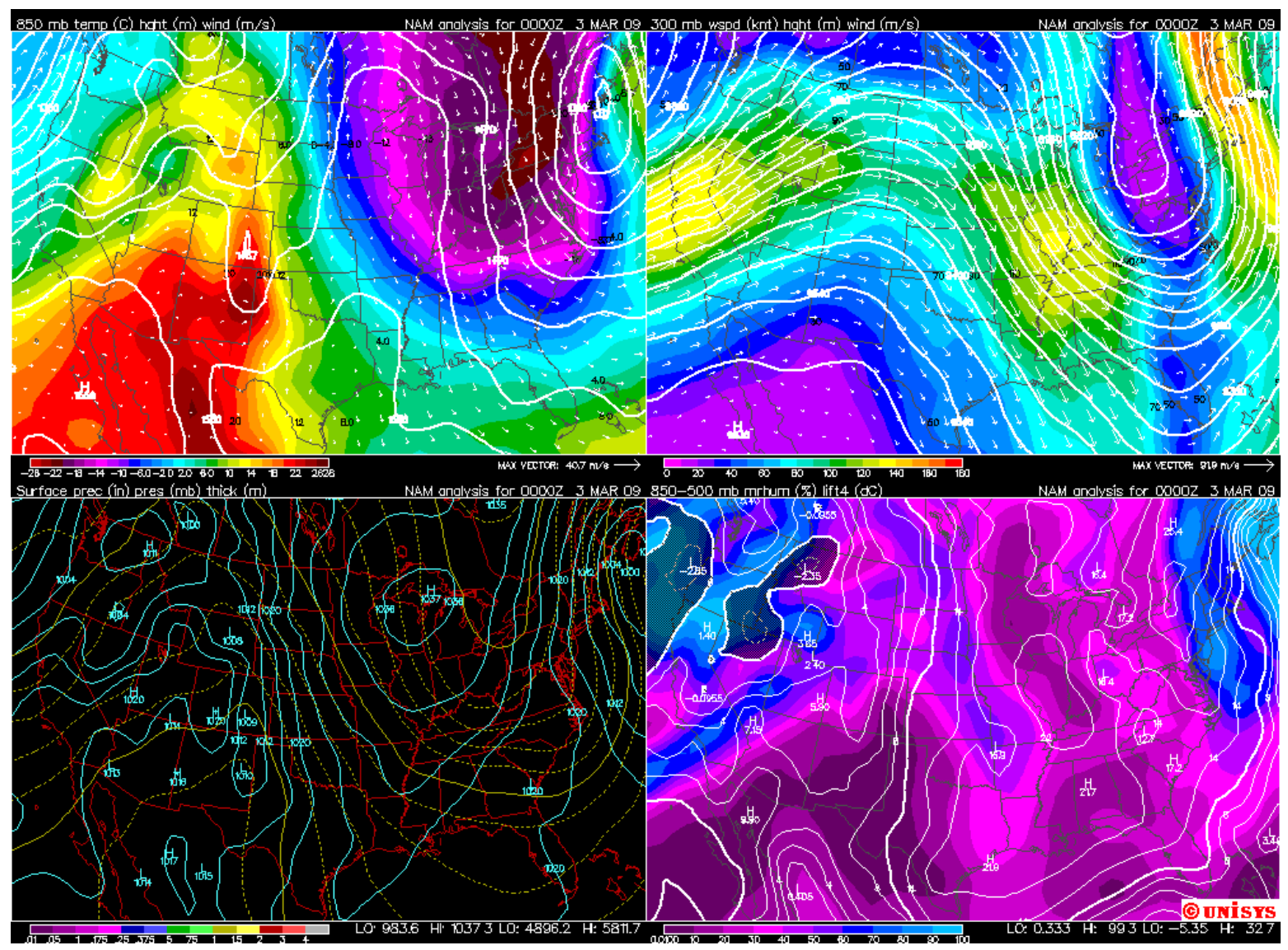

Fig 49: Example of synoptic conditions leading to a nowcasting situation in which all three methods perform well (Unisys 2010).

Data from the initialized NAM model runs at $00 \mathrm{z}$ and $12 \mathrm{z}$ were used to diagnose the synoptic situation for each chosen day in the hopes of finding a reason why some nowcasting techniques work better than others in certain situations.

Analysis of the various days showed that situations in which all three nowcasting methods performed well (six cases), winds aloft were relatively weak and an upper-level ridge was either in place or moving in to the region. This makes sense because a ridge will mitigate most vertical motion, thus keeping many new clouds from forming and 


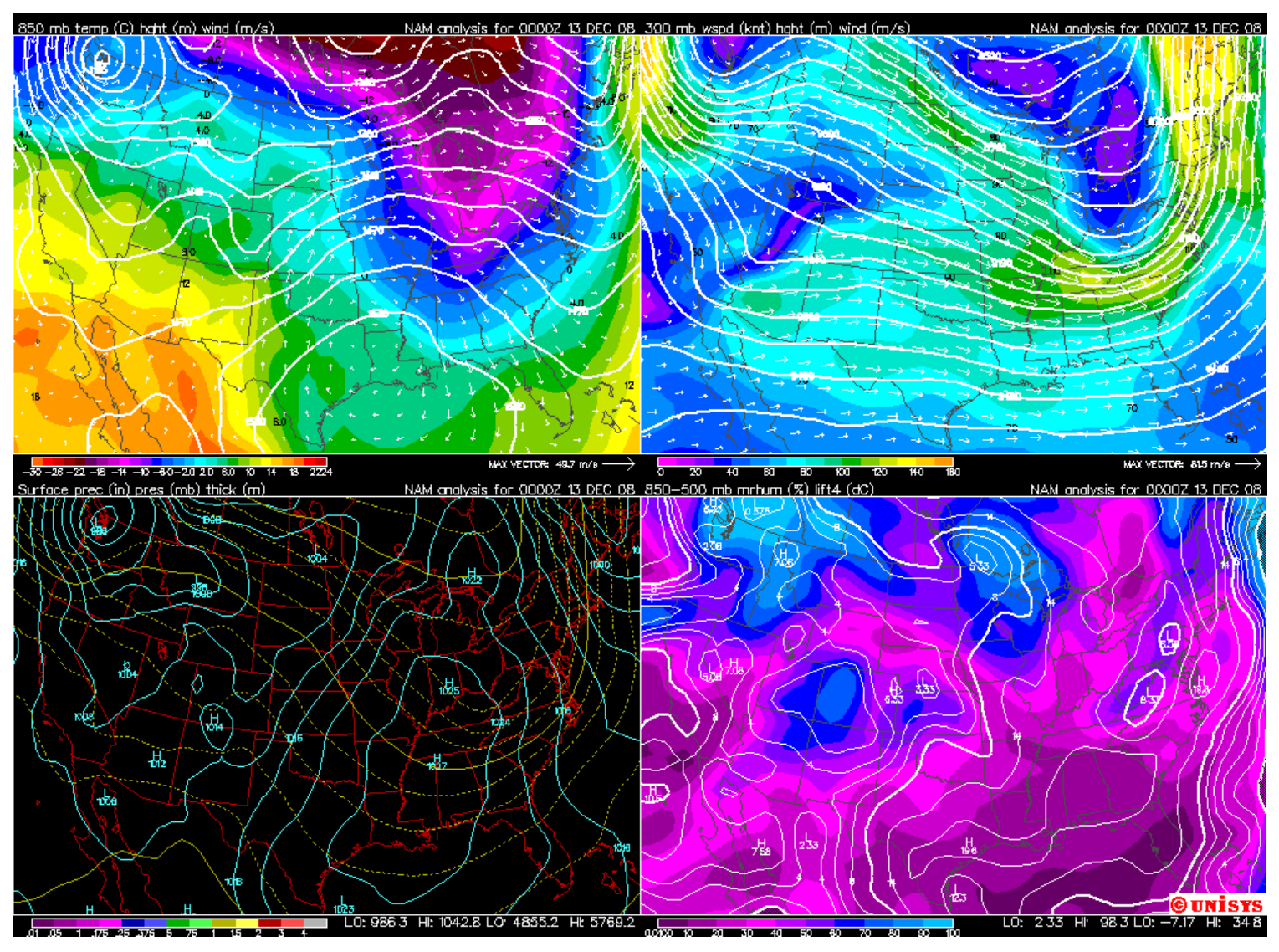

Fig 50: Example of synoptic conditions leading to a nowcasting situation in which all three methods perform poorly (Unisys 2010).

throwing off the various displacement nowcasting techniques by adding or subtracting clouds from the region. An example of such a day can be seen in Figure (49).

The days in which all three cloud nowcasting methods performed poorly (eight cases) show a strong pattern of changing weather conditions. In every situation, an upper-level shortwave, an upper-level trough, or a deep closed low-pressure system was either directly over Utah or moving into the region during the nowcasting period. This is not surprising since any type of upper-level forcing is going to cause clouds to generate and regenerate in seemingly random places. Because these nowcasting methods assume 


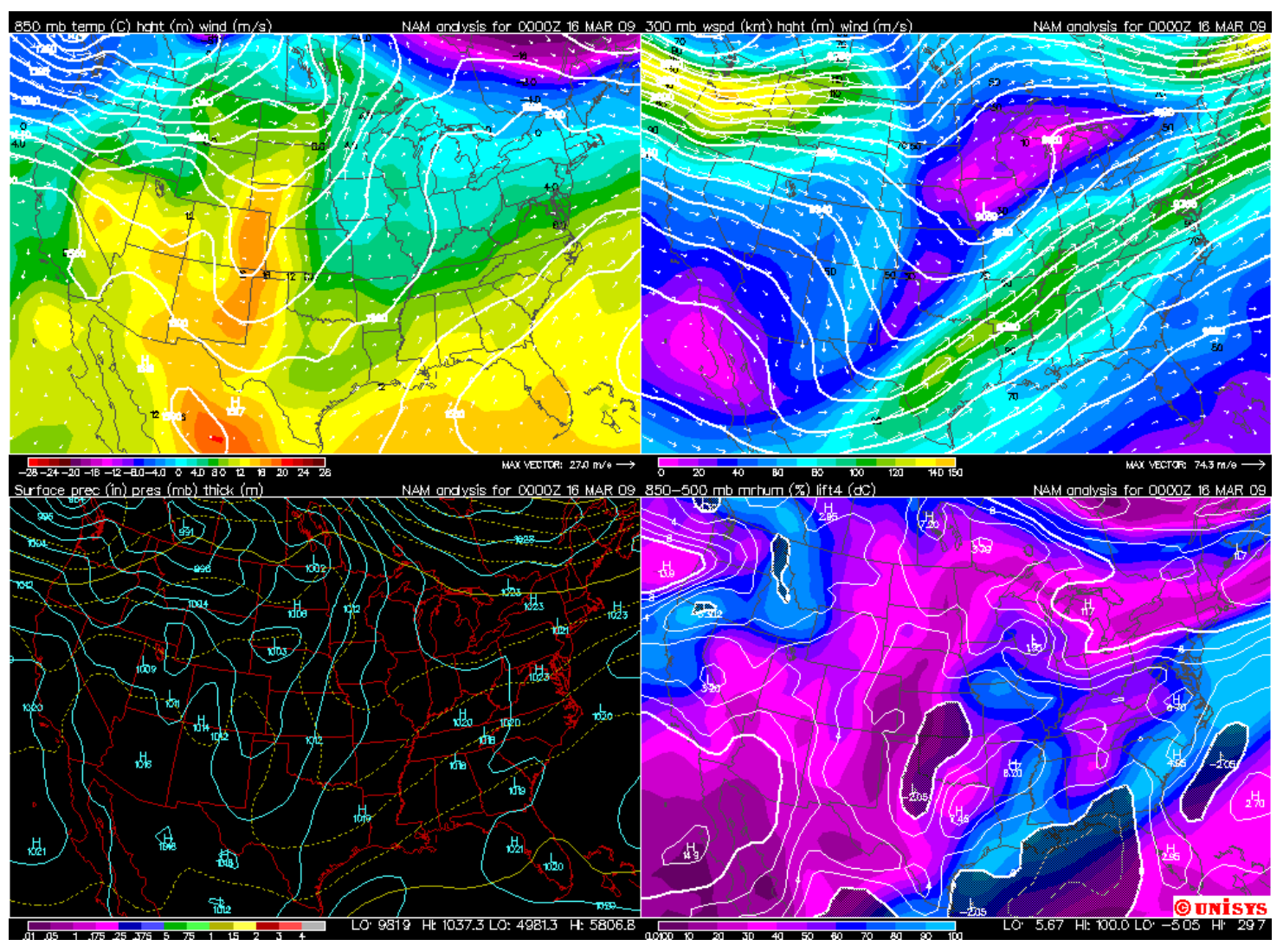

Fig 51: Example of synoptic conditions leading to a nowcasting situation in which the Persistence Method performs well, but the 700mb Wind and the Various Winds Methods perform poorly (Unisys 2010).

that clouds are neither created nor destroyed, if a cloud is not present in the initial Terra satellite pass, its location will not be predicted for comparison with the secondary Aqua satellite pass. As a result, the nowcasting methods perform poorly overall. An example of such a day can be seen in Figure (50).

The days in which the Various Winds Method (nine cases) or the 700mb Wind Method (five cases) performs poorly while the other two methods perform well do not possess as clear a signal as the previous two categories. There is evidence to suggest that upper-level troughs moving out of the region during the nowcasting period (thus 
returning it to straight west-east flow) tend to give these two displacement methods problems when predicting the movement of clouds. We speculate that as upper-level disturbances leave the Utah area, the west-east flow behind them not only moves the clouds out of the domain, but also dissipates a vast majority of them due to the lack of upper-level forcing. Again, any time clouds are generated or destroyed, the nowcasting methods will perform poorly. As to why the Persistence Method continues to perform well in these situations, it could be that it takes more time for lower clouds to be affected by the changing upper-level conditions in such a short timeframe. Since these clouds do not displace far due to their low-level in the atmosphere, the Persistence Method is still able to capture them. An example of such a day can be seen in Figure (51). 


\section{CONCLUSION}

\subsection{Research Conclusions}

This research has yielded several conclusions regarding cloud nowcasting techniques. We have presented several cloud nowcasting methods used to assess the location of clouds in a 0-6 hour timeframe. In order to properly evaluate these methods, we have created new skill evaluation techniques that appreciate both correct cloudy scenes and correct clear scenes.

Our hypothesis that clouds generated by terrain persist while others simply displace with the wind proved to only be partially true. While it is apparent from our results that the Persistence Method performed the best in terms of skill for all seasons but the summer, since the Terrain Methods incorporated elements from both the persistence and displacement methods, any departure from strict-persistence automatically resulted in lower skill scores. That's not to say that there were a few days where the Terrain Methods beat the Persistence method, but those were too few to draw any direct conclusions from.

Although trying to perform better than a persistence nowcast of cloud cover is difficult, it was surprising just how much better the Persistence Method did when compared to the other methods studied (by as much as $10 \%$ CSI during the winter months). This study was performed at quite a high resolution though ( $5 \mathrm{~km})$, so we speculate that studying real clouds in this high level of detail begs for more advanced displacement schemes than those performed here. 
Cloud phase information also proved invaluable in this study, showing that persisting the ice clouds while displacingeither the liquid water or mixed-phase clouds proved to be the most effective nowcasting techniques. Although this may seem to contradict our hypothesis of ice clouds simply moving with the wind, the Cloud Phase MODIS algorithm can only determine this information about the top of the cloud.

Thunderstorms, for example, include both ice cloud, mixed-phase cloud, and liquid water cloud. However, because they extend so high up in the atmosphere, MODIS would label them as ice cloud only. To assume that a cumulonimbus cloud (or any other type of deep cloud) that extends through the troposphere will move at the wind speed at its cloud top is unreasonable.

Maps of the domain depicting which methods work best in certain areas show exactly why the Persistence Method performed the best. Not only did it capture the stationary clouds over the terrain features, it also captured most of the clouds located everywhere else. These maps can be applied to create forecasts that combine nowcasting methods, such that one area of Utah can have the Persistence Method applied only, while another has the 700mb Wind Method applied to it.

In closing, a combination of the high-resolution cloud data used in this study, the presence of complex terrain, coupled with an inadequate cloud displacement scheme using real (but not current) wind data, and no sources and sinks of clouds, accounts for why the Persistence Method performs the best in our study over Utah. Although this was not the expected result, this research is still significant due to the speed of the methodology used. Time-sensitive military operations often need decisions made quickly, but cloud nowcast data assimilation models take a long time to run (on the order 
of an hour) and at much coarser resolution. This system can provide output in approximately two minutes or less, depending on the size of the area in question at $1 \mathrm{~km}$ resolution. Thus, the appropriate risk factors can be taken into account quickly to arrive at a final mission decision. 


\subsection{Research Limitations}

There are several limitations associated with this research that may lead to unforeseen problems with the conclusions drawn from these findings. Although our goal was to essentially create a simple nowcasting model using only actual observed data, this stipulation may have proven to be problematic.

The nature of the orbits of the Terra and Aqua satellites only allow for our domain to be fully sampled part of the time and not everyday. In addition, depending on the exact angle below each satellite, the resolutions of each MODIS sensor may not be exactly five kilometers. For example, if Terra passes quite close to Utah, the footprint it creates below it will essentially be the $5 \mathrm{~km}$ the Cloud Mask is said to possess. However, Aqua's orbit pattern is not the same as Terra's, and although it may also sample our entire domain, if the domain is significantly farther away from the satellite to either side, the resolution of the $5 \mathrm{~km}$ Cloud Mask may be coarser than the Terra pass.

Using real wind information is problematic because weather balloon soundings are only launched twice a day across the country. In many cases, wind information is essentially five hours old when it is applied to displacing the clouds in various directions. Depending on synoptic weather conditions (i.e. the passage of a front) these winds could have completely changed speed and direction by the time the nowcasting period arrived. In addition, assuming that the wind profile above Salt Lake City, UT applies to the entire state of Utah and southwest Wyoming may also be problematic.

Although this study aimed to use observational data only, the potential problems with the wind data suggest that perhaps this is not the best methodology to use. If gridded wind fields from various global weather models were utilized, perhaps this would 
improve the wind-driven nowcasting methods. Of course, utilizing this data depends upon the wind data availability and timing. That is, if acquiring the wind data requires a model to run for a significant amount of time, using that data is not practical. A second method to improve the wind data is to utilize satellite-derived winds, but disregarding the vertical location of the wind data itself. That is, simply using a nearest neighbor method to move the cloud at the closest wind speed data point and not worrying about the vertical placement of the data point or the cloud itself. This would avoid the issue our methodology encountered by the low vertical resolution of the satellite-derived winds over the domain.

The lack of moisture variables is also a hindrance since it prevents the creation or destruction of clouds. Using some sort of model-derived moisture field, clouds could be created if certain moisture thresholds are reached, or destroyed if it is displaced into a drier environment. Although, doing this does go against the initial goal of using observed data only.

The motion of the clouds themselves in the Various Winds Method could also be a problem. Each cloud top pressure and associated wind speed and direction assumes that the entire cloud (we assume it extends through the depth of the troposphere) will move at that speed. That is, once the cloud moves, there is only clear area left beneath it. Clouds are sheared apart on a regular basis, so to assume that the cloud-top pressure speed dictates the entire cloud's movement may not be accurate. 


\subsection{Future Work}

There are several possible routes in which to take this future work. The incorporation of a GOES Cloud Mask algorithm to fill in the gaps in-between Terra and Aqua satellite passes would be beneficial because once can evaluate the exact time at which a certain nowcasting method begins to fail. The incorporation of moisture variables in order to destroy and create clouds would be beneficial as well since these processes occur in nature quite often.

A combination of both GOES satellite-derived wind data and sounding data may prove to be more useful than the sounding data itself. Although the GOES wind data is at a coarser resolution, perhaps filling in the vertical gaps with sounding data may improve the cloud displacement methods. In addition, calculating a mean "cloud-motion" wind vector from multiple wind pressure levels instead of simply displacing them at their cloud-top pressure wind speed may improve the methods as well.

Additional cases and regions are beneficial to evaluate these nowcasting techniques over a longer term. Although not likely, it is possible that December 2008 to November 2009 simply proved to be an anomalous period over Utah for these case studies. 


\section{REFERENCES}

Ackerman, S. A., W. L. Smith, J. D. Spinhirne, and H. E. Revercomb, 1990: The 27-28 October 1986 FIRE IFO cirrus case study: spectral properties of cirrus clouds in

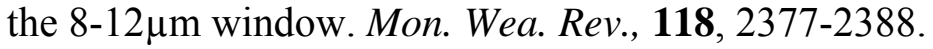

------, K. I. Strabala, W. P. Menzel, R. A. Frey, C. C. Moeller, L. E. Gumley, B. A. Baum, C. Schaaf, G. Riggs, 2010: Discriminating clear-sky from cloud with MODIS algorithm theoretical Basis document (MOD35) version 6.0. EOS ATBD website, $120 \mathrm{pp}$.

American Meteorological Society (AMS) Glossary, cited 2010: Nowcast. [Available online at http://amsglossary.allenpress.com/glossary].

Bradbury, D. L., and T. Fujita, 1968: Computation of height and velocity of clouds from dual, whole sky, time lapse picture sequences. Satellite and Mesometeorology Research Project Paper 70, 17 pp. [Available from Department of Geophysical Sciences, The University of Chicago, 5734 S. Ellis Ave., Chicago, IL 60637.]

Dills, P. N., and S. B. Smith, 1992: Comparison of profiler and satellite cloud tracked winds. Preprints, $6^{\text {th }}$ Conf. on Satellite Meteorology and Oceanography, Atlanta, GA, Amer. Meteor. Soc., 155-158.

Finley, J. P., 1884: Tornado Prediction. American Meteorological Journal, 1, 85-88.

Flueck, J. A., 1987: A study of some measures of forecast verification. Preprints, $10^{\text {th }}$ Conf. on Probability and Statistics in Atmospheric Sciences. Amer. Meteor. Soc., 69-73. 
Fujita, T. and Uchijima, 1963: Use of TIROS pictures for the internal structure of tropical storms. Mesoscale Research Project Paper 25, 25 pp. [Available from Department of Geophysical Sciences, The University of Chicago, 5734 S. Ellis Ave., Chicago, IL 60637.]

Gilbert, G. K. 1884: Finley's tornado predictions. American Meteorological Journal, 1, 166-172.

------, T. 1968: Present status of cloud velocity computations from ATS-1 and ATS-3. COSPAR Space Res., 9, 557-570.

Grose, A.M., E.A. Smith, H. Chung, M. Ou, B. Sohn and F.J. Turk, 2001: Possibilities and limitations for quantitative precipitation forecasts using nowcasting methods with infrared geosynchronous satellite imagery, Jour. Appl. Met., 41, 763-785.

Hall, Timothy J., R. N. Thessin, G. J. Bloy, and C. N. Mutchler, 2010: Analog ensemble scheme for objective short-term cloud forecasting. Preprints, $14^{\text {th }}$ Conf. on Aviation, Range, and Aerospace Meteorology, Atlanta, GA, Amer. Meteor. Soc., P195.

------, D. L. Reinke, and T. H. Vonder Haar, 1997: Forecasting applications of highresolution satellite cloud composite climatologies. Wea. Forecasting, 13, 16-23.

Hamill, T. M., and T. Nehrkorn, 1993: A short-term cloud forecast scheme using cross correlations. Wea. Forecasting, 8, 401-411.

Joyce, R. J., J. E. Janowiak, P. A. Arkin, and P. Xie, 2004: CMORPH: A method that produces global precipitation estimates from passive microwave and infrared data at high spatial and temporal resolution. J. Hydrometeor., 5, 487-503. 
Kopp, T. J., M. M. Wonsick, L. E. Cantrell, and F. D. Bieker, 1997: ADVCLD: Air Force Global Weather Center's updated cloud forecast model. Preprints, Cloud Impacts on DOD Operations and Systems Conf., Newport, RI, Department of Defense, 107-110. [NTIS ADA 330020].

Kidder, S. Q., and T. H. Vonder Haar, 1995: Satellite Meteorology: An Introduction. Academic Press, 456 pp.

Leese, J. A., and C. S. Novak, 1971: An automated technique for obtaining cloud motion from geosynchronous satellite data using cross correlation. J. Appl. Meteor., 16, 118-132.

Menzel, William P., 2001: Cloud tracking with satellite imagery: From the pioneering work of Ted Fujita to the present. Bull. Amer. Meteor. Soc., 82, 33-47.

------, B. A. Baum, K. I. Strabala, and R. A. Frey, 2006: Cloud top properties and cloud phase ATBD version 6. EOS ATBD website, $56 \mathrm{pp}$.

------, R. A. Frey, H. Zhang, D. P. Wylie, C. C. Moeller, R. E. Holz, B. Maddux, B. A. Baum, K. I. Strabala, and L. E. Gumley, 2007: MODIS global cloud-top pressure and amount estimation: Algorithm description and results. J. Appl. Meteor., 47, $1175-1198$.

Modica, G. D., R. d'Entremont, E. Mlawer, and E. Gustafson, 2010: Short-range solar radiation forecasts in support of smart grid technology. Preprints, $1^{\text {st }}$ Conf. on Weather, Climate, and the New Energy Economy, Atlanta, GA, Amer. Meteor. Soc., J12.3

Norquist, Donald C. 2000: Cloud Predictions Diagnosed from Global Weather Model Forecasts. Mon. Wea. Rev., 128, 3538-3555. 
Reinke, D. L., C. L. Combs, E. M. Tomlinson, and T. H. Vonder Haar, 1990: Persistence forecasts from high-resolution cloud composite climatologies. Proc. 1990 Cloud Impacts on DoD Systems Conf., Monterey, CA, U.S. Navy, 145-147.

Sharman, B., 2010: Turbulence forecasts for manned and unmanned aerial vehicles. $7^{\text {th }}$ Conf. on Battlespace Atmospheric and Cloud Impacts on Military Operations., Omaha, NE, P2.3.

Shirkey, R., 2010: Army research lab tactical decision aides and related work. $7^{\text {th }}$ Conf. on Battlespace Atmospheric and Cloud Impacts on Military Operations., Omaha, NE, P6.1.

Soden, B. J., and F. P. Bretherton, 1993: Upper tropospheric relative humidity from the GOES $6.7 \mu \mathrm{m}$ channel: Method and climatology for July 1987. J. Geophys. Res., 98, 16669-16688.

Strabala, K. I., S. A. Ackerman, and W. P. Menzel, 1993: Cloud properties inferred from 8 - 12 - $\mu$ m data. J. Appl. Meteoro., 33, 212-229.

The International Satellite Cloud Climatology Project (ISCCP), cited 2010: What is the ISCCP? [Available online at http://isccp.giss.nasa.gov].

Unisys Weather, cited 2010. [Avalable online at http://weather.unisys.com/archive/].

Williams, M., and R. A. Houze, 1987: Satellite-observed characteristics of winter monsoon cloud clusters. Mon. Wea. Rev., 115, 505-519.

Wilks, Daniel S., 2006: Statistical methods in the atmospheric sciences. Academic Press, $630 \mathrm{pp}$. 